\title{
TRANSGRESSIONS OF THE EULER CLASS AND EISENSTEIN COHOMOLOGY OF $\mathrm{GL}_{N}(\mathrm{Z})$
}

\author{
NICOLAS BERGERON, PIERRE CHAROLLOIS, AND LUIS E. GARCIA
}

\begin{abstract}
These notes were written to be distributed to the audience of the first author's Takagi lectures delivered June 23, 2018. These are based on a work-in-progress that is part of a collaborative project that also involves Akshay Venkatesh.

In this work-in-progress we give a new construction of some Eisenstein classes for $\mathrm{GL}_{N}(\mathbf{Z})$ that were first considered by Nori [41] and Sczech [44]. The starting point of this construction is a theorem of Sullivan on the vanishing of the Euler class of $\mathrm{SL}_{N}(\mathbf{Z})$ vector bundles and the explicit transgression of this Euler class by Bismut and Cheeger. Their proof indeed produces a universal form that can be thought of as a kernel for a regularized theta lift for the reductive dual pair $\left(\mathrm{GL}_{N}, \mathrm{GL}_{1}\right)$. This suggests looking to reductive dual pairs $\left(\mathrm{GL}_{N}, \mathrm{GL}_{k}\right)$ with $k \geq 1$ for possible generalizations of the Eisenstein cocycle. This leads to fascinating lifts that relate the geometry/topology world of real arithmetic locally symmetric spaces to the arithmetic world of modular forms.

In these notes we don't deal with the most general cases and put a lot of emphasis on various examples that are often classical.
\end{abstract}

\section{CONTENTS}

1. Introduction

2. Thom and Euler classes and torus bundles $\quad 7$

3. The Eisenstein class 9

4. The universal space of oriented quadratic spaces 12

5. Mathai-Quillen Thom form on the universal space of oriented quadratic spaces 14

6. Some explicit formulas for the Mathai-Quillen Thom form 18

7. Canonical transgression of the Thom form 21

8. Eisenstein transgression 24

9. The Eisenstein transgression for $\mathrm{GL}_{2}(\mathbf{R})$ and classical modular forms 28

10. Relation between the Eisenstein transgression and the Eisenstein class 31

11. More $\mathrm{SL}_{2}(\mathbf{R})$ computations : Dedekind-Rademacher and Damerell results 33

12. Adelic formulation, period computations and the Klingen-Siegel Theorem 36

13. Eisenstein theta correspondence for the dual pair $\left(\mathrm{GL}_{a}, \mathrm{GL}_{b}\right) \quad 43$

$\begin{array}{ll}\text { References } & 50\end{array}$

\section{INTRODUCTION}

These notes are based on the Takagi lectures that were delivered June 23, 2018, by the first author. The aim of these lectures was to tell a story that starts with the topology of $\mathrm{SL}_{N}(\mathbf{Z})$ vector bundles and abuts to a natural mechanism for constructing mappings from the homology of congruence subgroups of $\mathrm{GL}_{N}(\mathbf{Z})$ to the arithmetic world of modular forms. This mechanism is a certain 'regularized $\theta$-lift.' 
The full story is still a work-in-progress that is part of a collaborative project that also involves Akshay Venkatesh. The current notes put emphasis on the most classical aspects of the story. These reflect the lectures quite faithfully, in particular we have tried to maintain the spirit of 'story telling.' There are only few complete formulations of new results. Complete statements and detailed proofs will appear in forthcoming paper(s). Our primary hope is that the present notes will serve to show that the 'regularized $\theta$-lift' we consider is interesting in itself.

In the following of this introduction, we outline the whole story in order to help the reader going through the whole notes.

1.1. Topology. In a short note [49] in 1975, Sullivan proved:

Theorem 1. Let $M$ be a connected oriented manifold. The Euler class of an oriented $\mathrm{SL}_{N}(\mathbf{Z})$ vector bundle on $M$ vanishes rationally.

This answered a question of Milnor. Sullivan's proof is beautiful and amazingly short: an oriented $\mathrm{SL}_{N}(\mathbf{Z})$ vector bundle on $M$ yields a group bundle $T \rightarrow M$ whose fibers are $N$-dimensional tori $\mathbf{R}^{N} / \mathbf{Z}^{N}$. Denote by $\{0\} \subset T$ the image of the zero section. Given a fixed positive integer $m$, Sullivan introduces the submanifold $T[m] \subset T$ that consists of $m$-torsion points. The key observation of the proof is then that a nonzero multiple of $T[m]-m^{N}\{0\}$ is a boundary in $T$. In other words, the rational homology class of $T[m]-m^{N}\{0\}$ is zero. We give a detailed cohomological proof of this in Section 2 and explain why this implies Theorem 1.

This does not mean that the homology class $\left[T[m]-m^{N}\{0\}\right]$ is uninteresting. Quite the contrary in fact: with respect to the torsion linking form it is dual to an interesting cohomology class in $H^{N-1}(T-T[m], \mathbf{Q} / \mathbf{Z})$. In Section 3 we refine Sullivan's observation and prove:

Theorem 2. Let $m$ be a positive integer. There exists a distinguished preimage

$$
z_{m} \in H^{N-1}(T-T[m], \mathbf{Q})
$$

of $\left[T[m]-m^{N}\{0\}\right]$.

Moreover: the class $z_{m}$ is in fact 'almost integral,' see Definition 10 and the Remark following it.

The construction is similar to Faltings's construction [23] of the Eisenstein symbol on Siegel spaces.

1.2. Geometry. Bismut and Cheeger [8] have refined Theorem 1 by constructing an explicit primitive of the Chern-Weil differential form representing the Euler class. Building on their work, we construct a smooth differential form $E_{\psi}$ of degree $N-1$ on $T-\{0\}$ such that the following theorem holds.

Theorem 3. Let $m$ be a positive integer. The expression

$$
E_{\psi}^{(m)}:=m^{*} E_{\psi}-m^{N} E_{\psi}
$$

defines a closed differential form of degree $N-1$ on $T-T[m]$ whose cohomology class in $H^{N-1}(T-T[m], \mathbf{R})$ is equal to the class $z_{m}$ of Theorem 2.

Here the notation $E_{\psi}$ refers to the fact that this differential form is constructed as an Eisenstein series, and $m: T \rightarrow T$ denotes the map 'multiplication by $m$ ' in the fibers.

In these notes we only deal with the particular situation where $T$ is the total space of a universal family of metrized real tori over a locally symmetric space $M=\Gamma \backslash X$, where 
$X=X_{N}$ is the symmetric space associated to $\mathrm{SL}_{N}(\mathbf{R})$ and $\Gamma$ is a congruence subgroup of $\mathrm{GL}_{N}(\mathbf{Z})$.

The construction of the Eisenstein series $E_{\psi}$ relies on the work of Mathai and Quillen [39] that we discuss in Sections 4 and 5. It consists in the construction of a canonical (Gaussian) differential form representing the Thom class of a metrized oriented vector bundle, and in the construction of a canonical primitive - or transgression - of its pullback to the sphere bundle. This part is purely local (Archimedean). Working $\mathrm{GL}_{N}(\mathbf{R})$-equivariantly the Gaussian Thom form can be represented by a cocycle in the $\left(\mathfrak{g l}_{N}(\mathbf{R}), \mathrm{SO}_{N}\right)$-complex of the representation of $\mathrm{GL}_{N}(\mathbf{R})$ in the space of Schwartz functions on $\mathbf{R}^{N}$. This cocycle behaves in several ways like the cocycle constructed by Kudla and Millson in [38], and the construction of the transgression form produces a $(N-1)$ form that behaves like their form $\psi$; that both cocycles should be regarded as analogous follows from previous work of the third author [25], which contains an approach to Kudla and Millson's results using Mathai and Quillen's ideas. We provide explicit formulas for all these forms in Sections 6 and 7.

In Section 8 we apply the theta machinery to these special Schwartz forms. The result is precisely the Eisenstein transgression form $E_{\psi}$.

Let $v \in \mathbf{R}^{N} / \mathbf{Z}^{N}$ be a nonzero torsion point that is fixed by $\Gamma$. To $v$ corresponds a section $M \rightarrow T-\{0\}$. Pulling back $E_{\psi}$ by this section gives a closed $(N-1)$-form $E_{\psi, v}$ on $M$ that represents an Eisenstein class in $H^{N-1}(\Gamma, \mathbf{Q}) .{ }^{1}$ Similar classes were first, and almost simultaneously, considered by Nori [41] and Sczech [43, 44] in the beginning of the 90 's. We will clarify the exact relations between these classes in our forthcoming joint paper.

Pulling back $E_{\psi}$ by the zero section gives the Bismut-Cheeger transgression of the Euler form. It plays a key role in their proof of the Hirzebruch conjecture on the signature of the Hilbert modular varieties (first proved by Atiyah, Donnelly and Singer [1] and Müller [40]).

Here we insist to consider the Eisenstein transgression form $E_{\psi}$ on the total bundle. In Section 9 we compute $E_{\psi}$ in the $N=2$ case; it is the real part of an 'almost holomorphic' 1 -form on the universal elliptic curve. In fact this 1-form decomposes as a sum of two forms (see (9.6)): the first is the pull-back to the universal elliptic curve of

$$
E_{2}(\tau, z) d \tau,
$$

where $E_{2}$ is the standard weight two Eisenstein series, and the restriction of the second to a fiber is given by

$$
E_{1}(\tau, z) d z
$$

where $E_{1}$ is the standard weight one Eisenstein series.

We sketch a proof of Theorem 3 in Section 10. In Section 11 we come back to the case $N=2$ and relate the forms $E_{\psi}^{(m)}$ and $E_{\psi, v}$ to classical modular functions.

1.3. First applications to number theory. At the end of Section 11 we give a 'topological' proof of a classical theorem of Damerell [20] on the algebraicity of the evaluation of weight one Eisenstein series at CM points. To do so we restrict the form $E_{\psi}^{(m)}$ to a fixed $\mathrm{CM}$ elliptic curve in the fiber and use that it has rational periods by Theorem 3 .

\footnotetext{
${ }^{1}$ Pulling back $E_{\psi}$ by the zero section gives the Bismut-Cheeger transgression of the Euler form.
} 
Damerell's theorem is related to the algebraicity of some special values of Hecke $L$ functions associated to imaginary quadratic fields. Long before that, in 1735, Euler computed the values of the Riemann zeta function at even positive integers:

$$
\zeta(2 k)=\sum_{n=1}^{+\infty} \frac{1}{n^{2 k}}=(-1)^{k+1} \frac{(2 \pi)^{2 k}}{2(2 k) !} B_{2 k}
$$

where $B_{m}$ is the $m$-th Bernoulli number - a rational number. Using the functional equation, Euler's formula can be rephrased as the evaluation of the Riemann zeta function at non-positive integers:

$$
\zeta(-k)=(-1)^{k} \frac{B_{k+1}}{k+1} \text { for all } k \geq 0 .
$$

In 1924 Hecke observed that zeta functions of real quadratic fields take rational values at non-positive integers, and he suggested a method of proof based on the Fourier expansion of Hilbert modular forms. Siegel [46] gave the first full proof for arbitrary totally real number fields in 1937, using the theory of quadratic forms instead. In 1962 Klingen [36] completed and extended Hecke's program to arbitrary totally real number fields, and later Shintani [45] and many others published different proofs or extensions of this theorem that is now usually referred to as the 'Klingen-Siegel Theorem.' In the survey [33], Ishii and Oda give a beautiful account of this rich story. ${ }^{2}$

The signature conjecture of Hirzebruch was the first hint that certain zeta values have a topological interpretation. In the 1990's Nori and Sczech have introduced their abovementioned cocycles in order to investigate topologically all special values of zeta and $L$ functions of totally real number fields. As a direct consequence of the rationality of their Eisenstein classes they recover the Klingen-Siegel Theorem.

In Section 12 we show how our methods yield to another natural proof of Hecke's conjecture. Working adelically, it is indeed pretty straightforward to compute the integral of the closed $(N-1)$-form $E_{\psi, v}$ along the $(N-1)$-dimensional submanifold of $M$ associated to the group of units of a degree $N$ totally real number field $F$, see Theorem 25 . It is essentially equal to the value at $s=0$ of the zeta function of $F$.

Now Theorem 3 implies that this value is rational, and even 'almost integral.' The rationality theorem of Klingen and Siegel therefore immediately follows. In fact we recover the following integrality theorem for zeta values of totally real number fields at non-positive integers: let $\mathfrak{f}$ and $\mathfrak{b}$ be two relatively prime ideals in the ring of integers $\mathcal{O}_{F}$. The partial zeta function associated to the ray class $\mathfrak{b} \bmod \mathfrak{f}$ is defined by

$$
\zeta(\mathfrak{b}, \mathfrak{f}, s):=\sum_{\mathfrak{a} \equiv \mathfrak{b} \bmod \mathfrak{f}} \frac{1}{N(\mathfrak{a})^{s}}, \quad \operatorname{Re}(s)>1,
$$

where $\mathfrak{a}$ runs over all integral ideals in $\mathcal{O}_{F}$ such that the fractional ideal $\mathfrak{a b}^{-1}$ is a principal ideal generated by a totally positive number in the coset $1+\mathfrak{f b}^{-1}$.

Theorem 4 (Deligne-Ribet, Cassou-Noguès [22, 14]). Let $\mathfrak{c}$ be an integral ideal coprime to $\mathfrak{f b}^{-1}$. Then we have:

$$
n(\mathfrak{c}) \zeta(\mathfrak{b}, \mathfrak{f}, 0)-\zeta(\mathfrak{b} \mathfrak{c}, \mathfrak{f}, 0) \in \mathbf{Z}\left[\frac{1}{n(\mathfrak{c})}\right] .
$$

Here $n$ denotes the norm.

\footnotetext{
${ }^{2}$ As Serre pointed out to us, an interested reader should also take a look at [48, p. 101].
} 
We only deal with the value at $s=0$ in these notes, but considering Eisenstein cohomology classes with local coefficients allows one to deal with all negative integers.

In $[15,16]$ Charollois, Dasgupta and Greenberg have defined an integral versions of Sczech's cocycle, and recently, considering as above the cohomology of $T-T[m]$ but using the so called 'logarithm sheaf' rather that $\mathbf{Q}$ as coefficients, Beilinson, Kings and Levin [2] have developed a 'topological polylogarithm' that provides an integral version of Nori's Eisenstein classes. As a consequence both these works give new proofs of Theorem 4. Beside giving an interpretation of the numbers occurring in Theorem 4 as linking numbers, one novel aspect of our viewpoint is that we produce canonical closed invariant differential forms. These naturally lead to consider invariant cohomology and give rise to arithmetic $\theta$-lifts that will be explored in detail in our forthcoming joint work with Venkatesh. We only briefly allude to them in the next paragraph.

Finally, since our methods allow to deal with both Damerell's Theorem - on Hecke $L$ functions of imaginary quadratic fields - and the Klingen-Siegel Theorem — on Hecke $L$ functions of totally real number fields - it is natural to wonder if these shed some light on $L$-functions associated to general characters. For these $L$-functions the vast conjectures of Deligne [21] have been verified in many cases - see in particular Blasius [9] and Colmez [19] — and have been announced by Harder [29]. In turns out that our viewpoint yields to a direct proof of this theorem and furthermore allows to deal with integrality features. We briefly allude to it at the end of Section 13 and will provide details in a forthcoming paper.

1.4. An Eisenstein $\theta$-lift. The (left) linear action of $\mathrm{GL}_{N}$ on column vectors and the (right) action of $\mathrm{GL}_{1}$ by scalar multiplication turn $\left(\mathrm{GL}_{N}, \mathrm{GL}_{1}\right)$ into a 'dual pair' in the sense of Howe [32]. To be more precise, it is a particular example of an irreducible reductive dual pair of type II. In general such a pair is a couple of linear groups $\left(\mathrm{GL}_{a}, \mathrm{GL}_{b}\right)$ embedded in $\mathrm{GL}_{a b}$ seen as the linear group of the space of $a \times b$ matrices. Then $\mathrm{GL}_{a}$ and $\mathrm{GL}_{b}$ commute in $\mathrm{GL}_{a b}$ and one can prove that there is a natural correspondence - the $\theta$-correspondence - between automorphic forms of $\mathrm{GL}_{a}$ and $\mathrm{GL}_{b}$.

It turns out that one can think of the closed differential form $E_{\psi}$ as a regularized theta lift of the trivial character, for the dual pair $\left(\mathrm{GL}_{N}, \mathrm{GL}_{1}\right)$. This suggests looking more generally at dual pairs $\left(\mathrm{GL}_{a}, \mathrm{GL}_{b}\right)$. In these notes we focus on the case $a=N, b=2$.

The topological picture is the following: consider a modular curve $Y$ (maybe with level structure) and let $\mathcal{E} \rightarrow Y$ be the corresponding universal family of elliptic curves over $Y$. Let $\Gamma$ be a congruence subgroup in $\mathrm{GL}_{N}(\mathbf{Z})$. The group $\Gamma$ acts in the natural way on the $N$-fold fiber product $\mathcal{E}^{N}$ over $Y$. The latter is a subfamily of metrized $2 N$-dimensional real tori and, fixing a positive integer $m$, the restriction of the $(2 N-1)$-class $z_{m}$ to this family can be realised as a $\Gamma$-equivariant class

$$
\Theta_{m} \in H_{\Gamma}^{2 N-1}\left(\mathcal{E}^{N}-\mathcal{E}^{N}[m]\right) .
$$

A de Rham representative is indeed obtained by restricting the closed $(2 N-1)$-form $E_{\psi}^{(m)}$ to

$$
\Gamma \backslash\left(X \times\left(\mathcal{E}^{N}-\mathcal{E}^{N}[m]\right)\right) .
$$

In our forthcoming work we associate to the class (1.4) a group cohomology class in $H^{N-1}(\Gamma, \mathcal{M})$, where $\mathcal{M}$ is a $\Gamma$-space of degree $N$ meromorphic forms on $\mathcal{E}^{N}$ that are holomorphic on a complement of finitely many elliptic hyperplanes. Evaluating the elements of $\mathcal{M}$ on $\Gamma$-invariant torsion sections of $\mathcal{E}^{N}$, we get: 
Theorem 5. Let $m$ be a positive integer. To any $\Gamma$-invariant nowhere zero torsion section $x: Y \rightarrow \mathcal{E}^{N}$ of order prime to $m$ corresponds a class

$$
x^{*}\left(\Theta_{m}\right) \in H^{N-1}\left(\Gamma, M_{N}(Y)\right),
$$

where $M_{N}(Y)$ denotes the space of weight $N$ modular forms on $Y$ endowed with the trivial T-action.

One outcome of this theorem is the existence of fascinating and explicit homomorphisms

$$
H_{N-1}(\Gamma, \mathbf{Z}) \rightarrow M_{N}(Y)
$$

that relate the geometry/topology world of real arithmetic quotient of $X_{N}$ to the arithmetic world of modular forms. The existence of such maps was first discovered by one of us (P.C.) by quite different methods, see [17].

Example. Consider the case $N=2$ with $Y=Y_{1}(\ell)$ and $\ell>1$. It was already observed by Borisov and Gunnells $[10,11]$ that products

$$
E_{a / \ell}^{(1)} E_{b / \ell}^{(1)}, \quad a, b=1, \ldots, \ell-1,
$$

of weight one modular forms

$$
E_{a / \ell}^{(1)}:=E_{1}(\tau, a / \ell)
$$

satisfy, modulo Eisenstein series, the same relations as Manin symbols in the homology of

$$
\Gamma=\Gamma_{1}(\ell):=\left\{\left(\begin{array}{ll}
a & b \\
c & d
\end{array}\right) \in \mathrm{SL}_{2}(\mathbf{Z}):\left(\begin{array}{ll}
a & b \\
c & d
\end{array}\right) \equiv\left(\begin{array}{ll}
1 & * \\
0 & 1
\end{array}\right)(\bmod \ell)\right\} .
$$

This is in fact related to the existence of a homomorphism $H_{1}\left(\Gamma_{1}(\ell), \mathbf{Z}\right) \rightarrow M_{2}\left(Y_{1}(\ell)\right)$ as discussed above in greater generality.

In these notes we restrict our discussion to a de Rham realisation of the lifts (1.6). The restriction of $E_{\psi}^{(m)}$ to (1.5) defines a kernel. In Section 13 we explain how this kernel is a particular instance of a regularized theta lift and gives rise to mappings (1.6). We will relate these to the classes of Theorem 5 in our forthcoming work. Here we explain how the theta lift construction allows us to evaluate the morphisms (1.6) along tori: let $U \subset \Gamma$ be a subgroup of maximal rank $N-1$ of positive integral units in a totally real number field $F$ of degree $N$ over $\mathbf{Q}$ (and embedded in $\Gamma$ via a regular representation). In the terminology of Kudla [37] we have a seesaw of dual pairs in $\mathrm{GL}_{2 N}(\mathbf{Q})$ :

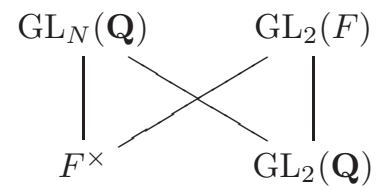

and it yields the following:

Theorem 6. The evaluation of a map (1.6) on the image in $H_{N-1}(\Gamma, \mathbf{Z})$ of the fundamental class in $H_{N-1}(U, \mathbf{Z})$ is a modular form of weight $N$ obtained as the restriction to the diagonal $\mathcal{H} \subset \mathcal{H}^{N}$ of a Hecke-Eisenstein modular form of weight $(1, \ldots, 1)$ for a congruence subgroup of $\mathrm{PGL}_{2}\left(\mathcal{O}_{F}\right)$.

The morphisms (1.6) govern surprising relations between some modular forms. But this is only the shadow of a richer story that also involves relations between modular units considered by Beilinson and Kato [5, 35]. We conclude these notes by addressing this question after composition by regulators. 
Consider for instance the ring $\mathcal{O}\left(Y_{1}(\ell)\right)$ of holomorphic functions on the modular curve $Y_{1}(\ell)$ for some integer $\ell>1$. The map

$$
\log |\cdot|: \mathcal{O}\left(Y_{1}(\ell)\right)^{\times} \rightarrow A^{0}\left(Y_{1}(\ell)\right)
$$

is an example of a regulator in degree 1. A more interesting example, in degree 2, is induced by the map

$$
\begin{aligned}
\operatorname{reg}: \wedge^{2} \mathcal{O}\left(Y_{1}(\ell)\right)^{\times} & \rightarrow A^{1}\left(Y_{1}(\ell)\right) \\
u \wedge u^{\prime} & \mapsto i \log \left|u^{\prime}\right| d \arg u-i \log |u| d \arg u^{\prime} .
\end{aligned}
$$

In the last paragraph of Section 13 we briefly explain how our regularized theta lift for the dual pair $\left(\mathrm{GL}_{2}, \mathrm{GL}_{2}\right)$ can be interpreted as defining a 1-form on $Y(\ell)$ for every pair of cusps in $\mathbf{P}^{1}(\mathbf{Q})$; moreover, these 1-forms are obtained as the regulator of a product of two modular units. This fits with the work of Brunault [13] on the explicit Beilinson-Kato relations.

1.5. Acknowledgments. N.B. would like to thank the Mathematical Society of Japan, the local organisers, and especially Professor Kobayashi, for their invitation and their kind hospitality in Kyoto. We all thank our collaborator Akshay Venkatesh as well as Javier Fresan for their comments and corrections on these notes. L.G. wishes to thank IHES for providing excellent conditions for research while this work was done. L.G. also acknowledges financial support from the ERC AAMOT Advanced Grant.

\section{THOM AND EULER CLASSES AND TORUS BUNDLES}

In this section we first define the Thom and Euler classes of an oriented vector bundle. Then we explain Sullivan's proof of Theorem 1 in terms that set the stage for the Eisenstein class that we will introduce in the next section.

2.1. Thom and Euler classes of oriented vector bundles. Let $M$ be a closed connected oriented $d$-manifold and let $E$ be an oriented, real vector bundle of rank $N \geq 2$ over $M$. We shall denote by $E_{0}$ the image of the zero section $\sigma_{0}: M \rightarrow E$ that embeds $M$ into $E$.

The Thom isomorphism identifies the cohomology of $E$ with compact support in the vertical direction ${ }^{3}$

$$
H^{\bullet}\left(E, E-E_{0}\right)
$$

with $H^{\bullet-N}(M)$, the cohomology of the base shifted by $N$.

Definition 7. Under the isomorphism

$$
H^{\bullet}(M) \stackrel{\sim}{\rightarrow} H^{\bullet+N}\left(E, E-E_{0}\right)
$$

the image of 1 in $H^{0}(M)$ determines a cohomology class in $H^{N}\left(E, E-E_{0}\right)$, called the Thom class of the oriented vector bundle $E$.

Remark. It will be important for us that, since $M$ is oriented, the Thom class of $E$ and the Poincaré dual of the zero section of $E$ can be represented in de Rham cohomology by the same differential form (see [12, Proposition 6.24]).

\footnotetext{
${ }^{3}$ Unless otherwise explicitly specified all (co-)homology groups are with integral coefficients.
} 
Definition 8. The pullback of the Thom class to $M$ by the zero section $\sigma_{0}: M \rightarrow E$ determines a cohomology class

$$
e(E) \in H^{N}(M)
$$

called the Euler class.

Remark. If $M$ is smooth, the Euler class measures the obstruction to the existence of a nowhere vanishing section: let $s: M \rightarrow E$ be a generic smooth section and let $Z \subset M$ be its zero locus. Then $Z$ represents a homology class $[Z] \in H_{d-N}(M)$, and $e(E)$ is the Poincaré dual of $[Z]$.

2.2. An observation of Sullivan. We now furthermore assume that the structure group $\Gamma$ of $E$ can be reduced to $\mathrm{SL}_{N}(\mathbf{Z})$, or equivalently that the vector bundle $E$ contains a subbundle $E_{\mathbf{Z}}$ whose fibers are lattices isomorphic to $\mathbf{Z}^{N}$. Denote by $T$ the quotient bundle $E / E_{\mathbf{Z}}$; this is a group bundle of base $M$, whose fibers are $N$-dimensional tori $\mathbf{R}^{N} / \mathbf{Z}^{N}$.

The zero section of $E$ projects onto a section $0: M \rightarrow T$ whose image we denote by $\{0\}$. Now let $m$ be a positive integer. We denote by $T[m]$ the submanifold of $T$ that consists of $m$ torsion points.

The following lemma is due to Sullivan [49]:

Lemma 9. We have:

$$
\left[T[m]-m^{N}\{0\}\right]=0 \quad \text { in } H^{N}(T, \mathbf{Q}) .
$$

Proof. Let us denote by $m$ the finite cover $T \rightarrow T$ given by multiplication by $m$ in each fiber. To $m$ correspond two maps, the direct and inverse image maps, in cohomology:

$$
m_{*}: H^{\bullet}(T) \rightarrow H^{\bullet}(T) \text { and } m^{*}: H^{\bullet}(T) \rightarrow H^{\bullet}(T) .
$$

Since $m$ is a covering map of degree $m^{N}$, we have

$$
m_{*} m^{*}=m^{N} \text { on } H^{\bullet}(T) .
$$

Now $m^{*}[\{0\}]=[T[m]]$ in $H^{N}(T)$ and $m_{*}[\{0\}]=[\{0\}]$. It follows that

$$
m_{*}\left(\left[T[m]-m^{N}\{0\}\right]\right) \text { vanishes in } H^{N}(T) .
$$

By (2.1) the map $m_{*}$ is injective over the rationals, and the lemma follows.

Remark. It follows from the proof that for any integer $\ell$ coprime to $m$ the cohomology class $\left[T[m]-m^{N}\{0\}\right]$ vanishes in $H^{N}\left(T, \mathbf{Z}_{\ell}\right)$, or equivalently that $\left[T[m]-m^{N}\{0\}\right]$ vanishes in $H^{N}(T, \mathbf{Z}[1 / m])$.

2.3. Vanishing of the rational Euler class. Let us now explain why Lemma 9 implies that the rational Euler class of the normal bundle of $\{0\}$ in $T$ vanishes.

First observe that under the map

$$
H^{N}(T, T-\{0\}) \rightarrow H^{N}(T)
$$

the Thom class is mapped onto the class $[\{0\}]$ dual to the zero section in $T$.

Now, since $m \cdot 0=0$, we have $0^{*} m^{*}=0^{*}$ and therefore

$$
0^{*}(T[m])=0^{*}([\{0\}]) \in H^{N}(M) .
$$

We finally conclude from (2.2) and (2.3) that

$$
\left(1-m^{N}\right) 0^{*}([\{0\}])=0^{*}\left(T[m]-m^{N}\{0\}\right) \text { vanishes in } H^{N}(M, \mathbf{Q}) .
$$


In particular the rational Euler class $0^{*}([\{0\}]) \in H^{N}(M, \mathbf{Q})$ of the normal bundle of $\{0\}$ in $T$ vanishes.

The normal bundle of $\{0\}$ in $T$ being isomorphic to $E$ this finally forces $e(E)$ to be a torsion class and Theorem 1 is proved.

Remark. The proof shows that the order of $e(E)$ in $H^{N}(M, \mathbf{Z})$ is a divisor of the g.c.d. of the integers $m^{N}\left(m^{N}-1\right)$ as $m$ varies. This g.c.d. is the denominator of $\frac{1}{2} B_{N}$ where $B_{N}$ is the $N$-th Bernoulli number, see [31, Theorem 118].

\section{THE EISENSTEIN ClASS}

Sullivan's observation - Lemma 9 above - does not imply that the homology class $\left[T[m]-m^{N}\{0\}\right]$ is uninteresting. Quite the contrary in fact: computing linking number with $T[m]-m^{N}\{0\}$ indeed produces interesting cohomology classes. In this section we explain how to extract a canonical class from this. Let first fix a positive integer $m>1$.

3.1. Linking with $T[m]-m^{N}\{0\}$. Consider the long exact sequence in cohomology associated to the pair $(T, T[m])$ :

$$
\cdots \rightarrow H^{N-1}(T) \rightarrow H^{N-1}(T-T[m]) \rightarrow H^{N}(T, T-T[m]) \rightarrow H^{N}(T) \rightarrow \cdots
$$

The Thom isomorphism induces

$$
H^{N}(T, T-T[m]) \stackrel{\sim}{\longrightarrow} H^{0}(T[m]) .
$$

The class in $H^{N}(T, T-T[m])$ corresponding to $\left[T[m]-m^{N}\{0\}\right] \in H^{0}(T[m])$ has a trivial image in $H^{N}(T, \mathbf{Q})$ by Lemma 9. It follows that it can be lifted as a class in $H^{N-1}(T-T[m], \mathbf{Q})$, but such a lift is only defined up to $H^{N-1}(T, \mathbf{Q})$.

In the next paragraph we explain how to pick a canonical lift. To do so we follow a refinement, due to Faltings [23], of Sullivan's observation.

Remarks. 1. We may think of $H^{0}(T[m])$ as $\Gamma$-invariant 'divisors' — or rather formal linear combinations - of $m$-torsion points in the $N$-torus $\mathbf{R}^{N} / \mathbf{Z}^{N}$. The covering map $m: T \rightarrow T$ induces a (direct image) map $H^{N}(T, T-T[m]) \rightarrow H^{N}(T, T-\{0\})$ such that the following diagram

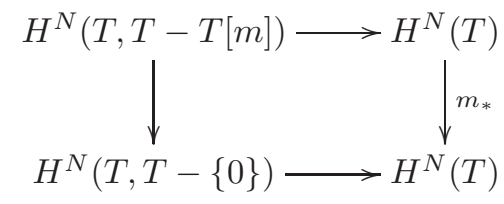

is commutative. The corresponding map

$$
H^{0}(T[m]) \rightarrow \mathbf{Z}
$$

is induced by the summation map

$$
\Sigma: \mathbf{Z}[T[m]] \rightarrow \mathbf{Z ;} \quad\left(a_{t}\right)_{t \in T[m]} \mapsto \sum_{t \in T[m]} a_{t} .
$$

The class $\left[T[m]-m^{N}\{0\}\right] \in H^{0}(T[m])$ corresponds to an element in $\mathbf{Z}[T[m]]^{0}=\operatorname{ker}(\Sigma)$, and to any point $x \in\left(\mathbf{Z}[T[m]]^{0}\right)^{\Gamma}$ it corresponds a class in $H^{N}(T, T-T[m])$ whose image in $H^{N}(T)$ belongs to the kernel of $m_{*}$. Recall that, by (2.1), the latter is injective over the rationals. By Sullivan's observation, this class in $H^{N}(T, T-T[m], \mathbf{Q})$ may therefore be lifted to a class in $H^{N-1}(T-T[m], \mathbf{Q})$. Here again, at this stage, such a lift is only defined up to $H^{N-1}(T, \mathbf{Q})$. 
2. Beilinson, Kings and Levin [2] propose to not use $\mathbf{Q}$ coefficients but the so called logarithm sheaf $\log$ on $T$. The point of introducing the latter is to make the lift of the Thom class canonical. Indeed, the cohomology of $T$ with coefficients in $\mathcal{L} o g$ is concentrated in degree $N$ so that $H^{N-1}(T, \mathcal{L} \circ g)=0$. This gives nice integrality statements at the cost of losing some topological intuition.

3.2. A canonical lift. To pick a canonical lift $z_{m} \in H^{N-1}(T-T[m], \mathbf{Q})$ of the image of $\left[T[m]-m^{N}\{0\}\right] \in H^{0}(T[m])$ in $H^{N}(T, T-T[m])$ we will again consider a multiplication map in the fibers. Let $a$ be an integer coprime to $m$. Then multiplication by $a$ induces a map of pairs of spaces

$$
(T, T[a m]) \rightarrow(T, T[m])
$$

The inclusion map $i$ also induces a map of pairs of spaces

$$
(T, T[a m]) \rightarrow(T, T[m])
$$

and, by a slight abuse of notations, we denote by $a_{*}$ the self-maps in cohomology obtained by pre-composing the direct image maps $a_{*}$ with $i^{*}$, so that in what follows $a_{*}=a_{*} \circ i^{*}$. We then obtain the following commutative diagram:

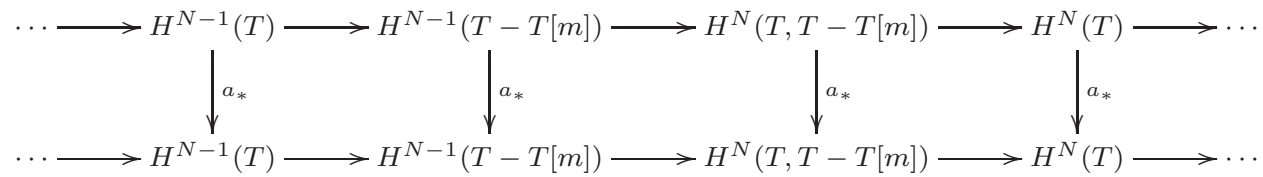

Now consider the Leray spectral sequence associated to the fibration $\pi: T \rightarrow M$ :

$$
E_{2}^{i, j}=H^{i}\left(M, R^{j} \pi_{*} \mathbf{Z}\right) \Rightarrow H^{i+j}(T)
$$

over the rationals. Since $a$ acts by multiplication on the fibers, it acts on $E_{2}^{i, j}$ by $a^{j}$. From that one deduces that all the differentials (on the second and any latter page) vanish, i.e. the spectral sequence degenerates on the second page. Moreover the $a^{j}$-eigenspace of the action of $a^{*}$ on $H^{i+j}(T, \mathbf{Q})$ is naturally identified with $H^{i}\left(M, R^{j} \pi_{*} \mathbf{Q}\right){ }^{4}$

It follows from (2.1) that $a_{*}$ acts by $a^{N-j}$ on the subspace $H^{i}\left(M, R^{j} \pi_{*} \mathbf{Q}\right) \subset H^{i+j}(A, \mathbf{Q})$. In particular the operator

$$
P\left(a_{*}\right)=\prod_{j=0}^{N-1}\left(a_{*}-a^{N-j}\right)
$$

acting on $H^{\bullet}(T, \mathbf{Q})$ annihilates

$$
\oplus_{j \leq N-1} H^{\bullet}\left(M, R^{j} \pi_{*} \mathbf{Q}\right)
$$

It thus acts trivially on $H^{N-1}(T, \mathbf{Q})$. We therefore obtain the following commutative diagram:

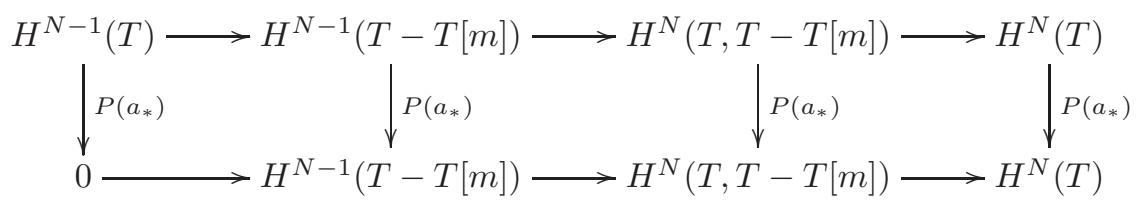

Now, since $a$ is coprime to $m$, we have $a_{*}\left(\left[T[m]-m^{N}\{0\}\right)=\left[T[m]-m^{N}\{0\}\right]\right.$ and

$$
P\left(a_{*}\right)\left(\left[T[m]-m^{N}\{0\}\right)=\left(\prod_{j=0}^{N-1}\left(1-a^{N-j}\right)\right)\left[T[m]-m^{N}\{0\}\right]\right.
$$

\footnotetext{
${ }^{4}$ This argument is sometimes referred to as 'Lieberman's trick.'
} 
is a nonzero multiple of $\left[T[m]-m^{N}\{0\}\right]$. If $z \in H^{N-1}(T-T[m], \mathbf{Q})$ is any lift of the image of $\left[T[m]-m^{N}\{0\}\right] \in H^{0}(T[m])$ in $H^{N}(T, T-T[m])$ then also is

$$
\frac{1}{\prod_{j=0}^{N-1}\left(1-a^{N-j}\right)} P\left(a_{*}\right)(z) \in H^{N-1}(T-T[m], \mathbf{Q}) .
$$

The latter is independent on the choice of $a$ since the operators $P\left(a_{*}\right)$ commute for different $a$ 's.

Definition 10. Let

$$
z_{m} \in H^{N-1}(T-T[m], \mathbf{Q})
$$

be the resulting canonical class.

Remark. As in the case of Sullivan's observation, for any integer $\ell$ coprime to $m$, we have:

$$
z_{m} \in H^{N-1}\left(T-T[m], \mathbf{Z}_{\ell}\right) \text {. }
$$

This follows from the proof by taking $a=\ell$ and noticing that $\prod_{j=0}^{N-1}\left(1-\ell^{N-j}\right)$ is coprime to $\ell$.

3.3. A canonical degree $N-1$ class on $M$. The class $z_{m}$ of Definition 10 depends on the choice of $m$. However if $m_{1}$ and $m_{2}$ are two integers, in $H^{0}\left(T\left[m_{1} m_{2}\right]\right)$ we have

$$
m_{1}^{*}\left[T\left[m_{2}\right]\right]=\left[T\left[m_{1} m_{2}\right]\right] \quad \text { and } m_{2}^{*}\left[T\left[m_{1}\right]\right]=\left[T\left[m_{1} m_{2}\right]\right]
$$

and Equation (2.1) implies:

$$
\begin{aligned}
{\left[T\left[m_{1} m_{2}\right]-\left(m_{1} m_{2}\right)^{N}\{0\}\right] } & =m_{1}^{*}\left[T\left[m_{2}\right]-m_{2}^{N}\{0\}+m_{2}^{N}\left[T\left[m_{1}\right]-m_{1}^{N}\{0\}\right]\right. \\
& =m_{2}^{*}\left[T\left[m_{1}\right]-m_{1}^{N}\{0\}+m_{1}^{N}\left[T\left[m_{2}\right]-m_{2}^{N}\{0\}\right] .\right.
\end{aligned}
$$

Since $z_{m_{1} m_{2}} \in H^{N-1}\left(T-T\left[m_{1} m_{2}\right], \mathbf{Q}\right)$ is determined by $\left[T\left[m_{1} m_{2}\right]-\left(m_{1} m_{2}\right)^{N}\{0\}\right]$ in $H^{0}\left(T\left[m_{1} m_{2}\right]\right)$ we conclude that

(3.2) $m_{1}^{*}\left(z_{m_{2}}\right)+m_{2}^{N} z_{m_{1}}=m_{2}^{*}\left(z_{m_{1}}\right)+m_{1}^{N} z_{m_{2}}=z_{m_{1} m_{2}} \in H^{N-1}\left(T-T\left[m_{1} m_{2}\right], \mathbf{Q}\right)$.

Now if $x: M \rightarrow T$ is a torsion section such that $m_{1} x=x$ and $m_{2} x=x$ the inverse maps on cohomology $x^{*}, m_{1}^{*}$ and $m_{2}^{*}$ satisfy:

$$
x^{*}=x^{*} m_{1}^{*}=x^{*} m_{2}^{*},
$$

and it follows from Equation (3.2) that

$$
\left(1-m_{1}^{N}\right) x^{*}\left(z_{m_{1}}\right)=\left(1-m_{2}^{N}\right) x^{*}\left(z_{m_{2}}\right) .
$$

This motivates the following:

Definition 11. Let $x: M \rightarrow T$ be a torsion section of order coprime to $m$. We set

$$
z_{m}(x)=x^{*}\left(z_{m}\right) \in H^{N-1}(M, \mathbf{Q}) .
$$

For any $m$ such that $m x=x$, let

$$
z(x)=\frac{1}{m^{N}-1} z_{m}(x) \in H^{N-1}(M, \mathbf{Q}) .
$$

Remark. Here again, for any integer $\ell$ coprime to $m$, we have:

$$
z_{m}(x) \in H^{N-1}\left(M, \mathbf{Z}_{\ell}\right) .
$$




\section{THE UNIVERSAL SPACE OF ORIENTED QUADRATIC SPACES}

We shall consider the Eisenstein class on a particular torus bundle - the universal torus over a congruence quotient of the symmetric space associated to $\mathrm{SL}_{N}(\mathbf{R})$. In this section, we first define the universal space of oriented quadratic spaces and give some of its basic properties that will be relevant to us.

4.1. The space of oriented quadratic spaces. Let $N \geq 2$ be a positive integer and let $V=\mathbf{Q}^{N}$ (column vectors). Fix an orientation $o$ of $V(\mathbf{R})$ and denote by $Q$ the standard positive quadratic form on $V(\mathbf{R})$ defined by

$$
Q(x)={ }^{t} x \cdot x=x_{1}^{2}+\cdots+x_{N}^{2} .
$$

We will often abuse notations and denote by $V$ the real vector space $V(\mathbf{R})$. Denote respectively by $\mathfrak{g}=\operatorname{End}(V)$ and $\mathfrak{k}=\mathfrak{s o}(V)=\wedge^{2} V$ the Lie algebras of $\mathrm{GL}(V)=\mathrm{GL}_{N}(\mathbf{R})$ and $\mathrm{SO}(V)=\mathrm{SO}_{N}$, and let $\mathfrak{m}=S^{2} V \subset \operatorname{End}(V)$ be the subspace of symmetric matrices. We have

$$
\mathfrak{g}=\mathfrak{k} \oplus \mathfrak{m}
$$

and the decomposition is orthogonal with respect to the Killing form of $\mathfrak{g}$.

Given a matrix $A \in \mathrm{GL}_{N}(\mathbf{R})$, define a positive definite quadratic form $Q_{A}$ on $V$ by $Q_{A}(x)=Q\left(A^{-1} x\right)$; in the standard basis of $V$ this quadratic form is given by the symmetric matrix $\left(A^{\top}\right)^{-1} A^{-1}$. We define also an orientation $o_{A}=\operatorname{sgn}(\operatorname{det} A) \cdot o$ of $V$. The assignment $A \mapsto\left(Q_{A}, o_{A}\right)$ defines a bijection

$$
S:=\mathrm{GL}_{N}(\mathbf{R}) / \mathrm{SO}_{N} \simeq\left\{\left(Q^{\prime}, o^{\prime}\right) \mid \begin{array}{c}
Q^{\prime} \text { a positive definite quadratic form on } V \\
o^{\prime} \text { an orientation of } V(\mathbf{R})
\end{array}\right\} .
$$

4.2. The universal space of oriented quadratic spaces. The standard representation of $\mathrm{GL}_{N}(\mathbf{R})$ on $V$ makes the real vector bundle

$$
E=S \times V \stackrel{\pi}{\rightarrow} \mathrm{GL}_{N}(\mathbf{R}) / \mathrm{SO}_{N}
$$

$\mathrm{GL}_{N}(\mathbf{R})$-equivariant (the isomorphism $E \stackrel{\sim}{\rightarrow} g^{*} E$ for $g \in \mathrm{GL}_{N}(\mathbf{R})$ is given on each fiber by multiplication by $g$ ).

The bundle $E$ carries:

- a natural orientation $o_{E}=\left(o_{E_{x}}\right)_{x}$, and

- a metric $h_{E}=\left(h_{E_{x}}\right)_{x}$.

Indeed: if $x \in S$ is represented by $A \in \mathrm{GL}_{N}(\mathbf{R})$, then $o_{E_{x}}:=o_{A}$ and $h_{E_{x}}:=Q_{A}$. Both $o_{E}$ and $h_{E}$ are $\mathrm{GL}_{N}(\mathbf{R})$-equivariant.

We shall refer to the total space of $E$ as the universal family of oriented quadratic $N$ spaces.

4.3. The Maurer-Cartan connection on the linear group. On the linear group $\mathrm{GL}_{N}(\mathbf{R})$ (as on any Lie group) we have a distinguished differential 1-form - the Maurer-Cartan form - that takes its values in the Lie algebra $\mathfrak{g}$ and carries the basic infinitesimal information about the structure of $\mathrm{GL}_{N}(\mathbf{R})$. The Lie algebra $\mathfrak{g}$ is identified with the tangent space of $\mathrm{GL}_{N}(\mathbf{R})$ at the identity, and the Maurer-Cartan 1-form, which is a linear mapping

$$
T_{g} \mathrm{GL}_{N}(\mathbf{R}) \rightarrow T_{e} \mathrm{GL}_{N}(\mathbf{R})=\mathfrak{g}
$$

is given by the push forward along the left-translation in the group:

$$
X \mapsto\left(L_{g^{-1}}\right)_{*} X, \quad\left(X \in T_{g} \mathrm{GL}_{N}(\mathbf{R})\right) .
$$


One can write this form explicitly as

$$
g^{-1} d g
$$

it is a $\mathrm{GL}_{N}(\mathbf{R})$-invariant differential 1-form defined globally on the linear group and which takes values in $\mathfrak{g}$.

4.4. The linear group as a $\mathrm{SO}_{N}$-principal bundle. The quotient map $\mathrm{GL}_{N}(\mathbf{R}) \rightarrow S$ induces the structure of an $\mathrm{SO}_{N}$-principal bundle over $S$. We denote this bundle by $P$.

The Maurer-Cartan form canonically identifies the space

$$
A^{1}(P)^{\mathrm{GL}_{N}(\mathbf{R})}
$$

of $\mathrm{GL}_{N}(\mathbf{R})$-invariant differential 1-forms on $P$ with $\mathfrak{g}^{*}$ through the map:

$$
\mathfrak{g}^{*} \rightarrow A^{1}(P)^{\mathrm{GL}_{N}(\mathbf{R})} ; \quad L \mapsto L\left(g^{-1} d g\right) .
$$

It more generally identifies $A^{\bullet}(P)^{\mathrm{GL}_{N}(\mathbf{R})}$ with $\wedge^{\bullet} \mathfrak{g}^{*}$.

A $\mathrm{SO}_{N}$-connection on $P$ is a $\mathfrak{k}$-valued 1 -form $\theta \in A^{1}(P) \otimes \mathfrak{k}$ satisfying

$$
\begin{aligned}
\operatorname{Ad}(k)\left(k^{*} \theta\right) & =\theta, \quad k \in K, \\
\iota_{X} \theta & =X, \quad X \in \mathfrak{k} .
\end{aligned}
$$

Here $\mathfrak{k}$ denotes the Lie algebra of $\mathrm{SO}_{N}$. Note that $\mathrm{GL}_{N}(\mathbf{R})$ acts on $P$ and $S$ by left multiplication and the map $P \rightarrow S$ is $\mathrm{GL}_{N}(\mathbf{R})$-equivariant. We fix a $\mathrm{GL}_{N}(\mathbf{R})$-invariant $\mathrm{SO}_{N}$-connection $\theta$ on $P$ as follows: under the isomorphisms

$$
\left(A^{1}(P) \otimes \mathfrak{k}\right)^{\mathrm{GL}_{N}(\mathbf{R}) \times \mathrm{SO}_{N}} \simeq\left(\mathfrak{g}^{*} \otimes \mathfrak{k}\right)^{\mathrm{SO}_{N}} \simeq \mathrm{Hom}_{\mathrm{SO}_{N}}(\mathfrak{g}, \mathfrak{k}),
$$

the $\mathrm{GL}_{N}(\mathbf{R})$-invariant $\mathrm{SO}_{N}$-connections correspond to the $\mathrm{SO}_{N}$-equivariant sections of the inclusion map $\mathfrak{k} \hookrightarrow \mathfrak{g}$. We define $\theta$ to be the connection corresponding to the projection $p: \mathfrak{g} \rightarrow \mathfrak{k}$ with kernel $\mathfrak{p}$, or more explicitly

Its curvature

$$
\theta=p\left(g^{-1} d g\right)=\frac{1}{2}\left(g^{-1} d g-d\left({ }^{t} \bar{g}\right)^{t} \bar{g}^{-1}\right) .
$$

$$
\Omega=\left(\Omega_{i j}\right)_{1 \leq i, j \leq N}=d \theta+\theta^{2} \in A^{2}(P) \otimes \mathfrak{k}
$$

is a $\mathrm{GL}_{N}(\mathbf{R})$-invariant, $\mathfrak{k}$-valued 2 -form on $P$.

4.5. A natural metric connection on $E$. For any linear representation $W$ of $\mathrm{SO}_{N}$ there is an associated vector bundle $P \times{ }^{\mathrm{SO}_{N}} W$ over $S$, and a principal $\mathrm{SO}_{N}$-connection on $P$ induces a connection on any such vector bundle. It can be defined using the fact that the space of sections of $P \times \mathrm{SO}_{N} W$ over $S$ is isomorphic to the space of $\mathrm{SO}_{N}$-equivariant $W$-valued functions on $P$. More generally, the space of $k$-forms with values in $P \times{ }^{\mathrm{SO}_{N}} W$ is identified with the space of $\mathrm{GL}_{N}(\mathbf{R})$-equivariant and horizontal $W$-valued $k$-forms on $P$.

This applies in particular to the standard representation of $\mathrm{SO}_{N}$ on $V$. The corresponding bundle is

$$
P \times \mathrm{SO}_{N} V=\left(\mathrm{GL}_{N}(\mathbf{R}) \times V\right) / \mathrm{SO}_{N},
$$

where the (right) $\mathrm{SO}_{N}$-action on $\mathrm{GL}_{N}(\mathbf{R}) \times V$ is given by

$$
(g, v) \stackrel{k}{\mapsto}\left(g k^{-1}, k v\right) .
$$

We shall equip the bundle $P \times{ }^{\mathrm{SO}_{N}} V$ with the connection induced from that of $P$.

The linear group acts on $P \times{ }^{\mathrm{SO}_{N}} V$ by

$$
[g, v] \stackrel{h}{\mapsto}[h g, v]
$$


and turns $P \times{ }^{\mathrm{SO}_{N}} V$ into a $\mathrm{GL}_{N}(\mathbf{R})$-equivariant bundle over $S$.

The bundle $E$ is $\mathrm{GL}_{N}(\mathbf{R})$-equivariantly isomorphic to $P \times{ }^{\mathrm{SO}_{N}} V$ via the map:

$$
\Phi: E \rightarrow P \times{ }^{\mathrm{SO}_{N}} V ;([g], v) \mapsto\left[g, g^{-1} v\right] .
$$

We endow $E$ with the induced connection $\nabla$; it preserves the metric and the orientation of E.

\section{MATHAI-QUiLlen THOM FORM ON THE UNIVERSAL SPACE OF ORIENTED QUADRATIC SPACES}

5.1. The Mathai-Quillen universal Thom form. Mathai and Quillen [39, Theorem 6.4] have constructed an equivariant form in $A_{\mathrm{SO}_{N}}^{N}(V)$ that is closed and is universal in the sense that for any oriented real rank $N$ vector bundle $\mathcal{E}$ equipped with compatible metric and connection, the Chern-Weil homomorphism

$$
A_{\mathrm{SO}_{N}}^{\bullet}(V) \rightarrow A^{\bullet}(\mathcal{E})
$$

maps the Mathai-Quillen form to a differential form representing the Thom class of $\mathcal{E}$.

We shall apply this to the bundle $P \times{ }^{\mathrm{SO}_{N}} V$. First note that

$$
A^{\bullet}\left(P \times{ }^{\mathrm{SO}_{N}} V\right)=A^{\bullet}(P \times V)^{\mathrm{SO}_{N}} .
$$

Using the invariant connection on $P$ we identify the space $A^{\bullet}(P \times V)$ with

$$
C^{\infty}\left(\mathrm{GL}_{N}(\mathbf{R}) \times V, \wedge^{\bullet}(\mathfrak{g} \oplus V)^{*}\right) .
$$

The group $\mathrm{SO}_{N}$ acts on $\mathfrak{g}$ by the adjoint representation and acts linearly on $V$. These actions yield a natural action of $\mathrm{SO}_{N}$ on $\wedge \bullet(\mathfrak{g} \oplus V)^{*}$ that we denote by $\rho$. The $\mathrm{SO}_{N}$-action on (5.1) is then given by

$$
f \stackrel{k}{\mapsto}\left((g, v) \mapsto \rho(k)\left(f\left(g k, k^{-1} v\right)\right)\right) .
$$

The space $A^{\bullet}\left(P \times{ }^{\mathrm{SO}_{N}} V\right)$ is therefore identified with

$$
\left\{\begin{array}{l|l}
f: \mathrm{GL}_{N}(\mathbf{R}) \times V \stackrel{C^{\infty}}{\rightarrow} \wedge \bullet(\mathfrak{g} \oplus V)^{*} & \begin{array}{l}
f\left(g k, k^{-1} v\right)=\rho\left(k^{-1}\right)(f(g, v)), \\
\left(g \in \mathrm{GL}_{N}(\mathbf{R}), v \in V, k \in \mathrm{SO}_{N}\right)
\end{array}
\end{array}\right\} .
$$

The $\mathrm{GL}_{N}(\mathbf{R})$-action on the bundle $P \times{ }^{\mathrm{SO}_{N}} V$ yields an action on $A^{\bullet}\left(P \times{ }^{\mathrm{SO}_{N}} V\right)$, and the space $A^{\bullet}\left(P \times \mathrm{SO}_{N} V\right)^{\mathrm{GL}_{N}(\mathbf{R})}$ of invariant forms is identified with

$$
\left\{\begin{array}{l|l}
f: V \stackrel{C^{\infty}}{\rightarrow} \wedge \bullet(\mathfrak{g} \oplus V)^{*} \otimes^{N} V & \begin{array}{l}
f\left(k^{-1} v\right)=\rho\left(k^{-1}\right)(f(v)) \\
\left(v \in V, k \in \mathrm{SO}_{N}\right)
\end{array}
\end{array}\right\}
$$

The Chern-Weil homomorphism

$$
A_{\mathrm{SO}_{N}}^{\bullet}(V) \rightarrow A^{\bullet}\left(P \times{ }^{\mathrm{SO}_{N}} V\right)
$$

maps the Mathai-Quillen form to an element

$$
U \in\left[\mathcal{S}(V) \otimes \wedge^{N}(\mathfrak{g} \oplus V)^{*}\right]^{\mathrm{SO}_{N}} \subset A^{N}\left(P \times^{\mathrm{SO}_{N}} V\right)^{\mathrm{GL}_{N}(\mathbf{R})}
$$

that is rapidly decreasing as a function of $V$.

Mathai and Quillen compute explicitly their equivariant Thom form - see [39, Eq. (6.1)] (see also [26, 3]). In the rest of this section we shall essentially follow their lines to give an explicit formula for $U$. 
5.2. Some notation. First fix some notations: denote by $\langle\cdot, \cdot\rangle$ the canonical scalar product on $V$, and let $|\cdot|$ be the associated norm. Let $\left(e_{1}, \ldots, e_{N}\right)$ be an oriented orthonormal basis of $V$. We adopt the following convenient convention: given $I \subset\{1, \ldots, N\}$ of cardinal $|I|=k$, we denote by $e_{I}$ the monomials

$$
e_{i_{1}} \wedge \ldots \wedge e_{i_{k}}, I=\left\{i_{1}, \ldots, i_{k}\right\}, i_{1}<\ldots<i_{k}
$$

in the exterior algebra $\wedge^{\bullet} \mathrm{V}$. We denote by $I^{\prime}$ the complement of the subset $I$ and define the sign $\varepsilon\left(I, I^{\prime}\right)= \pm 1$ by

$$
e_{I} \wedge e_{I^{\prime}}=\varepsilon\left(I, I^{\prime}\right) e_{1} \wedge \ldots \wedge e_{N}
$$

We finally denote by $e^{I}$ the dual basis of $\wedge^{\bullet} V^{*}$ and let

$$
d x_{I} \in \operatorname{Hom}\left(\wedge^{|I|} V, \mathbf{R}\right)
$$

be the corresponding form.

Consider the space

$$
A^{i, j}:=\left[C^{\infty}(V) \otimes \wedge^{i}(\mathfrak{g} \oplus V)^{*} \otimes \wedge^{j} V\right]^{\mathrm{SO}_{\mathrm{N}}}
$$

of $\mathrm{GL}_{N}(\mathbf{R})$-invariant forms on $P \times \mathrm{SO}_{N} V$, with values in the bundle $\wedge^{\bullet} V$.

5.3. Some natural forms in $A^{\bullet \bullet \bullet}$. First write $\mathbf{v}$ for the identity map

$$
\mathbf{v} \in A^{0,1}=C^{\infty}(V, V)^{\mathrm{SO}_{N}} .
$$

Multiplying $\mathbf{v}$ by the connection form $\theta \in\left(\mathfrak{g}^{*} \otimes \mathfrak{k}\right)^{\mathrm{SO}_{N}}$ gives a element in $C^{\infty}\left(V, \mathfrak{g}^{*}\right) \otimes$ $V)^{\mathrm{SO}_{N}}$. Write

$$
d \mathbf{v}=\sum_{i=1}^{N} d x_{i} \otimes e_{i} \in C^{\infty}\left(V, V^{*} \otimes V\right)^{\mathrm{SO}_{\mathrm{N}}} .
$$

The covariant derivative of $\mathbf{v}$ with respect to our canonical invariant connection gives the following element in $A^{1,1}$ :

$$
\nabla \mathbf{v}=d \mathbf{v}+\theta \cdot \mathbf{v} \in A^{1,1}=\left[C^{\infty}\left(V, \mathfrak{g}^{*} \oplus V^{*}\right) \otimes V\right]^{\mathrm{SO}_{\mathrm{N}}} .
$$

Finally, the curvature form $\Omega$ defines an element in $\operatorname{Hom}_{\mathrm{SO}_{N}}\left(\wedge^{2} \mathfrak{g}, \mathfrak{k}\right)$. Identifying $\mathfrak{k}$ with $\wedge^{2} V$ we shall see $\Omega$ as a constant map

$$
\Omega \in A^{2,2}=C^{\infty}\left(V, \wedge^{2}(\mathfrak{g})^{*} \otimes \wedge^{2} V\right)^{\mathrm{SO}_{\mathrm{N}}} .
$$

Now define an operator

$$
\iota(\mathbf{v}): A^{i, j} \rightarrow A^{i, j-1}
$$

by the following properties:

(1) $\iota(\mathbf{v})=\langle\mathbf{v}, \cdot\rangle$ on $A^{0,1}$,

(2) $\iota(\mathbf{v})$ is a derivation, that is,

$$
\iota(\mathbf{v})(\alpha \wedge \beta)=(\iota(\mathbf{v}) \alpha) \wedge \beta+(-1)^{i+j} \alpha \wedge(\iota(\mathbf{v}) \beta)
$$

for $\alpha \in A^{i, j}$ and $\beta \in A^{k, l}$.

Consider the differential form

$$
\omega=\frac{1}{2}|\mathbf{v}|^{2}+\nabla \mathbf{v}+\Omega \in A^{0,0} \oplus A^{1,1} \oplus A^{2,2} .
$$

Since

the following formula holds:

$$
\nabla|\mathbf{v}|^{2}=-2 \iota(\mathbf{v}) \nabla \mathbf{v}
$$

$$
(\nabla+\iota(\mathbf{v})) \omega=0 .
$$


We then can form:

$$
\exp (\omega):=e^{-\frac{|\mathbf{v}|^{2}}{2}} \sum_{k=0}^{N} \frac{(-1)^{k}}{k !}(\nabla \mathbf{v}+\Omega)^{k} \in \bigoplus_{k=0}^{N} A^{k, k},
$$

where we adopt the usual sign convention

$$
\left(\alpha \otimes e_{I}\right) \wedge\left(\beta \otimes e_{I^{\prime}}\right)=(-1)^{|I| \operatorname{deg}(\beta)}(\alpha \wedge \beta) \otimes\left(e_{I} \wedge e_{I^{\prime}}\right) .
$$

5.4. Explicit computation of $U$. Since $V=\mathbf{R}^{N}$ comes equipped with its canonical structure of an oriented Euclidean vector space, there is a canonical map

$$
B: \wedge^{\bullet} V \rightarrow \mathbf{R},
$$

called Berezin integral, defined by projecting $\alpha \in \wedge^{\bullet} V$ onto the component of the monomial $e_{1} \wedge \ldots \wedge e_{N}$.

The Berezin integral extends to a linear form $B: A^{i, j} \rightarrow A^{i, 0}$ which vanishes unless $j=N$. There are obtained by composing the fonctions in $C^{\infty}\left(V, \wedge^{j} V\right)$ with $B$. Since $\nabla$ is compatible with the metric, we have:

$$
d \circ B=B \circ \nabla .
$$

Theorem 12 (Mathai-Quillen). The invariant differential form

$$
U=(-1)^{\frac{1}{2} N(N-1)}(2 \pi)^{-N / 2} e^{-\frac{|\mathbf{v}|^{2}}{2}} B\left(\sum_{k=0}^{N} \frac{(-1)^{k}}{k !}(\nabla \mathbf{v}+\Omega)^{k}\right) \in A^{N, 0}
$$

is a closed $N$-form on the total space of the bundle $P \times{ }^{\mathrm{SO}_{N}} V$, and has constant integral 1 along the fibers. In other words, $U$ is a Thom form for the bundle $P \times{ }^{\mathrm{SO}_{N}} V$.

Proof. The proof that $B(\exp (\omega))$ - and therefore $U$ - is closed is a consequence of (5.10) and (5.8):

$$
\begin{aligned}
d B(\exp (\omega)) & =B(\nabla \exp (\omega)) \\
& =B((\nabla+\iota(\mathbf{v})) \exp (\omega)) \\
& =B(\exp (\omega)(\nabla+\iota(\mathbf{v})) \omega)=0 .
\end{aligned}
$$

Since $U$ is an invariant form, it remains to compute the integral along the fiber $V$ over the base point. This is done with a little linear algebra: identifying the constant functions in $C^{\infty}(V, V)$ with $V$ we can think of $\nabla \mathbf{v}$ as an element in $V^{*} \otimes V$. Then $(\nabla \mathbf{v})^{k} \in$ $\wedge^{k}\left(V^{*} \otimes V\right)$ has trivial image under $B$ unless $k=N$. Then, applying the sign convention (5.9), we have:

$$
\begin{aligned}
(\nabla \mathbf{v})^{N} & =N !\left(d x_{1} \otimes e_{1}\right) \cdots\left(d x_{N} \otimes e_{N}\right) \\
& =(-1)^{N(N+1) / 2} N !\left(e_{1} \wedge \ldots \wedge e_{N}\right)\left(d x_{1} \wedge \ldots \wedge d x_{N}\right) .
\end{aligned}
$$

We conclude that along the fiber $V$ over the base point we have:

$$
\begin{aligned}
U & =\frac{(-1)^{\frac{1}{2} N(N-1)}}{(2 \pi)^{N / 2}} e^{-\frac{|\mathbf{v}|^{2}}{2}} B\left(\sum_{k=0}^{N} \frac{(-1)^{k}}{k !}\left(\sum_{i=1}^{N} d x_{i} \otimes e_{i}\right)^{k}\right) \\
& =\frac{(-1)^{\frac{1}{2} N(N-1)}}{(2 \pi)^{N / 2}} e^{-\frac{|\mathbf{v}|^{2}}{2}} \frac{(-1)^{N}}{N !} B\left((-1)^{N(N+1) / 2} N !\left(e_{1} \wedge \ldots \wedge e_{N}\right)\right) d x_{1} \wedge \ldots \wedge d x_{N} \\
& =(2 \pi)^{-N / 2} e^{-\frac{|\mathbf{v}|^{2}}{2}} d x_{1} \wedge \ldots \wedge d x_{N} .
\end{aligned}
$$


Since the Gaussian integral $\int_{-\infty}^{+\infty} e^{-x^{2} / 2} d x=\sqrt{2 \pi}$ we conclude that the total integral of $U$ along the fiber is indeed equal to 1 .

5.5. Pfaffian forms. We may express the invariant form $U$ in terms of Pfaffian forms.

First assume that $N=2 \ell$ is even. A skew-symmetric matrix $A \in \mathfrak{k}=\mathfrak{s o}(V)$ can be identified with an element $A \in \wedge^{2} V$. Then (following Quillen)

$$
\frac{A^{\wedge \ell}}{\ell !}=\operatorname{Pf}(A) \cdot e_{1} \wedge \ldots \wedge e_{N}
$$

gives the Pfaffian. We similarly denote by $\operatorname{Pf}(\Omega)$ the $N$-form in $\operatorname{Hom}_{\mathrm{SO}_{N}}\left(\wedge^{N} \mathfrak{g}, \mathbf{R}\right)$ :

$$
A_{1} \wedge \cdots \wedge A_{N} \mapsto \sum_{\sigma \in \mathfrak{S}_{N}} \operatorname{sign}(\sigma) \operatorname{Pf}\left(\left[A_{\sigma(1)}, A_{\sigma(2)}\right], \ldots,\left[A_{\sigma(N-1)}, A_{\sigma(N)}\right]\right)
$$

Considered as a constant function of $V$, it defines an element

$$
\operatorname{Pf}(\Omega) \in A^{N, 0}=C^{\infty}\left(V, \wedge^{2}(\mathfrak{g} \oplus V)^{*}\right)^{\mathrm{SO}_{\mathrm{N}}} .
$$

For general $N$ we similarly construct forms in $A^{2 k, N-2 k}(0 \leq k \leq N)$. To any subset $I \subset\{1, \ldots, N\}$ with $|I|=2 k$ even, it indeed corresponds a decomposition $V=V_{I} \oplus V_{I^{\prime}}$, where say $V_{I}$ is generated by the $e_{i}$ 's $(i \in I)$. By restriction and projection to the subspace $V_{I}$ the curvature form $\Omega \in \operatorname{Hom}_{\mathrm{SO}(N)}\left(\wedge^{2} \mathfrak{g}, \mathfrak{s o}(V)\right)$ defines a form

$$
\Omega_{I} \in \operatorname{Hom}_{\mathrm{SO}(N)}\left(\wedge^{2} \mathfrak{g}, \mathfrak{s o}\left(V_{I}\right)\right)=\operatorname{Hom}_{\mathrm{SO}(N)}\left(\wedge^{2} \mathfrak{g}, \wedge^{2} V_{I}\right)
$$

Taking its $k$-th exterior product as above we define an alternating $|I|$-form

$$
\operatorname{Pf}\left(\Omega_{I}\right) \in \operatorname{Hom}_{\mathrm{SO}(N)}\left(\wedge^{|I|} \mathfrak{g}, \mathbf{R}\right) .
$$

And, being $\mathrm{SO}_{N}$-invariant, the sum

$$
\sum_{\substack{I \subset\{1, \ldots, N\} \\|I|=2 k}} \varepsilon\left(I, I^{\prime}\right) \operatorname{Pf}\left(\Omega_{I}\right) \otimes e_{I^{\prime}}
$$

considered as a constant function of $V$, defines a form in

$$
C^{\infty}\left(V, \wedge^{2 k}(\mathfrak{g} \oplus V)^{*} \otimes\left(\wedge^{N-2 k} V\right)^{*}\right)^{\mathrm{SO}_{\mathrm{N}}}=A^{2 k, N-2 k} .
$$

Theorem 13. The invariant Thom form

$$
U \in\left[\mathcal{S}(V) \otimes \wedge^{N}(\mathfrak{g} \oplus V)^{*}\right]^{\mathrm{SO}_{N}} \subset A^{N, 0}=A^{N}\left(P \times \mathrm{SO}_{N} V\right)^{\mathrm{GL}_{N}(\mathbf{R})}
$$

is given by

$$
U=(2 \pi)^{-N / 2} e^{-\frac{|\mathbf{v}|^{2}}{2}} \sum_{\substack{I \subset\{1, \ldots, N\} \\|I| \text { even }}} \varepsilon\left(I, I^{\prime}\right) \operatorname{Pf}\left(\Omega_{I}\right)(d \mathbf{v}+\theta \cdot \mathbf{v})^{I^{\prime}}
$$

Note that both sides of (5.14) are smooth functions on $V$ as is $|\mathbf{v}|^{2}$. Taking $v=0$, i.e. restricting to the zero section, gives the Euler form. 
5.6. The form on the universal bundle $E$. Recall that the $\mathrm{GL}_{N}(\mathbf{R})$-equivariant bundle (4.3) is $\mathrm{GL}_{N}(\mathbf{R})$-equivariantly isomorphic to $P \times{ }^{\mathrm{SO}_{N}} V$ via the map $\Phi$ (see (4.6)). By pull-back, this map induces a $\mathrm{GL}_{N}(\mathbf{R})$-equivariant isomorphism

$$
A^{\bullet}\left(P \times{ }^{\mathrm{SO}_{N}} V\right)^{\mathrm{GL}_{N}(\mathbf{R})} \stackrel{\sim}{\rightarrow} A^{\bullet}(E)^{\mathrm{GL}_{N}(\mathbf{R})} .
$$

We denote by

$$
\varphi \in\left[\mathcal{S}(V) \otimes \wedge^{N}(\mathfrak{m} \oplus V)^{*}\right]^{\mathrm{SO}_{N}} \subset A^{N}(E)^{\mathrm{GL}_{N}(\mathbf{R})}
$$

the pull-back $\Phi^{*} U$.

At a point $(e K, v)$ the differential of $\Phi$ maps an element $(X, w) \in \mathfrak{m} \oplus V$ to the class of the vector $(X, w-X v) \in \mathfrak{g} \oplus V$. It follows that the Schwartz function

$$
\varphi\left(\left(X_{1}+w_{1}\right) \wedge \ldots \wedge\left(X_{N}+w_{N}\right)\right) \in \mathcal{S}(V) \quad\left(X_{j}+w_{j} \in \mathfrak{m} \oplus V, j=1, \ldots, N\right)
$$

maps $v \in V$ to

$$
\left[U\left(\left(X_{1}+w_{1}-X_{1} v\right) \wedge \ldots \wedge\left(X_{N}+w_{N}-X_{N} v\right)\right)\right]\left(g^{-1} v\right) .
$$

5.7. The form as a $(\mathfrak{g}, K)$-cocycle. The linear group $\mathrm{GL}_{N}(\mathbf{R})$ acts on $\mathcal{S}(V)$ via the (Weil) representation $\omega$ :

$$
\omega(g): \mathcal{S}(V) \rightarrow \mathcal{S}(V) ; \phi \mapsto\left(v \mapsto \phi\left(g^{-1} v\right)\right) \quad\left(g \in \mathrm{GL}_{N}(\mathbf{R})\right) .
$$

This makes the bundle

$$
E \times \mathcal{S}(V) \rightarrow E
$$

$\mathrm{GL}_{N}(\mathbf{R})$-equivariant. We may therefore also think of

$$
\varphi \in\left[\mathcal{S}(V) \otimes \wedge^{N}(\mathfrak{m} \oplus V)^{*}\right]^{\mathrm{SO}_{N}} \cong\left[\mathcal{S}(V) \otimes A^{N}(E)\right]^{\mathrm{GL}_{N}(\mathbf{R})}
$$

as a $\mathrm{GL}_{N}(\mathbf{R})$-invariant $N$-form on the total space of $E$ with values in $\mathcal{S}(V)$. It follows that its restriction

$$
\bar{\varphi} \in \operatorname{Hom}_{\mathrm{SO}_{N}}\left(\wedge^{N} \mathfrak{m}, \mathcal{S}(V)\right)
$$

defines a $\left(\mathfrak{g l}_{N}, \mathrm{SO}_{N}\right)$-cocycle for the (Weil) representation $\omega$ of $\mathrm{GL}_{N}(\mathbf{R})$ on $\mathcal{S}(V)$.

For each $v_{0} \in V, \bar{\varphi}\left(v_{0}\right)$ is a closed invariant $N$-form on $S$; it is equal to the pull-back of the form $\varphi$ of (5.15) on $E$ by the flat section

$$
v_{0}: S \rightarrow S \times V ; x \mapsto\left(x, v_{0}\right) .
$$

\section{SOME EXPLicit FORMUlas FOR The MATHAI-QUillen ThOM FORM}

In applications we will need completely explicit formulas for the $N$-form (5.15). We consider three cases respectively associated to the groups $\mathrm{GL}_{1}(\mathbf{R}), \mathrm{GL}_{2}(\mathbf{R})$ and $\mathrm{GL}_{2}(\mathbf{C})$.

6.1. Explicit formula in the case $N=1$. This case amounts to consider the oriented line bundle $\pi: \mathbf{R} \rightarrow\{*\}$ with Euclidean metric $h(x)=x^{2}$. Then $\nabla \mathbf{v}=d x \otimes 1$ and we have

$$
\varphi=(2 \pi)^{-1 / 2} e^{-x^{2} / 2} \mathrm{~B}(\nabla \mathbf{v})=(2 \pi)^{-1 / 2} e^{-x^{2} / 2} d x \in A^{1}(\mathbf{R}) .
$$


6.2. Explicit formula in the case $N=2$. In this case the space $S$ is disconnected and we will rather work with the connected component

$$
S^{+}=\mathrm{GL}_{2}(\mathbf{R})^{+} / \mathrm{SO}_{2}
$$

the latter is identified with $\mathcal{H} \times \mathbf{R}_{>0}$ where

$$
\mathcal{H}=\mathcal{H}_{2}=\mathrm{GL}_{2}(\mathbf{R})^{+} / \mathrm{SO}_{2} Z(\mathbf{R})^{+}
$$

is the upper half-plane. We may coordinatize the $\mathrm{GL}_{2}(\mathbf{R})^{+}$-equivariant bundle $S^{+} \times \mathbf{R}^{2}$ as $\mathcal{H} \times \mathbf{R}_{>0} \times \mathbf{C}$.

Classical coordinates. Write

$$
(\tau=x+i y, t, z=u-v \tau) \in \mathcal{H} \times \mathbf{R}_{>0} \times \mathbf{C} .
$$

In these coordinates the left action of $\mathrm{GL}_{2}(\mathbf{R})^{+}$on $S^{+} \times \mathbf{R}^{2}$

$$
B \cdot(Q,(u, v))=\left(Q \circ B^{-1},(a u+b v, c u+d v)\right),
$$

where $B=\left(\begin{array}{ll}a & b \\ c & d\end{array}\right)$ and $B^{-1}=\left(\begin{array}{cc}d & -b \\ -c & a\end{array}\right)$, translates into:

$$
B:(\tau, t, z) \mapsto\left(\frac{a \tau+b}{c \tau+d}, \operatorname{det}(B)^{1 / 2} t, \frac{z}{c \tau+d}\right) .
$$

In particular, by restricting to the subspace $t=1$, we get a $\mathrm{SL}_{2}(\mathbf{R})$-equivariant embedding

$$
\mathcal{H} \times \mathbf{C} \rightarrow S^{+} \times \mathbf{R}^{2}
$$

where $(\tau, z)$ is mapped to

$$
\left(\frac{t}{\sqrt{y}}\left(\begin{array}{ll}
y & x \\
0 & 1
\end{array}\right),\left(\begin{array}{l}
u \\
v
\end{array}\right)\right) \in\left(\mathrm{GL}_{2}(\mathbf{R})^{+} / \mathrm{SO}_{2}\right) \times \mathbf{R}^{2} .
$$

The corresponding quadratic form $Q_{\tau}$ is associated to the symmetric matrix

$$
\frac{1}{y}\left(\begin{array}{cc}
1 & -x \\
-x & r^{2}
\end{array}\right)
$$

where we wrote, for short, $r^{2}=x^{2}+y^{2}$. It follows that the $Q_{\tau}$-norm of the vector $(u, v)$ is $y^{-1}|z|^{2}$, where $u-v \tau=z$. The metric on $\mathcal{H} \times \mathbf{C}$ is therefore given by

$$
\frac{|d \tau|^{2}}{y^{2}}+\frac{|d z|^{2}}{y}
$$

The invariant Thom form. We now compute the restriction of $\varphi$ to $\mathcal{H} \times \mathbf{C}$. To do so, we consider the $\mathrm{SL}_{2}(\mathbf{R})$-equivariant embedding

$$
\Phi:\left\{\begin{array}{ccc}
\mathcal{H} \times \mathbf{C} & \rightarrow & \mathrm{GL}_{2}(\mathbf{R})^{+} \times \mathrm{SO}_{2} \mathbf{C} \\
(\tau, z) & \mapsto & {\left[g_{\tau}, y^{-1 / 2} z\right]}
\end{array}\right.
$$

where, on the right side, we have identified $\mathbf{C}$ with $\mathbf{R}^{2}$ as fibers above $i \in \mathcal{H}$, i.e. $(u, v) \in$ $\mathbf{R}^{2} \mapsto u-i v \in \mathbf{C}, \tau=x+i y$ and $g_{\tau}=\left(\begin{array}{cc}\sqrt{y} & x / \sqrt{y} \\ 0 & 1 / \sqrt{y}\end{array}\right)$.

On $\mathrm{GL}_{2}(\mathbf{R})^{+} \times{ }^{\mathrm{SO}_{2}} \mathbf{C}$ the connection form $\theta$ is equal to $p\left(g^{-1} d g\right)$ (see $\left.\S 4.4\right)$ and, since

$$
g_{\tau}^{-1} d g_{\tau}=\left(\begin{array}{cc}
1 & 0 \\
0 & -1
\end{array}\right) \otimes \frac{d y}{2 y}+\left(\begin{array}{ll}
0 & 1 \\
0 & 0
\end{array}\right) \otimes \frac{d x}{y}
$$

the pull-back of $\theta$ by $\Phi$ is

$$
p\left(g_{\tau}^{-1} d g_{\tau}\right)=\left(\begin{array}{cc}
0 & 1 \\
-1 & 0
\end{array}\right) \otimes \frac{d x}{2 y} .
$$


We shall keep denoting by $\theta$ this connection form. In the coordinates $z=u-i v$, the matrix $\left(\begin{array}{cc}0 & 1 \\ -1 & 0\end{array}\right)$ acts on $\mathbf{R}^{2}$ like $i$ on $\mathbf{C}$. It follows that in complex coordinates we have

$$
\theta=\frac{i}{2} \frac{d x}{y}
$$

The (pullback of the) curvature form $\Omega=d \theta$ is then given by

$$
\Omega=-\frac{i}{2} \frac{d x \wedge d y}{y^{2}}
$$

Finally, in the coordinates $z=u-i v$, a skew-symmetric matrix $A \in \mathfrak{k}=\mathfrak{s o}_{2}$ acts on $\mathbf{R}^{2}=\mathbf{C}$ by multiplication by a purely imaginary complex number $z_{A}$, and

$$
\operatorname{Pf}(A)=i z_{A} .
$$

From all this we conclude that the Thom form $\varphi \in A^{2}(\mathcal{H} \times \mathbf{C})$ is given by

$$
\begin{aligned}
& \frac{1}{2 \pi} e^{-\frac{|z|^{2}}{2 y}}\left(\operatorname{Pf}(\Omega)-\frac{i}{2}\left(\left(d\left(y^{-1 / 2} z\right)+y^{-1 / 2} \theta z\right) \wedge\left(d\left(y^{-1 / 2} \bar{z}\right)-y^{-1 / 2} \theta \bar{z}\right)\right)\right) \\
= & \frac{1}{2 \pi} e^{-\frac{|z|^{2}}{2 y}}\left(\frac{1}{2} \frac{d x \wedge d y}{y^{2}}-\frac{i}{2}\left(\frac{d z}{y^{1 / 2}}+\frac{i}{2} \frac{z d \tau}{y^{3 / 2}}\right) \wedge\left(\frac{d \bar{z}}{y^{1 / 2}}-\frac{i}{2} \frac{\bar{z} d \bar{\tau}}{y^{3 / 2}}\right)\right) \\
= & \frac{1}{2 \pi} e^{-\frac{|z|^{2}}{2 y}}\left(\frac{1}{2} \frac{d x \wedge d y}{y^{2}}-\frac{1}{4} \frac{|z|^{2}}{y} \frac{d x \wedge d y}{y^{2}}+\frac{1}{4 y^{2}}(z d \tau \wedge d \bar{z}+\bar{z} d \bar{\tau} \wedge d z)-\frac{i d z \wedge d \bar{z}}{2 y}\right) .
\end{aligned}
$$

The Thom form is therefore given by:

$$
\varphi=\frac{1}{4 \pi} e^{-\frac{|z|^{2}}{2 y}}\left(\left(1-\frac{|z|^{2}}{2 y}\right) \frac{d x \wedge d y}{y^{2}}+\frac{1}{2 y^{2}}(z d \tau \wedge d \bar{z}+\bar{z} d \bar{\tau} \wedge d z)-\frac{i d z \wedge d \bar{z}}{y}\right) .
$$

6.3. Explicit computations in the $\mathrm{SL}_{2}(\mathbf{C})$-case. The symmetric space associated to the group $\mathrm{SL}_{2}(\mathbf{C})$ is the 3 -dimensional hyperbolic space. Let $\mathcal{H}_{3}$ be the upper half space model for the latter; it consists of all quaternion numbers

$$
r=z+j y \quad\left(z \in \mathbf{C}, y \in \mathbf{R}_{>0}, j^{2}=-1, i j=-j i\right) .
$$

We shall write $z(r)=z$ and $y(r)=y$.

Every element $\left(\begin{array}{ll}a & b \\ c & d\end{array}\right) \in \mathrm{SL}_{2}(\mathbf{C})$ acts on $\mathcal{H}_{3}$ by

$$
r \mapsto(a r+b)(c r+d)^{-1},
$$

where inversion and products are taken in the skew-field of quaternions.

Let us identify $\mathbf{C}^{2}$ with $\mathbf{R}^{4}$ via

$$
(u, v) \in \mathbf{C}^{2} \mapsto\left(\begin{array}{c}
\operatorname{Re}(u) \\
\operatorname{Re}(v) \\
\operatorname{Im}(u) \\
\operatorname{Im}(v)
\end{array}\right) \in \mathbf{R}^{4} .
$$

In this way we identify $\mathrm{SL}_{2}(\mathbf{C})$ as a subgroup of $\mathrm{GL}_{4}(\mathbf{R})$ via the embedding

$$
A+i B \mapsto\left(\begin{array}{cc}
A & -B \\
B & A
\end{array}\right) .
$$

This yields an embedding of the symmetric space $\mathcal{H}_{3}$ into the space $S=\mathrm{GL}_{4}(\mathbf{R}) / \mathrm{SO}_{4}$ of positive definite real quadratic forms on $\mathbf{C}^{2}$. The subspace $\mathcal{H}_{3}$ parametrizes positive 
definite Hermitian forms on $\mathbf{C}^{2}$ of discriminant 1 . The point $r=z+j y$ corresponds to the Hermitian form associated to the Hermitian matrix

$$
\frac{1}{y}\left(\begin{array}{cc}
1 & -z \\
-\bar{z} & y^{2}+|z|^{2}
\end{array}\right) .
$$

With respect to this Hermitian form, the norm of the vector $(u, v)$ is

$$
y^{-1}\left(|u|^{2}-(z \bar{u} v+\bar{z} u \bar{v})+\left(y^{2}+|z|^{2}\right)|v|^{2}\right)=y^{-1} \mathrm{~N}(u-r v),
$$

where $\mathrm{N}(\cdot)$ denotes the norm on quaternions. We coordinatize $\mathcal{H}_{3} \times \mathbf{C}^{2}$ as $(r=z+$ $j y,(u, v))$. In these coordinates the action of $\mathrm{SL}_{2}(\mathbf{C})$ on $\mathbf{C}^{2}$ is the usual (linear) one.

Consider the $\mathrm{SL}_{2}(\mathbf{C})$-equivariant embedding

$$
\Phi:\left\{\begin{array}{rlc}
\mathcal{H}_{3} \times \mathbf{C}^{2} & \rightarrow & \mathrm{GL}_{4}(\mathbf{R}) \times \mathrm{SO}_{4} \mathbf{C}^{2} \\
(r,(u, v)) & \mapsto & {\left[g_{r}, g_{r}^{-1}\left(\begin{array}{l}
u \\
v
\end{array}\right)\right]}
\end{array}\right.
$$

where $g_{r}=\left(\begin{array}{cc}y^{1 / 2} & y^{-1 / 2} z \\ 0 & y^{-1 / 2}\end{array}\right) \in \mathrm{SL}_{2}(\mathbf{C})$ - viewed as a matrix in $\mathrm{GL}_{4}(\mathbf{R})-$ maps $(0,1) \epsilon$ $\mathcal{H}_{3}$ to $r=(z, y)$, and $\mathbf{C}^{2}$ is identified with $\mathbf{R}^{4}$ as above.

Computations similar to those of the preceding paragraph lead to

$$
\theta=\frac{1}{2}\left(\begin{array}{cc}
0 & \frac{d z}{y} \\
-\frac{d \bar{z}}{y} & 0
\end{array}\right) \text { and } \Omega=\frac{1}{4}\left(\begin{array}{cc}
-\frac{d z \wedge d \bar{z}}{y} & -2 \frac{d y \wedge d z}{y^{2}} \\
2 \frac{d y \wedge d \bar{z}}{y^{2}} & \frac{d z \wedge \bar{z}}{y}
\end{array}\right),
$$

and we find that, in restriction to $\mathcal{H}_{3} \times \mathbf{C}^{2}$, the Thom form $\varphi$ is given by

$$
\begin{aligned}
\varphi=-\frac{1}{16 \pi^{2}} e^{-\frac{\mathrm{N}(u-r v)}{2 y}}\left[-\frac{d z \wedge d \bar{z}}{2 y}\right. & \wedge B \wedge \bar{B}-\frac{d y \wedge d \bar{z}}{y^{2}} \wedge A \wedge \bar{B} \\
+\frac{d y \wedge d z}{y^{2}} & \left.\wedge B \wedge \bar{A}+\frac{d z \wedge d \bar{z}}{2 y} \wedge A \wedge \bar{A}+C \wedge D \wedge \bar{D} \wedge \bar{C}\right]
\end{aligned}
$$

where

$$
\begin{gathered}
A=y^{-1 / 2}(d u-z d v)-\frac{1}{2} y^{-1 / 2} v d z, B=y^{1 / 2} d v-\frac{1}{2} y^{-3 / 2}(u-z v) d \bar{z}, \\
C=y^{-1 / 2}(d u-z d v)-\frac{1}{2} y^{-3 / 2}(u-z v) d y-\frac{1}{2} y^{-1 / 2} v d z
\end{gathered}
$$

and

$$
D=y^{1 / 2} d v+\frac{1}{2} y^{-1 / 2} v d y-\frac{1}{2} y^{-3 / 2}(u-z v) d \bar{z}
$$

\section{CANONICAL TRANSGRESSION OF THE THOM FORM}

Consider the Euler form $\bar{\varphi}(0)$ on $S$ as a $\mathrm{GL}_{N}(\mathbf{R})$-invariant form on $E$. Building on Chern's proof of the Gauss-Bonnet Theorem [18], Mathai and Quillen [39, §7] have constructed a primitive of $\bar{\varphi}(0)$ away from the zero section, i.e. on

$$
E-\{0\}:=S \times(V-\{0\}) .
$$

Once the metric on $V$ is fixed this transgression is canonical. In this section we briefly recall their construction and compute explicit formulas in each of the three cases considered in the previous section. We first recast the Mathai-Quillen construction in $\left(\mathfrak{g l}_{N}, \mathrm{SO}_{N}\right)$ cohomology of the Weil representation $\omega$. 
7.1. Relation with the Weil representation. The space $W=V \oplus V^{*}$ is naturally equipped with a symplectic form with respect to which $V$ and $V^{*}$ are isotropic and induces the canonical duality between $V$ and $V^{*}$. The symplectic group $\operatorname{Sp}(W)$ acts on $\mathcal{S}(V)$ through the Weil representation. Now $(\mathrm{GL}(V), \mathrm{GL}(1))$ forms a dual pair - in the sense of Howe [32] — in $\operatorname{Sp}(W)$, and the restriction of the Weil representation to $\mathrm{GL}(V)$ is precisely the representation $\omega$ of $\mathrm{GL}(V)$ in $\mathcal{S}(V)$ that we are considering.

Let $\mathrm{S}(V) \subset \mathcal{S}(V)$ be the polynomial Fock space. It consists of those Schwartz functions on $V$ that are of the form

$$
v \mapsto p(v) e^{-\frac{|\mathbf{v}|^{2}}{2}}
$$

where $p$ is a polynomial on $V$. These functions are the vectors in $\mathcal{S}(V)$ that span a finite dimensional subspace under the action of the maximal compact subgroup of the metaplectic group. The form $\varphi$ belongs to the polynomial Fock space.

7.2. A transgression of $\varphi$ in $\left(\mathfrak{g l}_{N}, \mathrm{SO}_{N}\right)$-cohomology. Let $L$ be the infinitesimal generator of the second factor GL(1) in the 'dual pair' $(\mathrm{GL}(V), \mathrm{GL}(1))$; it acts on $E=S \times V$ trivially on $S$ and linearly on $V$. In other words, it acts by multiplication by $t>0$ in the fibers. This gives a 1-parameter group of diffeomorphisms in the variable $s=\log t$.

Proposition 14. There exists

$$
\psi \in\left[\mathcal{S}(V) \otimes \wedge^{N-1}(\mathfrak{m} \oplus V)^{*}\right]^{\mathrm{SO}_{N}} \subset A^{N-1}(E)^{\mathrm{GL}_{N}(\mathbf{R})}
$$

such that

$$
\omega(L) \cdot \varphi=d \psi
$$

Proof. The invariant vector field

$$
X=0_{N} \oplus\left(v_{1} e_{1}+\ldots+v_{N} e_{N}\right)
$$

on $P \times{ }^{\mathrm{SO}_{N}} V$ is associated to $L$ and generates the flow associated to the one-parameter group of diffeomorphisms acting by multiplication on the fibers. Then we have:

$$
\begin{aligned}
\omega(L) \cdot \varphi & =\frac{d}{d s} t^{*} \varphi \\
& =\mathcal{L}_{X}\left(t^{*} \varphi\right) \\
& =d\left\{i_{X}\left(t^{*} \varphi\right)\right\} .
\end{aligned}
$$

We may therefore take $\psi=i_{X} \varphi$.

It follows from Theorem 12 that

$$
\psi=-(-1)^{\frac{1}{2} N(N-1)}(2 \pi)^{-N / 2} e^{-\frac{|\mathbf{v}|^{2}}{2}} B\left(\sum_{k=0}^{N} \frac{(-1)^{k}}{k !} \mathbf{v}(\nabla \mathbf{v}+\Omega)^{k}\right) \in A^{N-1,0} .
$$

Remark. We may rewrite equation (7.2) as:

$$
d\left(t^{*} \psi\right)=t \frac{d}{d t}\left(t^{*} \varphi\right) .
$$


7.3. Transgression of $\bar{\varphi}(0)$. As $t$ tends 0 the form $t^{*} \varphi$ tends to $\bar{\varphi}(0)$ (viewed as an invariant form on $E$ ), and, as $t$ tends to $+\infty, t^{*} \varphi$ tends to zero on $E-\{0\}$. Integrating the form

$$
-t^{*} \psi=-i_{X}\left(t^{*} \varphi\right)
$$

from zero to infinity gives a transgression of $\bar{\varphi}(0)$ away from the zero section. In fact one can prove:

Proposition 15. We have:

$$
d\left(\int_{0}^{+\infty} t^{*} \psi \frac{d t}{t}\right)=\delta_{0}-\bar{\varphi}(0) .
$$

Here $\delta_{0}$ is the current of integration along the 0 section of $E$.

\subsection{Explicit computations.}

The case $N=1$. Recall that this case amounts to consider the oriented line bundle $\pi$ : $\mathbf{R} \rightarrow\{*\}$ with Euclidean metric $h(x)=x^{2}$. It follows from (6.1) that $\bar{\varphi}(0)=0$ and that

$$
\psi_{(\mathbf{R}, h)}=(2 \pi)^{-1 / 2} e^{-x^{2} / 2} \mathrm{~B}(\mathbf{v})=-(2 \pi)^{-1 / 2} e^{-x^{2} / 2} x \in A^{0}(\mathbf{R}) .
$$

One easily checks that

$$
-\int_{0}^{+\infty} t^{*} \psi \frac{d t}{t}=\frac{1}{2}
$$

which is obviously a primitive of 0 .

The case $N=2$. Using the coordinates $(\tau, z)$ of $\S 6.2$, the vector field $X$ on $\mathcal{H} \times \mathbf{C}$ becomes

$$
X=z \frac{\partial}{\partial z}+\bar{z} \frac{\partial}{\partial \bar{z}}
$$

It then follows from (6.5) that the form $\psi=i_{X} \varphi$ is equal to

$$
-\frac{1}{4 \pi} e^{-\frac{|z|^{2}}{2 y}}\left(\frac{1}{2 y^{2}}\left(|z|^{2} d \tau+|z|^{2} d \bar{\tau}\right)+\frac{i}{y}(z d \bar{z}-\bar{z} d z)\right) .
$$

Rewriting $\psi$ as

$$
-\frac{1}{2 \pi}\left(\frac{|z|^{2}}{2 y} e^{-\frac{|z|^{2}}{2 y}}\right)\left(\frac{1}{2}\left(\frac{d \tau}{y}+\frac{d \bar{\tau}}{y}\right)+i\left(\frac{d \bar{z}}{\bar{z}}-\frac{d z}{z}\right)\right)
$$

and using that

$$
\int_{0}^{\infty} \frac{t^{2}|z|^{2}}{2 y} e^{-\frac{t^{2}|z|^{2}}{2 y}} \frac{d t}{t}=\frac{1}{2} \int_{0}^{\infty} u e^{-u} \frac{d u}{u}=\frac{1}{2},
$$

we get that the transgression form is

$$
-\int_{0}^{\infty} t^{*} \psi \frac{d t}{t}=\frac{1}{4 \pi}\left(\frac{1}{2}\left(\frac{d \tau}{y}+\frac{d \bar{\tau}}{y}\right)+i\left(\frac{d \bar{z}}{\bar{z}}-\frac{d z}{z}\right)\right) .
$$

One easily checks that the differential of the 1 -form (7.7) is

$$
\frac{1}{4 \pi y^{2}} d x \wedge d y=\bar{\varphi}(0) \text {. }
$$


The case $\mathrm{SL}_{2}(\mathbf{C})$. The total space is $\mathcal{H}_{3} \times \mathbf{C}^{2}$ and we use the coordinates $(r=z+$ $j y,(u, v))$ of $\S 6.3$. Rather the developing the full formula (6.7), we just identify the components of $\psi=\iota_{X} \varphi$ of types $d y \wedge d u \wedge d v, d z \wedge d u \wedge d v$ and $d \bar{z} \wedge d u \wedge d v$. We find:

$$
\begin{array}{r}
\psi=\frac{1}{2}\left(\frac{i}{2 \pi y}\right)^{2} e^{-\frac{\mathrm{N}(u-r v)}{2 y}}\left[(\bar{u}-\bar{z} \bar{v})^{2} d z-2(\bar{u}-\bar{z} \bar{v}) \bar{v} y d y-(\bar{v} y)^{2} d \bar{z}\right] \wedge d u \wedge d v \\
+(\text { other terms }) .
\end{array}
$$

\section{EISENSTEIN TRANSGRESSION}

In this section we come back to torus bundles. We consider the universal family of real tori - with level structure. We then globalize the Mathai-Quillen forms to construct an explicit transgression of the Chern-Weil differential form representing the Euler class. This refinement of Sullivan's Theorem 23 is due to Bismut and Cheeger [8]. We rephrase their construction in group theoretic terms using Howe's viewpoint on theta series.

8.1. Universal families of real tori. Let's identify the center $Z$ of $\mathrm{GL}_{N}$ with the multiplicative group $\mathbf{G}_{m}$ via the map $z \mapsto z \cdot 1_{N}$. Write $Z(\mathbf{R})^{+}$for the connected component of the identity in $Z(\mathbf{R})$. The quotient

$$
X=\mathrm{GL}_{N}(\mathbf{R})^{+} / \mathrm{SO}_{N} Z(\mathbf{R})^{+}=S^{+} / Z(\mathbf{R})^{+}
$$

is identified with a connected component of the quotient of $S$ by $Z(\mathbf{R})^{+}$. It is also the symmetric space associated to $\mathrm{SL}_{N}(\mathbf{R})$. The bundle $P \times{ }^{\mathrm{SO}_{N}} V$ induces a $\mathrm{GL}_{N}(\mathbf{R})^{+}$equivariant vector bundle of $X$. The latter is $\mathrm{SL}_{N}(\mathbf{R})$-equivariantly isomorphic to the $\mathrm{SL}_{N}(\mathbf{R})$-equivariant bundle

$$
X \times V \rightarrow X
$$

via the restriction of the map $\Phi$ (see (4.6)).

Now, let $L \subset V(\mathbf{Q})$ be a lattice, $m$ be a positive integer and $\Gamma$ be a subgroup of $\mathrm{GL}_{N}(\mathbf{Q})^{+}\left(:=\mathrm{GL}_{N}(\mathbf{Q}) \cap \mathrm{GL}_{N}(\mathbf{R})^{+}\right)$that fixes $L$. Consider the torus bundle

$$
X \times V / L \rightarrow X .
$$

We refer to its quotient

$$
T:=\Gamma \backslash(X \times V / L) \rightarrow \Gamma \backslash X
$$

as the universal family of tori with level structure $\Gamma$. The fundamental group of the total space $T$ is $\Gamma \ltimes L$.

8.2. The affine group action. The total space of the fiber bundle $E$ can be realized as a homogeneous space under the action of the affine group $\mathrm{GL}_{N}(\mathbf{R}) \ltimes V$ where

$$
(g, v) \cdot\left(g^{\prime}, v^{\prime}\right)=\left(g g^{\prime}, g v^{\prime}+v\right) .
$$

The left action of the affine group on $E$ is transitive and the stabilizer of the base point $(e K, 0)$ in $E$ is the group $\mathrm{SO}_{N}$ embedded in the affine group as

$$
\mathrm{SO}_{N} \hookrightarrow \mathrm{GL}_{N}(\mathbf{R}) \ltimes V ; k \mapsto(k, 0) .
$$

The Weil representation $\omega$ of $\mathrm{GL}_{N}(\mathbf{R})$ extends to a representation of the affine group on $\mathcal{S}(V)$ that we denote by ${ }^{5}$

$$
\omega(g, w): \mathcal{S}(V) \rightarrow \mathcal{S}(V) ; \phi \mapsto\left(v \mapsto \phi\left(g^{-1}(v-w)\right) \quad\left((g, w) \in \mathrm{GL}_{N}(\mathbf{R}) \ltimes V\right) .\right.
$$

\footnotetext{
${ }^{5}$ The group law in $\mathrm{GL}_{N}(\mathbf{R}) \ltimes V$ is $(g, w) \cdot\left(g^{\prime}, w^{\prime}\right)=\left(g g^{\prime}, g w^{\prime}+w\right)$ so that $(g, w)^{-1}=\left(g^{-1},-g^{-1} w\right)$. Finally $\mathrm{GL}_{N}(\mathbf{R}) \ltimes V$ acts on $V$ by $(g, w) \cdot v=g v+w$.
} 
This makes the bundle

$$
E \times \mathcal{S}(V) \rightarrow E=\left(\mathrm{GL}_{N}(\mathbf{R}) \ltimes V\right) / \mathrm{SO}_{N}
$$

$\left(\mathrm{GL}_{N}(\mathbf{R}) \ltimes V\right)$-equivariant. We may then think of $\varphi$ and $\psi$ as elements in

$$
\left[\mathcal{S}(V) \otimes \wedge^{\bullet}(\mathfrak{m} \oplus V)^{*}\right]^{\mathrm{SO}_{N}} \cong\left[\mathcal{S}(V) \otimes A^{\bullet}(E)\right]^{\mathrm{GL}_{N}(\mathbf{R}) \ltimes V},
$$

i.e. as $\left(\mathrm{GL}_{N}(\mathbf{R}) \ltimes V\right)$-invariant forms on the total space of $E$ with values in $\mathcal{S}(V)$. In particular $\varphi$ defines a $\left(\mathfrak{g l}_{N} \ltimes V, \mathrm{SO}_{N}\right)$-cocycle for $\omega$.

8.3. Theta series. On $\mathcal{S}(V)$ there is a distribution $\theta_{L}$ defined by

$$
\theta_{L}(\phi)=\sum_{\lambda \in L} \phi(\lambda)
$$

which satisfies

$$
\theta_{L}(\omega(\gamma, \lambda) \phi)=\theta_{L}(\phi) \quad((\gamma, \lambda) \in \Gamma \ltimes L) .
$$

For $\phi \in \mathcal{S}(V), g \in \mathrm{GL}_{N}(\mathbf{R})$ and $v \in V$, let

$$
\theta_{\phi}(g, v)=\theta_{L}(\omega(g, v) \cdot \phi)=\sum_{\lambda \in L} \phi\left(g^{-1}(\lambda-v)\right) .
$$

Clearly this is invariant under $v \mapsto v+\lambda$ for $\lambda \in L$. It is also invariant under $\Gamma \ltimes L$. In particular we get two forms:

$$
\theta_{\varphi} \in A^{N}(E)^{\Gamma \ltimes L}
$$

and

$$
\theta_{\psi} \in A^{N-1}(E)^{\Gamma \ltimes L} .
$$

Proposition 16. The differential form $\theta_{\varphi}$ represents a Thom form for the torus bundle $\Gamma \backslash\left(S^{+} \times V / L\right) \rightarrow \Gamma \backslash S^{+}$.

Proof. It suffices to check that $\theta_{\varphi}$ has integral 1 along the fibers. After unfolding the integral this follows from the fact that $\varphi$ is a Thom form.

Remarks. 1. For all positive $t$, the differential form $\theta_{t^{*} \varphi}$ also represents a Thom form for the torus bundle $\Gamma \backslash\left(S^{+} \times V / L\right) \rightarrow \Gamma \backslash S^{+}$, and as $t$ tends to infinity $\theta_{t^{*} \varphi}$ tends to 0 away from the zero section.

2. Let $v_{0} \in m^{-1} L$. Then translation by $v_{0}$ on $\Gamma \backslash\left(S^{+} \times V / L\right)$ maps the zero section of the torus bundle to the image $\left[v_{0}\right]$ of the torsion section

$$
\mathbf{v}_{0}: \Gamma \backslash S^{+} \rightarrow \Gamma \backslash\left(S^{+} \times V / L\right) ;[x] \mapsto\left[x, v_{0}\right]
$$

and the $N$-form

$$
\theta_{\varphi}\left(g, v-v_{0}\right)=\left[\omega\left(e, v_{0}\right)^{*} \theta_{\varphi}\right](g, v)
$$

represents the dual class $\left[v_{0}\right] \in H^{N}\left(\Gamma \backslash\left(S^{+} \times V / L\right)\right)$. 
8.4. Eisenstein regularization. The $(N-1)$-form $\theta_{t^{*} \psi}$ is obtained by averaging $t^{*} \psi$ under the action of $L$. Proposition 19 suggests to rather average the form

$$
\int_{0}^{+\infty} t^{*} \psi \frac{d t}{t}
$$

to get a transgression of the Euler class. Since (8.5) is not rapidly decreasing along the fibers, the averaging has to be done in a regularized way. Formally on wants to consider

$$
\int_{0}^{+\infty} \theta_{t^{*} \psi}(g, v) \frac{d t}{t}=\int_{0}^{+\infty} \theta_{\psi}\left(t^{-1} g, v\right) \frac{d t}{t}
$$

Here the identity follows from the $\mathrm{GL}_{N}(\mathbf{R})$-invariance of $\psi$, see (8.1). As long as $v \notin L$ this integral converges at $+\infty$. As usual we shall make sense of it in a regularized way by adding a power of $t$ under the integral. We recall the basic features of this regularization procedure in the following general proposition.

Consider a general function $\phi \in \mathcal{S}(V)$. Write

$$
\theta_{\phi}^{*}(g, v)= \begin{cases}\theta_{\phi}(g, v) & \text { if } v \notin L \\ \theta_{\phi}(g, v)-\phi(0) & \text { if } v \in L\end{cases}
$$

Proposition 17. On $\Gamma \backslash\left(S^{+} \times V / L\right)$ the expression

$$
E_{\phi}(g, v ; s)=\int_{0}^{+\infty} \theta_{t^{*} \phi}^{*}(g, v) t^{N s} \frac{d t}{t}=\int_{0}^{+\infty} \theta_{\phi}^{*}\left(t^{-1} g, v\right) t^{N s} \frac{d t}{t}
$$

is well defined for $\operatorname{Re}(s)>0$. It admits a meromorphic continuation to all $s \in \mathbf{C}$, and is holomorphic everywhere except at $s=0$ and $s=1$ where it has at most simple poles. It has a pole at zero only when $v \in L$.

Proof. The function

$$
t \mapsto \sum_{\lambda \in L}^{\prime} \phi\left(t g^{-1}(\lambda-v)\right)
$$

where the prime means that we sum over $\lambda \neq v$, is rapidly decreasing, and the integral

$$
\int_{1}^{+\infty}\left(\sum_{\lambda \in L}^{\prime} \phi\left(t g^{-1}(\lambda-v)\right)\right) t^{N s} \frac{d t}{t}
$$

is regular at every $s \in \mathbf{C}$. To deal with the integral from 0 to 1 we use Poisson summation formula. Since the Fourier transform of $\xi \mapsto f(g \xi)\left(g \in \mathrm{GL}_{N}(\mathbf{R})\right)$ is equal to $\xi \mapsto$ $|\operatorname{det} g|^{-1} f\left(\left(g^{\top}\right)^{-1} \xi\right)$, we have:

$$
\sum_{\lambda \in L} \phi\left(t g^{-1}(v+\lambda)\right)=\frac{|\operatorname{det} g|}{t^{N}} \sum_{\lambda \in L} \widehat{\phi}\left(t^{-1} g^{\top} \lambda\right) e^{2 i \pi v \cdot \lambda} .
$$


Using the change of variable $t \mapsto t^{-1}$, this implies that the integral

$$
\begin{aligned}
\int_{0}^{1}\left(\sum_{\lambda \in L}^{\prime} \phi\left(t g^{-1}(\lambda-v)\right)\right) t^{N s} \frac{d t}{t} \\
=|\operatorname{det} g| \int_{0}^{1}\left(\sum_{\lambda \in L} \widehat{\phi}\left(t^{-1} g^{\top} \lambda\right) e^{-2 i \pi v \cdot \lambda}-\delta_{L}(v) \frac{t^{N}}{|\operatorname{det} g|} \phi(0)\right) t^{N(s-1)} \frac{d t}{t} \\
=|\operatorname{det} g| \int_{1}^{\infty}\left(\sum_{\lambda \in L}^{\prime} \widehat{\phi}\left(t g^{\top} \lambda\right) e^{-2 i \pi v \cdot \lambda}\right) t^{N(1-s)} \frac{d t}{t} \\
+\frac{|\operatorname{det} g| \widehat{\phi}(0)}{N(s-1)}-\delta_{L}(v) \frac{\phi(0)}{N s} .
\end{aligned}
$$

is also regular at every $s \in \mathbf{C}-\{0,1\}$, has simple poles at $s=0$ and $s=1$, and only when $v \in L$ at $s=0$.

8.5. Eisenstein transgression. Since $\psi(0)=0$, it follows from Proposition 17 that the expression

$$
E_{\psi}(g, v):=E_{\psi}(g, v ; 0)
$$

is well defined. It is smooth away from the zero section and defines a $(N-1)$-form on $\Gamma \backslash\left(S^{+} \times(V / L-\{0\})\right)$.

The action of $Z(\mathbf{R})^{+}$on $S^{+}$extends trivially to an action on $S^{+} \times V$ which induces an action on $\Gamma \backslash\left(S^{+} \times V / L\right)$ whose quotient is the universal family $T$. We also have a natural action of $Z(\mathbf{R})^{+}$on differential forms on $S^{+} \times V$, and by the invariance properties (8.1) of $\psi$, for every $z \in Z(\mathbf{R})^{+}$we have

$$
\begin{aligned}
z^{*} E_{\psi}(s) & =\int_{0}^{+\infty} z^{*} \theta_{\psi}\left(t^{-1} g, v\right) t^{N s} \frac{d t}{t} \\
& =\int_{0}^{+\infty} \theta_{\psi}\left(t^{-1} z^{-1} g, v\right) t^{N s} \frac{d t}{t} \\
& =z^{-N s} E_{\psi}(s) .
\end{aligned}
$$

In particular $E_{\psi}$ is invariant by the center and defines a $(N-1)$-form on $T-\{0\}$.

In the theorem below we compute the differential of $E_{\psi}$ using (7.4). To state it we first need a definition.

Definition 18. The determinant

$$
\operatorname{det} \in\left[\wedge^{N} V^{*}\right]^{\mathrm{SO}_{N}} \cong A^{N}(E)^{\mathrm{GL}_{N}(\mathbf{R}) \ltimes V}
$$

defines a $\left(\mathrm{GL}_{N}(\mathbf{R}) \ltimes V\right)$-invariant $N$-form on the total space of $E$. It induces an $N$-form $\operatorname{vol}_{N}$ on the universal family $T$.

Restricted to a fiber the $N$-form $\operatorname{vol}_{N}$ is equal — up to constant factor — to the invariant probability volume form.

Theorem 19. The $(N-1)$-form $E_{\psi}$ is smooth and is a primitive of $-\operatorname{vol}_{N}$ on $T-\{0\}$. As a current on $T$ its differential $d E_{\psi}$ satisfies

$$
d E_{\psi}=\delta_{\{0\}}-\operatorname{vol}_{N} .
$$


Proof. By (7.2) if $v \notin L$ and $\operatorname{Re}(s)>1$, we have

$$
\begin{aligned}
d E_{\psi}(g, v ; s) & =\int_{0}^{+\infty} d \theta_{t^{*} \psi} t^{N s} \frac{d t}{t} \\
& =\int_{0}^{+\infty} \frac{d}{d t} \theta_{t^{*} \varphi} t^{N s} d t .
\end{aligned}
$$

For $\operatorname{Re}(s)>1$, partial integration applies to the last line of (8.11) and gives:

$$
d E_{\psi}(g, v ; s)=-N s \int_{0}^{+\infty} \theta_{t^{*} \varphi} t^{N s} \frac{d t}{t} .
$$

Now, as in the the proof of Proposition 17, the Poisson summation formula implies that the right hand side is absolutely convergent for $\operatorname{Re}(s)$ sufficiently large and extends to a meromorphic function of $s$ which is holomorphic at $s=0$ equal to $-\widehat{\varphi}(0)$. Using the explicit formula for $\varphi$ (see Theorems 12 and 13) we conclude that the form $E_{\psi}(g, v ; 0)$ is a primitive of $-\mathrm{vol}_{N}$.

To prove (8.10) we have to take into account the behavior near the lattice points.

\section{The EISENSTEIn TRANSGRESSION FOR $\mathrm{GL}_{2}(\mathbf{R})$ AND CLASSICAL MODUlaR FORMS}

In the case $N=2$, we have $X=\mathcal{H}$ and the universal family of tori is the universal elliptic curve. In this section we give explicit formulas for the form $E_{\psi}$ and relate it to classical modular forms, compare with [4].

9.1. The universal elliptic curve. Given $\tau \in \mathcal{H}$ we denote by $\Lambda_{\tau}$ the lattice $\mathbf{Z}+\mathbf{Z} \tau$ in $\mathbf{C}$ and let $\mathcal{E}_{\tau}=\mathbf{C} / \Lambda_{\tau}$ be the associated elliptic curve. The action of $\mathrm{SL}_{2}(\mathbf{Z})$ on $\mathcal{H} \times \mathbf{C}$ preserves the subset $\left\{(\tau, z): z \in \Lambda_{\tau}\right\}$. Now $\mathbf{Z}^{2}$ acts on $\mathcal{H} \times \mathbf{C}$ by

$$
(u, v) \cdot(\tau, z)=(\tau, z+u-v \tau)
$$

and $\mathrm{SL}_{2}(\mathbf{Z})$ on the quotient. The resulting double quotient

$$
\mathrm{SL}_{2}(\mathbf{Z}) \backslash\left((\mathcal{H} \times \mathbf{C}) / \mathbf{Z}^{2}\right)
$$

is called the universal elliptic curve.

9.2. The Eisenstein series $E_{\psi}$. The Eisenstein series $E_{\psi}$ defines a differential form on the universal elliptic curve. On $\mathcal{H} \times \mathbf{C}$ the 1 -form $d z$ is not invariant under the $\mathbf{Z}^{2}$-action but the form

$$
\alpha=d z-\frac{z-\bar{z}}{2 i} \frac{d \tau}{y}
$$

is; in the coordinates $z=u-v \tau$, we have: $\alpha=d u-\tau d v$.

We will derive an explicit expression for the 1-form $E_{\psi}$ in the basis of 1-forms given by $\alpha, \beta=d \tau$ and their complex conjugates. These forms are invariant under the $\mathbf{Z}^{2}$-action and it follows from (7.6) that we have:

$$
\psi=\frac{1}{2 \pi}\left(\frac{|z|^{2}}{2 y} e^{-\frac{|z|^{2}}{2 y}}\right)\left[i\left(\frac{\alpha}{z}-\frac{\bar{\alpha}}{\bar{z}}\right)-\frac{1}{2 y}\left(\frac{\bar{z}}{z} \beta+\frac{z}{\bar{z}} \bar{\beta}\right)\right] .
$$


Now consider the 1 -form

$$
\begin{aligned}
\theta_{t^{*} \psi}^{*}(\tau, z)=\operatorname{Re}\left(-\frac{1}{2 \pi y} \sum_{\omega \in \mathbf{Z}+\mathbf{Z} \tau}^{\prime} \frac{t^{2}|z+\omega|^{2}}{2 y} e^{-\frac{t^{2}|z+\omega|^{2}}{2 y}} \frac{\bar{z}+\bar{\omega}}{z+\omega} \beta\right. \\
\left.+\frac{i}{\pi} \sum_{\omega \in \mathbf{Z}+\mathbf{Z} \tau}^{\prime} \frac{t^{2}|z+\omega|^{2}}{2 y} e^{-\frac{t^{2}|z+\omega|^{2}}{2 y}} \frac{\alpha}{z+\omega}\right),
\end{aligned}
$$

where $\sum^{\prime}$ means that if $z \in \mathbf{Z}+\mathbf{Z} \tau$ we omit the term corresponding to $\omega=-z$. The Mellin transform of $\theta_{t^{*} \psi}^{*}(\tau, z)$ gives

$$
\begin{aligned}
E_{\psi}(\tau, z ; s)= & \int_{0}^{\infty} \theta_{t^{*} \psi}^{*}(\tau, z) t^{2 s} \frac{d t}{t} \\
= & \operatorname{Re}\left[2 ^ { s - 1 } \Gamma ( s + 1 ) y ^ { s } \left(-\frac{1}{2 \pi y} \sum_{\omega \in \mathbf{Z}+\mathbf{Z} \tau}^{\prime} \frac{(\bar{z}+\bar{\omega})^{2}}{|z+\omega|^{2 s+2}} \beta\right.\right. \\
& \left.\left.+\frac{i}{\pi} \sum_{\omega \in \mathbf{Z}+\mathbf{Z} \tau}^{\prime} \frac{\bar{z}+\bar{\omega}}{|z+\omega|^{2 s+2}} \alpha\right)\right] .
\end{aligned}
$$

In conclusion, the Eisenstein series $E_{\psi}(\tau, z ; s)$ is the real part of the sum

(9.4) $-2^{s-2} \Gamma(s+1) \frac{y^{s-1}}{\pi} K_{2}(s+1, \tau, z, 0) \beta+i 2^{s-1} \Gamma(s+1) \frac{y^{s}}{\pi} K_{1}(s+1, \tau, z, 0) \alpha$,

where $K_{a}(s, \tau, z, u)$ is the classical Eisenstein-Kronecker series of weight $a$ (see [50, Eq. (27) p. 78]). In the next paragraph we briefly recall some of the properties of these series. Note that (9.4) is $\mathbf{Z}^{2}$-invariant and therefore defines a 1 -form on $(\mathcal{H} \times \mathbf{C}) / \mathbf{Z}^{2}$.

9.3. The Eisenstein-Kronecker series. Given $\tau \in \mathcal{H}$ and $z, u \in \mathbf{C}$, the EisensteinKronecker series is defined by

$$
K_{a}(s, \tau, z, u)=\sum_{\omega \in \mathbf{Z}+\mathbf{Z} \tau}^{\prime} \frac{(\bar{\omega}+\bar{z})^{a}}{|\omega+z|^{2 s}} e\left(\frac{\omega \bar{u}-u \bar{\omega}}{\tau-\bar{\tau}}\right) .
$$

Here $a$ is some integer, $e(z)=e^{2 i \pi z}$, and the sum is absolutely convergent for $\operatorname{Re}(s)>$ $\frac{a}{2}+1$.

The Eisenstein-Kronecker series $K_{a}$ satisfies a functional equation: let

$$
K_{a}^{*}(s, \tau, z, u)=\pi^{-s} \Gamma(s) y^{s-a} K_{a}(s, \tau, z, u) .
$$

It has meromorphic continuation to the whole complex $s$-plane with simple poles in $s=1$ (if $a=0$ and $u \in \mathbf{Z}+\mathbf{Z} \tau$ ) and $s=0$ (if $a=0$ and $z \in \mathbf{Z}+\mathbf{Z} \tau$ ), and we have

$$
K_{a}^{*}(s, \tau, z, u)=e\left(\frac{z \bar{u}-u \bar{z}}{\tau-\bar{\tau}}\right) K_{a}^{*}(a+1-s, \tau, u, z) .
$$

(See [50, Eq. (32) p. 80].) In particular our Eisenstein series (9.4) is absolutely convergent for $\operatorname{Re}(s)>1$ with meromorphic continuation to the whole complex $s$-plane.

We are interested in the value at $s=0$. We define

$$
E_{1}(\tau, z)=\frac{i}{2 \pi} K_{1}(1, \tau, z, 0)
$$

It is a (non-holomorphic) way to make sense of the divergent series

$$
\frac{i}{2 \pi} \sum_{\omega \in \mathbf{Z}+\mathbf{Z} \tau}^{\prime} \frac{1}{\omega+z} .
$$


We similarly define

$$
\begin{aligned}
E_{2}(\tau, z) & =-\frac{1}{4 \pi y} K_{2}(1, \tau, z, 0) \\
& =-\frac{1}{2 \pi^{2}} K_{2}(2, \tau, 0, z) .
\end{aligned}
$$

Here the equality follows from the functional equation (9.5). It makes sense of the formal series

$$
-\frac{1}{2 \pi^{2}} \sum_{\omega \in \mathbf{Z}+\mathbf{Z} \tau}^{\prime} \frac{1}{\omega^{2}} e\left(\frac{\omega \bar{z}-z \bar{\omega}}{\tau-\bar{\tau}}\right) .
$$

It follows from (9.4) that the 1 -form $E_{\psi}$ is the real part of

$$
E_{1}(\tau, z) \alpha+E_{2}(\tau, z) \beta .
$$

9.4. Further transgression. Let $\mathcal{L} \rightarrow \mathcal{H}$ be the Hodge line bundle on $\mathcal{H}$. Then $\mathcal{L}$ carries a canonical hermitian metric $h$ and we have an isomorphism of metrized bundles $\mathcal{L} \cong \mathcal{H} \times \mathbf{C}$, where the latter is equipped with the metric $y^{-1}|\cdot|^{2}$ on the fiber over $\tau \in \mathcal{H}$. In other words, in this case the basic geometric setup is the Hodge line bundle $(\mathcal{L}, h) \rightarrow \mathcal{H}$, a holomorphic line bundle over the complex manifold $\mathcal{H}$. In this setting, Bismut-GilletSoulé [7, Theorem 1.15] have refined the canonical transgression form $\psi$ to a canonical $\partial \bar{\partial}$-transgression. Namely, there is a smooth function

$$
\nu \in A^{0}(\mathcal{L}),
$$

rapidly decreasing along the fibers of $\mathcal{L}$, such that

$$
\partial \bar{\partial} \nu(t v)=t \frac{d}{d t} \varphi(t v)
$$

(compare Equation (7.4)). In the coordinates of $\$ 7.4$ we have

$$
\nu=\frac{1}{2 \pi} e^{-|z|^{2} / 2 y} .
$$

The form satisfies

$$
i(\partial-\bar{\partial}) \nu=\psi
$$

To globalize this double transgression one can replace $\psi$ by $\nu$ in the computations above. We get:

$$
\begin{aligned}
E_{\nu}(\tau, z ; s) & =\int_{0}^{\infty} \theta_{t^{*} \nu}(\tau, z) t^{2 s} \frac{d t}{t} \\
& =\frac{1}{\pi} 2^{s-2} y^{s} \sum_{\omega \in \mathbf{Z}+\mathbf{Z} \tau}^{\prime} \frac{1}{|z+\omega|^{2 s}} \\
& =\frac{1}{\pi} 2^{s-2} y^{s} K_{0}(s, \tau, z, 0) .
\end{aligned}
$$

Letting

$$
E_{\nu}=E_{\nu}(\tau, z ; 0) \in A^{0}(\mathcal{L})^{\mathbf{Z}^{2}}
$$

we deduce from (9.5) that

$$
E_{\nu}=\frac{y}{4 \pi^{2}} K_{0}(1, \tau, 0, z) .
$$

It is a way to make sense of the sum of the (non absolutely convergent) series

$$
\frac{y}{4 \pi^{2}} \sum_{m, n \in \mathbf{Z}}^{\prime} \frac{e^{2 i \pi(m u+n v)}}{|m+n \tau|^{2}},
$$


where $z=v-u \tau$. It finally follows from Kronecker second limit formula [47, Eqn. (39) p. 28] that

$$
E_{\nu}=\frac{1}{2 y}(\operatorname{Im} z)^{2}-\frac{1}{2 \pi} \log |\theta(\tau, z)|
$$

where $\theta$ is the classical Jacobi theta function, defined by the infinite product

$$
\theta(\tau, z)=q^{1 / 12}\left(q_{z}^{1 / 2}-q_{z}^{-1 / 2}\right) \prod_{n \geq 1}\left(\left(1-q^{n} q_{z}\right)\left(1-q^{n} q_{z}^{-1}\right)\right)
$$

$\left(q=e(\tau)\right.$ and $\left.q_{z}=e(z)\right)$. We have $E_{\psi}=i(\partial-\bar{\partial}) E_{\nu}$ and one can then check directly that on $\mathcal{H} \times \mathbf{C}$, away from the zero section, we have

$$
\begin{aligned}
d E_{\psi} & =2 i \partial \bar{\partial} E_{\nu} \\
& =2 i \partial \bar{\partial}\left(\frac{1}{2 y}(\operatorname{Im} z)^{2}\right) \\
& =-\frac{i}{2 y} \alpha \wedge \bar{\alpha}
\end{aligned}
$$

where the 2-form on the right hand side is $\mathrm{SL}_{2}(\mathbf{Z}) \ltimes \mathbf{Z}^{2}$-invariant and restricts to the standard normalized volume form $-\frac{i d z \wedge d \bar{z}}{2 y}$ on each fiber $\mathbf{C} /(\mathbf{Z}+\tau \mathbf{Z})$.

\section{RELATION BETWEen the EISENSTEIN TRANSGRESSiON AND the EISENSTEIN}

\section{CLASS}

In this section we finally relate the $(N-1)$-form $E_{\psi}$ on $T-\{0\}$ with the classes $z_{m}$ of Definition 10.

10.1. Action of $a_{*}$ on $E_{\psi}$. By (8.6) we may think of $E_{\psi}$ as a (regularized) push-forward of a form $\theta_{\varphi}$ on the fiber bundle

$$
\Gamma \backslash\left(S^{+} \times(V-L) / L\right) \rightarrow T-\{0\}
$$

with fiber $\mathbf{R}_{>0}$. The following proposition should therefore not be surprising.

Proposition 20. Let a be a positive integer. Under the action of the push forward map $a_{*}$ induced by multiplication on the fibers $a: T-T[a] \rightarrow T-\{0\}$, the restriction of the form $E_{\psi}$ to $T-T[a]$ is mapped to $E_{\psi}$ :

$$
a_{*}\left(E_{\psi}\right)=E_{\psi} .
$$

Proof. By definition of the push forward map, we have:

$$
\left[a_{*}\left(\theta_{\psi}\right)\right](g, v)=\sum_{w \in a^{-1}(v+L) / L} \theta_{\psi}(g, w) .
$$

Now the right hand side is equal to

$$
\sum_{w \in a^{-1}(v+L) / L} \sum_{\lambda \in L} \psi\left(g^{-1}(\lambda-w)\right)=\sum_{\lambda \in L} \psi\left(g^{-1} a^{-1}(\lambda-v)\right)=\theta_{\psi}(a g, v) .
$$

It follows that

$$
\begin{aligned}
a_{*} E_{\psi}(g, v ; s) & =\int_{0}^{+\infty} a_{*} \theta_{\psi}\left(t^{-1} g, v\right) t^{s} \frac{d t}{t} \\
& =\int_{0}^{+\infty} \theta_{\psi}\left(a t^{-1} g, v\right) t^{s} \frac{d t}{t} \\
& =a^{s} E_{\psi}(g, v ; s) .
\end{aligned}
$$


Taking $s=0$ we get the proposition.

10.2. A de Rham realization of $z_{m}$. We can now prove Theorem 3 of the Introduction. Recall that in Definition 10 we have introduced a rational cohomology class

$$
z_{m} \in H^{N-1}(T-T[m], \mathbf{Q}) .
$$

Theorem 21. The expression

$$
E_{\psi}^{(m)}(g, v):=E_{\psi}(g, m v)-m^{N} E_{\psi}(g, v)
$$

defines a closed $(N-1)$-form on $T-T[m]$ whose cohomology class in $H^{N-1}(T-$ $T[m], \mathbf{R}$ ) is equal to (the image of) $z_{m}$.

Proof. By Theorem 19, the $(N-1)$-form $E_{\psi}^{(m)}$ is closed on $T-T[m]$ and its cohomology class in $H^{N-1}(T-T[m])$ is mapped onto the class

$$
\left[T[m]-m^{N}\{0\}\right] \in H^{N}(T, T-T[m])
$$

in the long exact sequence in cohomology associated to the pair $(T, T[m])$.

Proposition 20 furthermore implies that if $a$ is an integer coprime to $m$, the differential form $E_{\psi}^{(m)}$ is fixed by $a_{*}$. Since $z_{m}$ is the unique such lift of (10.1), this concludes the proof of the theorem.

Example. Getting back to the case of $\mathrm{GL}_{2}(\mathbf{R})$, we note that even though the Jacobi theta function $\theta(\tau, z)$ is not $(\mathbf{Z}+\mathbf{Z} \tau)$-periodic in $z$, if $m>2$ is an integer coprime to 6 , the function

$$
g_{0, m}=\theta(\tau, z)^{m^{2}} \theta(\tau, m z)^{-1}
$$

is. It follows from the computations of $\S 9.4$ that

$$
E_{\psi}^{(m)}=\frac{i}{2 \pi}(\partial-\bar{\partial}) \log \left|g_{0, m}\right|,
$$

and indeed the divisor on the elliptic curve $\mathcal{E}_{\tau}=\mathbf{C} /(\mathbf{Z}+\mathbf{Z} \tau)$ associated to $g_{0, m}$ is $\mathcal{E}_{\tau}[m]-$ $m^{2}\{0\}$.

10.3. Pull-back by a torsion section. Now let $v$ be a rational vector in $V$ that does not belong to $L$. Suppose that the class of $v$ modulo $L$ is fixed by $\Gamma$. It corresponds to $v$ a (torsion) section

$$
\mathbf{v}: \Gamma \backslash X \rightarrow T-\{0\} .
$$

Definition 22. We denote by

$$
E_{\psi, v}=\mathbf{v}^{*} E_{\psi}
$$

the pull-back of the differential form $E_{\psi}$ by the torsion section $\mathbf{v}$.

As a function of $g$ we have

$$
E_{\psi, v}(g)=E_{\psi}(g, v) .
$$

Theorem 19 implies that $E_{\psi, v}$ is a closed $(N-1)$-form on $\Gamma \backslash X$; it therefore defines a cohomology class

$$
\left[E_{\psi, v}\right] \in H^{N-1}(\Gamma \backslash X) .
$$

Note that, for all $m$ we have

$$
\mathbf{v}^{*} E_{\psi}^{(m)}=E_{\psi, m v}-m^{N} E_{\psi, v} .
$$


Now recall that, in Definition 11, we have defined a natural rational cohomology class

$$
z(\mathbf{v}) \in H^{N-1}(\Gamma \backslash X, \mathbf{Q})
$$

associated to the torsion section $\mathbf{v}$.

Theorem 23. Assume that $v$ is of order coprime to $m$ and that $m v=v$, then we have

$$
\left[E_{\psi, v}\right]=-z(\mathbf{v}) \text { in } H^{N-1}(\Gamma \backslash X) .
$$

Proof. By definition of $z(\mathbf{v})$, it follows from Theorem 21 that

$$
z(\mathbf{v})=\frac{1}{m^{N}-1} \mathbf{v}^{*}\left(z_{m}\right)=\frac{1}{m^{N}-1} \mathbf{v}^{*}\left[E_{\psi}^{(m)}\right] .
$$

Now, since $m v=v$, the cohomology class of the pullback of $(g, v) \mapsto E_{\psi}(g, m v)$ by $\mathbf{v}^{*}$ is equal to the cohomology class of $E_{\psi, v}$ and we conclude that

$$
z(\mathbf{v})=\frac{1}{m^{N}-1}\left(\left[E_{\psi, v}\right]-m^{N}\left[E_{\psi, v}\right]\right)=-\left[E_{\psi, v}\right] .
$$

Remark. Using the remark following Definition 11, one shows that the class $\left(m^{N}-1\right) z(\mathbf{v})$ in fact belongs to $H^{N-1}\left(\Gamma \backslash X, \mathbf{Z}_{\ell}\right)$ for any $\ell$ coprime to $m$. As a consequence, the class $\left[E_{\psi, v}\right]$ is 'almost integral:'

$$
d_{N}\left[E_{\psi, v}\right] \in H^{N-1}(\Gamma \backslash X, \mathbf{Z}) .
$$

Here $d_{N}$ is the denominator of half the $N$-th Bernoulli number. Note that Bismut and Cheeger also prove rationality statements for the general cohomology class they define, see $[6,8]$.

\section{More $\mathrm{SL}_{2}(\mathbf{R})$ COMputations : Dedekind-RAdemacher And DAMERell RESULTS}

Let us come back to the setting of Section 9 where $E_{\psi, v}$ defines a 1-form on a congruence cover $\Gamma \backslash \mathcal{H}$ of the modular surface. In this section, we relate this 1 -form to classical modular functions. We however want to emphasis that one should refrain to pull back $E_{\psi}$ by a torsion section too hastily. To motivate this last sentence, in the last paragraph of this section we explain how to derive a classical theorem of Damerell [20] by considering the 1 -form $E_{\psi}^{(m)}$ on the total space of the universal elliptic curve.

11.1. The 1-forms $E_{\alpha, \beta}$. Let $(\alpha, \beta) \in(\mathbf{Q} / \mathbf{Z})^{2}$ and let $(k / \ell, r / \ell) \in \mathbf{Q}^{2}$ be a representative. If $\Gamma$ is a congruence subgroup of $\mathrm{SL}_{2}(\mathbf{Z})$ that fixes $(\alpha, \beta)$ in $(\mathbf{Q} / \mathbf{Z})^{2}$, the map

$$
\mathcal{H} \rightarrow \mathcal{H} \times \mathbf{C} ; \tau \mapsto(\tau,(k-r \tau) / \ell))
$$

induces a section

$$
s_{\alpha, \beta}: \Gamma \backslash \mathcal{H} \rightarrow \Gamma \backslash\left((\mathcal{H} \times \mathbf{C}) / \mathbf{Z}^{2}\right) .
$$

Now the Eisenstein series (9.4) defines a $\Gamma$-invariant 1 -form on the universal elliptic curve with level structure $\Gamma \backslash\left((\mathcal{H} \times \mathbf{C}) / \mathbf{Z}^{2}\right)$, and it follows from (9.6) that its pull-back by the section $s_{\alpha, \beta}$ is the 1 -form

$$
\left.\left.E_{\alpha, \beta}=\operatorname{Re}\left(E_{2}(\tau,(k-r \tau) / \ell)\right) d \tau\right)=-\frac{1}{2 \pi^{2}} \operatorname{Re}\left(K_{2}(2, \tau, 0,(k-r \tau) / \ell)\right) d \tau\right)
$$

on $\Gamma \backslash \mathcal{H}$. 
11.2. Some classical facts on the weight 2 Eisenstein-Kronecker series. As we have seen, the series

$$
\left.K_{2}(s+2, \tau, 0,(k-r \tau) / \ell)\right)=\sum_{m, n}^{\prime} \frac{e\left(\frac{m k+n r}{\ell}\right)}{(m \tau+n)^{2}|m \tau+n|^{2 s}}
$$

is absolutely convergent for $\operatorname{Re}(s)>0$, and for fixed $\tau$ can be extended by analytic continuation to a function of $s$ on the entire complex plane. Defining

$$
\left.E_{2,(\alpha, \beta)}(\tau):=K_{2}(2, \tau, 0,(k-r \tau) / \ell)\right),
$$

the regularization procedure we have used so far is Hecke's way of making sense of the formal sum

$$
\sum_{m, n} \frac{e\left(\frac{m k+n r}{\ell}\right)}{(m \tau+n)^{2}}
$$

Another way - due to Eisenstein - is to specify an order of summation for the terms in the conditionally convergent sum. We may define

$$
E_{2,(\alpha, \beta)}^{*}(\tau):=\sum_{m} \sum_{n} \frac{e\left(\frac{m k+n r}{\ell}\right)}{(m \tau+n)^{2}}
$$

where $m, n$ run over $\mathbf{Z}^{2}$ in the prescribed order, omitting $(0,0)$. The Hecke summation and the Eisenstein summation yield the same function when $(\alpha, \beta) \neq(0,0) \in(\mathbf{Q} / \mathbf{Z})^{2}$. However in general we have:

$$
E_{2,(\alpha, \beta)}(\tau)= \begin{cases}E_{2,(\alpha, \beta)}^{*}(\tau), & \text { if }(\alpha, \beta) \neq(0,0) \in(\mathbf{Q} / \mathbf{Z})^{2} \\ E_{2,(0,0)}^{*}(\tau)-\frac{\pi}{\operatorname{Im}(\tau)}, & \text { if }(\alpha, \beta)=(0,0) \in(\mathbf{Q} / \mathbf{Z})^{2}\end{cases}
$$

see e.g. [50, Part III, Eqn. (11)].

The holomorphic function $E_{2,(0,0)}^{*}(\tau)$ on $\mathcal{H}$ is not a modular form of weight 2 . On the other hand $E_{2,(0,0)}(\tau)=E_{2,(0,0)}^{*}(\tau)-\frac{\pi}{\operatorname{Im}(\tau)}$ is modular but not holomorphic. We will mainly consider $E_{2,(\alpha, \beta)}(\tau)$ with $(\alpha, \beta) \neq(0,0) \in(\mathbf{Q} / \mathbf{Z})^{2}$. It defines a modular form of weight 2 for the group $\Gamma(\ell)$.

Remark. We recover in particular that our real 1-forms

$$
E_{\alpha, \beta}=-\frac{1}{2 \pi^{2}} \operatorname{Re}\left(E_{2,(\alpha, \beta)}(\tau) d \tau\right)
$$

are closed except when $(\alpha, \beta)=(0,0) \in(\mathbf{Q} / \mathbf{Z})^{2}$. In the latter case we get a transgression of the Euler/area form:

$$
d E_{0,0}=\frac{1}{2 \pi} \frac{d x \wedge d y}{y^{2}}
$$

11.3. Dedekind-Rademacher homomorphisms. One can attach to the form $E_{\alpha, \beta}$ a homomorphism $\Phi_{\alpha, \beta}: \Gamma \rightarrow \mathbf{Q}$ defined by the rule

$$
\Phi_{\alpha, \beta}(\gamma)=-\int_{\tau_{0}}^{\gamma \tau_{0}} E_{\alpha, \beta},
$$

for any $\tau_{0} \in \mathcal{H}$. The fact that $E_{\alpha, \beta}$ is a closed 1-form implies that the value (11.2) is independent of $\tau_{0}$ and of the path from $\tau_{0}$ to $\gamma \tau_{0}$. The fact that $\Phi_{\alpha, \beta}(\gamma)$ is a rational 
number follows from the fact that $E_{\alpha, \beta}$ defines a rational cohomology class. We deduce from [47, Theorem 13] that

$$
\Phi_{\alpha, \beta}\left(\begin{array}{ll}
a & b \\
c & d
\end{array}\right)= \begin{cases}\widetilde{B}_{2}(\beta) \frac{b}{d} & \text { if } c=0 \\
\widetilde{B}_{2}(\beta) \frac{a+d}{c}-2 \operatorname{sgn}(c) s_{\alpha, \beta}(a /|c|) & \text { otherwise. }\end{cases}
$$

Here

$$
\widetilde{B}_{2}(x)=B_{2}(x-[x])=\frac{1}{2 \pi^{2}} \sum_{n \in \mathbf{Z}}^{\prime} \frac{e(n x)}{n^{2}}
$$

is the 'periodic second Bernoulli polynomial' with $B_{2}(X)=X^{2}-X+\frac{1}{6}$, and $s_{\alpha, \beta}$ is the generalized Dedekind sum

$$
s_{\alpha, \beta}(r / m)=\sum_{k=0}^{m-1} \widetilde{B}_{1}\left(\frac{k-\beta}{m}\right) \widetilde{B}_{1}\left(r \frac{k-\beta}{m}-\alpha\right),
$$

for $m>0$ and $(r, m)=1$. Here $\widetilde{B}_{1}: \mathbf{Q} \rightarrow \mathbf{Q}$ is the 'periodic first Bernoulli polynomial' $x \mapsto x-[x]-1 / 2$.

A formula of Siegel [47] relates $\Phi_{\alpha, \beta}(\gamma)$ to some special values of $L$-functions. For general $N$, this is systematically explored in the next section where we adopt the more convenient adelic formulation.

11.4. A theorem of Damerell. To conclude this section, concerned with the special case $N=2$, we want to put the accent on the importance of considering the closed form

$$
E_{\psi}(\tau, z)^{(m)}=E_{\psi}(\tau, m z)-m^{2} E_{\psi}(\tau, z),
$$

that gives the de Rham realization of the Eisenstein class in $H^{1}(T-T[m], \mathbf{Q})$ on the total space $T-T[m]$, and not only its pullback by a torsion section.

Consider indeed the pullback of $E_{\psi}^{(m)}$ to a fixed CM elliptic curve $\mathcal{E}_{\tau}=\mathbb{C} / \Lambda_{\tau}$. It follows from (9.4) that

$$
\left.E_{\psi}(\tau, z ; s)\right|_{\mathcal{E}_{\tau}}=\frac{i}{4 \pi} \Gamma(s+1)(2 y)^{s}\left(K_{1}(s+1, \tau, z, 0) d z-\overline{K_{1}(s+1, \tau, z, 0)} d \bar{z}\right) .
$$

We may then write

$$
E_{\psi}(\tau, z)^{(m)}=\omega^{1,0}+\omega^{0,1},
$$

where $\omega^{1,0}$ is a holomorphic 1 -form and $\omega^{0,1}=\overline{\omega^{1,0}}$. Moreover: $\omega^{1,0}$ has residue 1 at each nonzero torsion point in $\mathcal{E}_{\tau}[m]$ and residue $\left(1-m^{2}\right)$ at $0 \in \mathcal{E}_{\tau}$. Now the RiemannRoch Theorem implies that one can find a meromorphic function $f$ on $\mathcal{E}_{\tau}$ with divisor $\mathcal{E}_{\tau}[m]-m^{2}\{0\}$ - we may, and will, moreover choose $f$ to be defined over $\overline{\mathbf{Q}}$. Hence the difference

$$
\omega^{1,0}-(2 \pi i)^{-1} d f / f
$$

extends to a holomorphic differential on $\mathcal{E}_{\tau}$, so that

$$
\omega^{1,0}=(2 \pi i)^{-1} d f / f+C d z
$$

for some complex number $C$. Now one can show that $C=0$ by looking at the operator $a_{*}$ for an integer $a$ coprime to $m$ : we have $a_{*} d z=a d z$, however $a_{*}(d f / f)=d f / f$ and $a_{*} \omega^{1,0}=\omega^{1,0}$.

Assume that $\mathcal{E}_{\tau}$ has complex multiplication by an order $\mathcal{O}$ in an imaginary quadratic field $K$, let $\omega_{\text {alg }}$ be a holomorphic global differential on $\mathcal{E}_{\tau}$ defined over $\overline{\mathbf{Q}}$ and let $\Omega \in$ $\mathbf{R}_{>0}$ be such that the rational periods of $\omega_{\text {alg }}$ are $K \cdot \Omega \subset \mathbf{C}$. Identifying $\Lambda_{\tau}$ with $\Omega \mathcal{O}$ we 
see that we can take $\omega_{\text {alg }}=\Omega d z$. Now let $P$ be a torsion point that is not $m$-torsion and evaluate both sides of

$$
2 \pi i \omega^{1,0}=d f / f
$$

at $P$. Since we have taken $f$ to be algebraic, the right hand side of (11.7) belongs to $\overline{\mathbf{Q}} \cdot \omega_{\mathrm{alg}}(P)$. The left hand side of (11.7) equals

$$
-\frac{1}{2} K_{1}(1, \tau, z, 0) \Omega^{-1} \cdot(\Omega d z)
$$

and we conclude the following:

Theorem 24 (Damerell). We have:

$$
K_{1}(1, \tau, z, 0) \in \overline{\mathbf{Q}} \Omega .
$$

\section{Adelic formulation, Period COMPutations And the KLINGEn-Siegel THEOREM}

In this section we come back to our general setting and treat

$$
\psi \in\left[A^{N-1}\left(\mathrm{GL}_{N}(\mathbf{R}) / \mathrm{SO}_{N}\right) \otimes \mathcal{S}(V)\right]^{\mathrm{GL}_{N}(\mathbf{R})}
$$

as the Archimedean component of a global Schwartz form to which we apply the theta operator in adelic formulation. The explicit definition of this Archimedean component is a key feature of our invariant viewpoint while in the theory of automorphic forms it is usually hard to pin down a good choice of Archimedean vector.

To exemplify the advantage of using $\psi$ we revisit the classical computation of toric periods of degenerate Eisenstein series. It is known that these periods are special values of partial $L$-functions of (finite order) Hecke characters, see [51]. Here we show that the periods of the Eisenstein form $E_{\psi}$ are special values of the corresponding global $L$ functions. The cohomology class $E_{\psi}$ being rational we immediately recover the KlingenSiegel Theorem.

12.1. The adelic quotient. Let $\mathbf{A}$ be the adÃíle ring of $\mathbf{Q}$ and let

$$
\left[\mathrm{GL}_{N}\right]=\mathrm{GL}_{N}(\mathbf{Q}) \backslash \mathrm{GL}_{N}(\mathbf{A}) / \mathrm{SO}_{N} Z(\mathbf{R})^{+} .
$$

Strong approximation for $\mathrm{GL}_{N}$ implies that, for any compact open subgroup $K \subset \mathrm{GL}_{N}\left(\mathbf{A}_{f}\right)=$ $\mathrm{GL}_{N}(\widehat{\mathbf{Z}})$, the double quotient

$$
Z\left(\mathbf{A}_{f}\right) \backslash\left[\mathrm{GL}_{N}\right] / K=\mathrm{GL}_{N}(\mathbf{Q}) \backslash\left(\left(\mathrm{GL}_{N}(\mathbf{R}) / \mathrm{SO}_{N} Z(\mathbf{R})^{+}\right) \times \mathrm{GL}_{N}\left(\mathbf{A}_{f}\right)\right) / K
$$

is a finite union of finite volume quotients of $X$ that can be described as follows. Write

$$
\mathrm{GL}_{N}\left(\mathbf{A}_{f}\right)=\coprod_{j} \mathrm{GL}_{N}(\mathbf{Q})^{+} g_{j} K \text {. }
$$

Here $\mathrm{GL}_{N}(\mathbf{Q})^{+}=\mathrm{GL}_{N}(\mathbf{Q}) \cap \mathrm{GL}_{N}(\mathbf{R})^{+}$. Then

$$
Z(\mathbf{A}) \backslash\left[\mathrm{GL}_{N}\right] / K=\coprod_{j} \Gamma_{j} \backslash X .
$$

Here $\Gamma_{j}$ is the image of $\mathrm{GL}_{N}(\mathbf{Q})^{+} \cap g_{j} K g_{j}^{-1}$ in $\mathrm{GL}_{N}(\mathbf{R})^{+} / Z(\mathbf{R})^{+}$. 


\subsection{Adelic theta series. Define a map}

$$
\theta_{\psi}^{*}: \mathcal{S}\left(V\left(\mathbf{A}_{f}\right)\right) \rightarrow A^{N-1}\left(\mathrm{GL}_{N}(\mathbf{Q}) \backslash \mathrm{GL}_{N}\left(\mathbf{A}_{f}\right) \times \mathrm{GL}_{N}(\mathbf{R}) / \mathrm{SO}(N)\right)
$$

by

$$
\theta_{\psi}^{*}\left(g_{f} ; \varphi_{f}\right)=\sum_{v \in V(\mathbf{Q})-\{0\}} \omega\left(g_{f}\right) \varphi_{f}(v) \psi(v)
$$

Note that the left invariance under $\mathrm{GL}_{N}(\mathbf{Q})$ means that $\gamma^{*} \theta^{*}\left(\gamma g_{f} ; \varphi_{f}\right)=\theta^{*}\left(g_{f}, \varphi_{f}\right)$ for any $\gamma \in \mathrm{GL}_{N}(\mathbf{Q})$; this follows from the $\mathrm{GL}_{N}(\mathbf{R})$-invariance of $\psi$, see (8.1). The map $\theta^{*}$ intertwines the natural action of $\mathrm{GL}_{N}\left(\mathbf{A}_{f}\right)$ on both sides.

12.3. Adelic Eisenstein series. Note that for $-1 \in Z(\mathbf{R})$ we have

$$
(-1)^{*} \psi(v)=\psi(-v)=(-1)^{N} \psi(v) .
$$

Let $\omega=\otimes_{v} \omega_{v}: \mathbf{Q}^{\times} \backslash \mathbf{A}^{\times} \rightarrow \mathbf{C}^{\times}$be a character of finite order such that $\omega_{\infty}(x)=$ $\operatorname{sgn}(x)^{N}$ and let $K_{\omega}=\operatorname{ker}\left(\left.\omega\right|_{\hat{\mathbf{z}}^{\times}}\right)$. For $\varphi_{f} \in \mathcal{S}\left(V\left(\mathbf{A}_{f}\right)\right), g_{f} \in \mathrm{GL}_{N}\left(\mathbf{A}_{f}\right)$ and $\operatorname{Re}(s) \gg 1$, we define

$$
E_{\psi}\left(g_{f}, \omega, \varphi_{f}, s\right)=\int_{\mathbf{Q}^{\times} \backslash \mathbf{A} \times / K_{\omega}} z_{\infty}^{*} \theta_{\psi}^{*}\left(z_{f} g_{f} ; \varphi_{f}\right) \omega(z)|z|^{N s} \frac{d z_{\infty}}{z_{\infty}} .
$$

Then

$$
E_{\psi}\left(\omega, \varphi_{f}, s\right) \in A^{N-1}\left(\mathrm{GL}_{N}(\mathbf{Q}) \backslash \mathrm{GL}_{N}(\mathbf{A}) / \mathrm{SO}(N)\right)
$$

and we have

$$
z_{\infty}^{*} E_{\psi}\left(z_{f} g_{f}, \omega, \varphi_{f}, s\right)=\omega(z)^{-1}|z|^{-N s} E_{\psi}\left(g_{f}, \omega, \varphi_{f}, s\right), \quad z=\left(z_{f}, z_{\infty}\right) \in \mathbf{A}^{\times} .
$$

Using Poisson summation, as in the preceding section, one proves that $E_{\psi}$ admits meromorphic continuation to $s \in \mathbf{C}$ with at most simple poles at $s=0$ and $s=1$. Moreover, there is no pole at $s=0$ if $\varphi_{f}(0)=\widehat{\varphi}_{f}(0)=0$. In that case we set

$$
E_{\psi}\left(\omega, \varphi_{f}\right)=\left.E_{\psi}\left(\omega, \varphi_{f}, s\right)\right|_{s=0} \in A^{N-1}\left(\left[\mathrm{GL}_{N}\right]\right) .
$$

By Theorem 19, the form $E_{\psi}\left(\omega, \varphi_{f}\right)$ is closed. We obtain a cohomology class

$$
\left[E_{\psi}(\omega)\right] \in \mathrm{H}^{N-1}\left(\left[\mathrm{GL}_{N}\right], \mathcal{S}_{0}\left(V\left(\mathbf{A}_{f}\right)\right)^{\vee}\right)
$$

with central character $\omega_{f}^{-1}$. Here $\mathcal{S}_{0}\left(V\left(\mathbf{A}_{f}\right)\right)$ denotes the subspace of $\mathcal{S}\left(V\left(\mathbf{A}_{f}\right)\right)$ defined by $\varphi_{f}(0)=\widehat{\varphi}_{f}(0)=0$.

12.4. Homology classes. There are natural degree $N-1$ homology classes on which to test the Eisenstein class: let $\gamma \in \mathrm{GL}_{N}(\mathbf{Q})$ be an element with $\mathbf{Q}$-irreducible characteristic polynomial whose eigenvalues are all real and distinct. Then $F:=\mathbf{Q}(\gamma) \subset M_{N}(\mathbf{Q})$ is a totally real number field of degree $[F: \mathbf{Q}]=N$ and $\mathbf{Q}^{N}$ is an $F$-vector space of $F$ dimension one. We fix a nonzero vector $v_{0} \in \mathbf{Q}^{N}$ and identify the vector spaces $F \simeq \mathbf{Q}^{N}$ via the map $\alpha \mapsto \alpha v_{0}$.

We write $\sigma_{1}, \ldots, \sigma_{N}: F \rightarrow \mathbf{R}$ for the distinct real embeddings of $F$. Then we have $V=\oplus_{1 \leq i \leq N} V_{i}$, where

$$
V_{i}:=V \otimes_{F, \sigma_{i}} \mathbf{R}=\operatorname{ker}\left(\gamma-\sigma_{i}(\gamma)\right)
$$


is the eigenspace associated with $\sigma_{i}(\gamma)$. Fix $g_{0} \in \mathrm{GL}_{N}(\mathbf{R})$ such that

$$
\gamma=g_{0}\left(\begin{array}{ccc}
\sigma_{1}(\gamma) & & \\
& \ddots & \\
& & \sigma_{N}(\gamma)
\end{array}\right) g_{0}^{-1} .
$$

Note that the decomposition (12.10) is orthogonal with respect to the quadratic form $\left(g_{0}^{\top}\right)^{-1} g_{0}^{-1}$ attached to $g_{0}$; it is more generally orthogonal with respect to the quadratic forms attached to any $g_{\gamma} g_{0}$ where $g_{\gamma}$ is an element of the centralizer $G_{\gamma}$ of $\gamma$ in $\mathrm{GL}_{N}(\mathbf{R})$.

We have

$$
G_{\gamma} \simeq(F \otimes \mathbf{R})^{\times}
$$

so that $G_{\gamma}$ is a maximal $\mathbf{R}$-split torus of $\mathrm{GL}_{N}(\mathbf{R})$. We orient $G_{\gamma}$ as follows. Our choice of real places of $F$ identifies $(F \otimes \mathbf{R})^{\times}$with $\left(\mathbf{R}^{\times}\right)^{N}$ and hence the tangent space $T_{x} G_{\gamma}$ for any $x \in G_{\gamma}$ with $\mathbf{R}^{N}$, and we require that the standard orientation on $\mathbf{R}^{N}$ corresponds to our orientation of $G_{\gamma}$. We assume that we have chosen the real places such that this is also the orientation induced by the standard orientation of $\mathbf{R}^{N}$ under the identification $F \simeq \mathbf{Q}^{N}$ given by $v_{0}$.

Let $G_{\gamma}^{\text {der }}=G_{\gamma}^{+} \cap \mathrm{SL}_{N}(\mathbf{R})$. The inclusion $G_{\gamma} \subset \mathrm{GL}_{N}(\mathbf{R})$ induces an injective map

$$
\begin{aligned}
X_{G_{\gamma}}:=G_{\gamma}^{\text {der }} \simeq G_{\gamma}^{+} / Z(\mathbf{R})^{+} \stackrel{\iota_{\gamma}}{\longrightarrow} \mathrm{GL}_{N}(\mathbf{R})^{+} / \mathrm{SO}(N) Z(\mathbf{R})^{+}=X \\
g_{\gamma} \mapsto g_{\gamma} g_{0} \mathrm{SO}(N) Z(\mathbf{R})^{+}
\end{aligned}
$$

The map $\iota_{\gamma}$ identifies $X_{G_{\gamma}}$ with the positive definite metrics in $X$ (up to rescaling) such that the decomposition $V=\oplus V_{i}$ above is orthogonal. For any congruence subgroup $\Gamma \subset \mathrm{SL}_{N}(\mathbf{Z})$, it induces a map

$$
\iota_{\gamma, \Gamma}: \Gamma \cap G_{\gamma}^{+} \backslash X_{G_{\gamma}} \rightarrow \Gamma \backslash X .
$$

The group $\Gamma \cap G_{\gamma}^{+}$has finite index in $\mathcal{O}_{F}^{\times}$, and so the quotient

$$
\Gamma \cap G_{\gamma}^{+} \backslash X_{G_{\gamma}} \cong \Gamma \cap G_{\gamma}^{+} \backslash(F \otimes \mathbf{R})_{+}^{1}
$$

is compact by Dirichlet's unit theorem. The pushforward under $\iota_{\gamma, \Gamma}$ of its fundamental class defines a homology class

$$
\left(\iota_{\gamma, \Gamma}\right)_{*}\left[\Gamma \cap G_{\gamma}^{+} \backslash X_{G_{\gamma}}\right] \in \mathrm{H}_{N-1}(\Gamma \backslash X, \mathbf{Z}) .
$$

Remark. In the case $N=2$ we have associated to any real quadratic field $\mathbf{Q}(\sqrt{d})$ a closed oriented geodesic. This depends on the choice of $\gamma$ or equivalently on the choice of a fractional ideal. If one restricts to primitive geodesics (or ideals) there are only finitely many conjugacy classes of such $\gamma$ 's. The cardinality of this finite set is the class number of $\mathbf{Q}(\sqrt{d})$.

12.5. Hecke characters of finite order. Let $\chi: G_{F} \rightarrow \overline{\mathbf{Q}}^{\times}$be a totally odd character of finite order. We use the same symbol $\chi$ to denote the corresponding Hecke character $\chi: F^{\times} \backslash \mathbf{A}_{F}^{\times} \rightarrow \overline{\mathbf{Q}}^{\times}$associated by class field theory. Here $\mathbf{A}_{F}$ denotes the adèles of $F$. We shall simply denote by $\mathbf{A}$ the adèles of $\mathbf{Q}$ and let $\omega$ be the restriction of $\chi$ to $\mathbf{A}^{\times}$.

When $N=2$ a Hecke character of $F=\mathbf{Q}(\sqrt{d})$ associates a rational number to each primitive geodesic. The totally odd hypothesis is natural: we want a geodesic and its opposite to be decorated with opposite numbers. In general, we similarly define a class

$$
\gamma_{\chi} \in \mathrm{H}_{N-1}\left(\left[\mathrm{GL}_{N}\right], \mathbf{Z}[\chi]\right) \text {. }
$$


Let $K_{\chi}=\operatorname{ker}\left(\left.\chi_{f}\right|_{\hat{\mathcal{O}}_{F}^{\times}}\right)$; thus $K_{\chi}$ is an open compact subgroup of $\hat{\mathcal{O}}_{F}^{\times}$. Let $F_{+}^{\times} \subset F^{\times}$ the subset of totally positive elements and write

$$
\mathbf{A}_{F, f}^{\times}=\coprod_{i} F_{+}^{\times} t_{i} K_{\chi}
$$

(finite union). Then

$$
F^{\times} \backslash \mathbf{A}_{F}^{1} / K_{\chi}=\coprod_{i} \Gamma_{i} \backslash(F \otimes \mathbf{R})_{+}^{1},
$$

where $\Lambda_{i}:=F_{+}^{\times} \cap t_{i} K_{\chi} t_{i}^{-1}$. The bijection sends $\Lambda_{i} t_{\infty}$ to $F^{\times}\left(t_{i}, t_{\infty}\right) K_{\chi}$. Define

$$
[\chi]=\sum_{i} \chi\left(t_{i}\right)\left[\Lambda_{i} \backslash(F \otimes \mathbf{R})_{+}^{1}\right] \in \mathrm{H}_{N-1}\left(F^{\times} \backslash \mathbf{A}_{F}^{1} / K_{\chi}, \mathbf{Z}[\chi]\right) .
$$

Consider the map $\mathbf{A}_{F}^{\times} \rightarrow \mathrm{GL}_{N}(\mathbf{A})$ induced by the inclusion $F^{\times}=\mathbf{Q}(\gamma)^{\times} \subset \mathrm{GL}_{N}(\mathbf{Q})$. If $K_{f} \subset \mathrm{GL}_{N}(\hat{\mathbf{Z}})$ is an open compact subgroup such that $K_{\chi} \subseteq K_{f} \cap \mathbf{A}_{F}^{\times}$, we obtain a well defined map

$$
\iota_{\gamma, K_{f}}: F^{\times} \backslash \mathbf{A}_{F}^{1} / K_{\chi} \simeq F^{\times} \backslash \mathbf{A}_{F}^{\times} / K_{\chi} \mathbf{R}_{>0} \rightarrow\left[\mathrm{GL}_{N}\right] / K_{f}
$$

and hence a class

$$
[\chi]_{K_{f}}:=\left(\iota_{\gamma, K_{f}}\right)_{*}[\chi] \in \mathrm{H}_{N-1}\left(\left[\mathrm{GL}_{N}\right] / K_{f}, \mathbf{Z}[\chi]\right) .
$$

Note that if $K_{f}^{\prime} \subseteq K_{f}$ is a finite index subgroup that still satisfies $K_{\chi} \subseteq K_{f}^{\prime} \cap \mathbf{A}_{F}^{\times}$, then the image of $[\chi]_{K_{f}^{\prime}}$ under the natural map $\left[\mathrm{GL}_{N}\right] / K_{f}^{\prime} \rightarrow\left[\mathrm{GL}_{N}\right] / K_{f}$ is $[\chi]_{K_{f}}$. Thus we have defined a class in the projective limit

$$
[\chi] \in \lim _{K_{f} \cap \mathbf{A}_{F}^{\times} \supseteq K_{\chi}} \mathrm{H}_{N-1}\left(\left[\mathrm{GL}_{N}\right] / K_{f}, \mathbf{Z}[\chi]\right) .
$$

\subsection{Period computations.}

Theorem 25. Let $\varphi_{f} \in \mathcal{S}_{0}\left(V\left(\mathbf{A}_{f}\right)\right)$ and assume that $\varphi_{f}$ is fixed by some open compact subgroup $K_{f}$ of $\mathrm{GL}_{N}(\hat{\mathbf{Z}})$ such that $K_{\chi} \subseteq K_{f} \cap \mathbf{A}_{F}^{\times}$. Then the global period

$$
\left[E_{\psi}\left(\omega, \varphi_{f}\right)\right]([\chi])=-L(\chi, 0)\left(\prod_{v \text { ramified }} Z_{v}\left(\varphi_{v}, \chi_{v}, 0\right)\right) .
$$

In the last product $v$ runs over all finite ramified places of $F$ and

$$
Z_{v}\left(\varphi_{v}, \chi_{v}, s\right):=\int_{F_{v}^{\times}} \chi_{v}\left(t_{v}\right)\left|t_{v}\right|_{v}^{s} \varphi_{v}\left(t_{v}^{-1} v_{0}\right) d^{\times} t_{v} .
$$

Since the Eisenstein class is a rational class if $\varphi_{f}$ and $\chi$ are rational, we get:

$$
\left[E_{\psi}\left(\omega, \varphi_{f}\right)\right]([\chi]) \in \mathbf{Q} .
$$

This implies classical rationality results for $L$-functions of characters.

Indeed, we may consider particular cases of these results where $\varphi_{f}$ is the characteristic function of a coset of a lattice and express this in more classical language: let $\mathfrak{f}$ and $\mathfrak{b}$ be two relatively prime ideals in the ring of integers $\mathcal{O}_{F}$. The partial zeta function attached to the ray class $\mathfrak{b}$ mod $\mathfrak{f}$ is defined by

$$
\zeta(\mathfrak{b}, \mathfrak{f}, s):=\sum_{\mathfrak{a} \equiv \mathfrak{b} \bmod \mathfrak{f}} \frac{1}{N(\mathfrak{a})^{s}}, \quad \operatorname{Re}(s)>1,
$$

where $\mathfrak{a}$ runs over all integral ideals in $\mathcal{O}_{F}$ such that the fractional ideal $\mathfrak{a} \mathfrak{b}^{-1}$ is a principal ideal generated by a totally positive number in the coset $1+\mathfrak{f b}^{-1}$. 
The fractional ideal $\mathfrak{f b}^{-1}$ defines a lattice $L$ in $F \otimes \mathbf{R}$. Applying the above corollary with $\varphi_{f}$ equal to the characteristic function of the coset $1+\mathfrak{f b}^{-1}$ we get:

Corollary 26 (Klingen, Siegel, Shintani).

$$
\zeta(\mathfrak{b}, \mathfrak{f}, 0) \in \mathbf{Q} .
$$

The Eisenstein class being almost integral our methods in fact imply the following integrality result.

Corollary 27 (Deligne-Ribet, Cassou-Noguès [22, 14]). Let $\mathfrak{c}$ be an integral ideal coprime to $\mathfrak{f b}^{-1}$. Then, we have:

$$
n(\mathfrak{c}) \zeta(\mathfrak{b}, \mathfrak{f}, 0)-\zeta(\mathfrak{b} \mathfrak{c}, \mathfrak{f}, 0) \in \mathbf{Z}\left[\frac{1}{n(\mathfrak{c})}\right] .
$$

Here $n$ denotes the norm.

The rest of this section is devoted to the proof of Theorem 25 .

12.7. Pullback of the transgression form. Consider the cartesian diagram

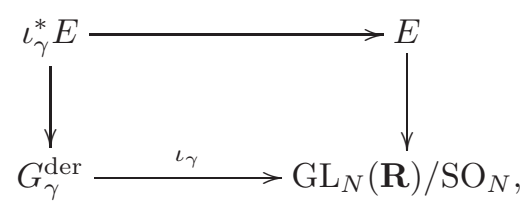

where $E$ is the universal $\mathrm{GL}_{N}(\mathbf{R})$-bundle $S \times V$ and $\iota_{\gamma}^{*} E$ carries the metric, orientation and equivariant structure induced by those of $E$. The pullback bundle $\iota_{\gamma}^{*} E$ also admits an action of $F \otimes \mathbf{R}$ compatible with its $G_{\gamma}^{\text {der }}$-equivariant structure, and this yields an equivariant splitting

$$
\iota_{\gamma}^{*} E \simeq \oplus_{1 \leq i \leq N} L_{i}
$$

where each $L_{i}$ is a euclidean line bundle and the sum is orthogonal. More concretely, the fiber of $L_{i}$ is the line $V_{i}$ in (12.10), and the metric $h_{L_{i}, \lambda}$ on the fiber of $L_{i}$ over $\lambda=$ $\left(\lambda_{1}, \ldots, \lambda_{N}\right) \in G_{\gamma}^{\text {der }} \subset \mathbf{R}_{>0}^{N}$ is given by

$$
h_{L_{i}, \lambda}=\lambda_{i}^{2} h_{L_{i}, 1} \text {. }
$$

We fix an orientation of each $L_{i}$ so that the natural isomorphism $\otimes L_{i} \simeq \wedge^{N} \iota_{\gamma}^{*} E$ induced by (12.24) preserves orientations. Using the functoriality of the Mathai-Quillen Thom form $\varphi=\varphi_{E} \in A^{N}(E)$, we find that

$$
\iota_{\gamma}^{*} \varphi=\iota_{\gamma}^{*} \varphi_{E}=\varphi_{\iota_{\gamma}^{*} E}=\varphi_{L_{1}} \wedge \cdots \wedge \varphi_{L_{N}} \in A^{N}\left(\iota_{\gamma}^{*} E\right) .
$$

For the transgression form $\psi=\psi_{E} \in A^{N-1}(E)$, we similarly find:

$$
\begin{aligned}
\iota_{\gamma}^{*} \psi_{E} & =\psi_{\iota_{\gamma}^{*} E} \\
& =\sum_{1 \leq i \leq n}(-1)^{i-1} \psi_{L_{i}} \varphi_{L_{1}} \wedge \cdots \wedge \widehat{\varphi_{L_{i}}} \wedge \cdots \wedge \varphi_{L_{n}} .
\end{aligned}
$$

As each $L_{i}$ is a trivial line bundle, from the formulas (6.1) and (7.5) we can write down explicit expressions for $\varphi_{L_{i}}$ and $\psi_{L_{i}}$. This is used in the next paragraph to compute the integral over $G_{\gamma}^{\text {der }}$. 
12.8. The integral over $G_{\gamma}^{\text {der }}$. Let $v \in \mathbf{R}^{N}$ and $v^{*} \psi_{E} \in A^{N-1}\left(\mathrm{GL}_{N}(\mathbf{R}) / \mathrm{SO}(N)\right)$ be the pullback of $\psi_{E}$ by the section of $E$ corresponding to $v$. We can now use the previous remarks to compute the integral

$$
Z_{\infty}\left(\psi_{E}, v, s\right):=\int_{G_{\gamma}^{\text {der }}} \int_{0}^{\infty} \iota_{\gamma}^{*}(t v)^{*} \psi_{E} t^{s} \frac{d t}{t}
$$

Using the diagram (12.23), we have

$$
Z_{\infty}\left(\psi_{E}, v, s\right)=\int_{G_{\gamma}^{\mathrm{der}}} \int_{0}^{\infty}(t v)^{*} \psi_{\iota_{\gamma}^{*} E} t^{s} \frac{d t}{t} .
$$

Write $v=\left(v_{1}, \ldots, v_{N}\right)$ according to the decomposition $\iota_{\gamma}^{*} E=\oplus L_{i}$ and consider $\lambda=$ $\left(\lambda_{1}, \ldots, \lambda_{N}\right) \in G_{\gamma}^{\text {der }}$. Using (12.25), (6.1) and (7.5) we compute

$$
\begin{aligned}
& v^{*} \varphi_{L_{i}}=(2 \pi)^{-1 / 2} e^{-\lambda_{i}^{2} v_{i}^{2} / 2} v_{i} d \lambda_{i} \\
& v^{*} \psi_{L_{i}}=-(2 \pi)^{-1 / 2} e^{-\lambda_{i}^{2} v_{i}^{2} / 2} v_{i} \lambda_{i}
\end{aligned}
$$

and so by (12.27)

$$
(t v)^{*} \psi_{\iota_{\gamma}^{*} E}=-(2 \pi)^{-N / 2} e^{-\sum_{i=1}^{N} \lambda_{i}^{2} t^{2} v_{i}^{2} / 2}\left(t v_{1}\right) \cdots\left(t v_{N}\right) \sum_{i=1}^{N}(-1)^{i-1} \lambda_{i} \wedge_{j \neq i} d \lambda_{j} .
$$

The change of variables $u_{i}=\lambda_{i} t$ gives $u_{1} \cdots u_{N}=t^{N}$ and $\frac{d u_{i}}{u_{i}}=\frac{d \lambda_{i}}{\lambda_{i}}+\frac{d t}{t}$, hence

$$
\frac{d t}{t} \wedge \sum_{i=1}^{N}(-1)^{i-1} \wedge_{j \neq i} \frac{d \lambda_{j}}{\lambda_{j}}=\frac{d u_{1} \wedge \cdots \wedge d u_{N}}{u_{1} \cdots u_{N}}
$$

and

$$
\begin{aligned}
Z_{\infty}\left(\psi_{E}, v, s\right) & =-(2 \pi)^{-N / 2} \int_{\mathbf{R}_{>0}^{N}} e^{-\sum_{i=1}^{N} u_{i}^{2} v_{i}^{2} / 2} v_{1} \cdots v_{N} \frac{d u_{1} \wedge \cdots \wedge d u_{N}}{\left(u_{1} \cdots u_{N}\right)^{-s / N}} \\
& =-\prod_{i=1}^{N} I\left(v_{i}, s\right)
\end{aligned}
$$

where

$$
\begin{aligned}
I(v, s) & =(2 \pi)^{-1 / 2} \int_{0}^{+\infty} e^{-v^{2} u^{2} / 2} v u^{s / N} d u \\
& \left.=\frac{1}{\pi^{\frac{1}{2}}} 2^{\frac{s}{2 N}-1} \Gamma\left(\frac{s}{2 N}+\frac{1}{2}\right)\right) \frac{\operatorname{sgn}(v)}{|v|^{s / N}} .
\end{aligned}
$$

Note that $I(v, 0)=\operatorname{sgn}(v) / 2$ and so $Z_{\infty}\left(\psi_{E}, v, 0\right)=-2^{-N} \prod_{i=1}^{N} \operatorname{sgn}\left(v_{i}\right)$.

12.9. Proof of Theorem 25: computation of the global period. Let $\varphi_{f} \in \mathcal{S}_{0}\left(V\left(\mathbf{A}_{f}\right)\right)$ and assume that $\varphi_{f}$ is fixed by some open compact subgroup $K_{f}$ of $\mathrm{GL}_{N}(\hat{\mathbf{Z}})$ such that $K_{\chi} \subseteq K_{f} \cap \mathbf{A}_{F}^{\times}$. Consider the integral

$$
Z\left(\varphi_{f}, \chi, s\right):=\int_{F \times \backslash \mathbf{A}_{F}^{1} / K_{\chi}} \chi(t) \cdot \iota_{\gamma, K_{f}}^{*} E\left(\omega, \varphi_{f}, s\right) .
$$

Its value at $s=0$ is

$$
Z\left(\varphi_{f}, \chi, 0\right)=\left[E\left(\omega, \varphi_{f}\right)\right]([\chi])
$$


Note that the integrand in $Z\left(\varphi_{f}, \chi, s\right)$ is defined on $\mathbf{A}_{F}^{\times}$and that it is invariant under $\hat{\mathbf{Z}}^{\times} \subset$ $\mathbf{A}^{\times}$, hence using that $K_{\chi} \cap \hat{\mathbf{Z}}^{\times}=K_{\omega}$, we have

$$
Z\left(\varphi_{f}, \chi, s\right)=\left[\hat{\mathbf{Z}}^{\times}: K_{\omega}\right] \int_{F \times \backslash \mathbf{A}_{F}^{1} / K_{\chi} \hat{\mathbf{Z}} \times} \chi(t) \cdot \iota_{\gamma, K_{f}}^{*} E\left(\omega, \varphi_{f}, s\right) .
$$

Since $\mathbf{A}_{F}^{\times}=\mathbf{A}_{F}^{1} \mathbf{A}^{\times}$and $F^{\times} K_{\chi} \hat{\mathbf{Z}}^{\times}=\mathbf{A}_{F}^{1} \cap F^{\times} \mathbf{A}^{\times} K_{\chi}$, we can write

$$
F^{\times} \backslash \mathbf{A}_{F}^{1} / K_{\chi} \hat{\mathbf{Z}}^{\times} \simeq F^{\times} \mathbf{A}^{\times} \backslash \mathbf{A}_{F}^{\times} / K_{\chi} .
$$

Assume that $\varphi_{f}=\otimes_{w} \varphi_{w}$ where $w$ runs over the non-archimedean places of $F$. Then we have

$$
\begin{aligned}
{\left[\hat{\mathbf{Z}}^{\times}: K_{\omega}\right]^{-1} Z\left(\varphi_{f}, \chi, s\right) } & =\int_{\mathbf{A}^{\times} F^{\times} \backslash \mathbf{A}_{F}^{\times} / K_{\chi}} \chi(t) \cdot E\left(t, \omega, \varphi_{f}, s\right) \quad(\text { by }(12.37) \text { and (12.38)) } \\
& =\int_{\mathbf{A}^{\times} F^{\times} \backslash \mathbf{A}_{F}^{\times} / K_{\chi}} \chi(t) \int_{\mathbf{Q}^{\times} \backslash \mathbf{A}^{\times} / K_{\omega}} \theta^{*}\left(z t ; \varphi_{f}\right) \omega(z)|z|^{N s} \frac{d z_{\infty}}{z_{\infty}} \\
& =\int_{\mathbf{A}^{\times} F^{\times} \backslash \mathbf{A}_{F}^{\times} / K_{\chi}} \int_{\mathbf{Q}^{\times} \backslash \mathbf{A}^{\times} / K_{\omega}} \chi(z t)|n(z t)|^{s} \theta^{*}\left(z t ; \varphi_{f}\right) \frac{d z_{\infty}}{z_{\infty}} \\
& =\int_{F^{\times} \backslash \mathbf{A}_{F}^{\times} / K_{\chi}} \chi(t)|n(t)|^{s} \theta^{*}\left(t ; \varphi_{f}\right) \frac{1}{N} \frac{d n\left(t_{\infty}\right)}{n\left(t_{\infty}\right)} \\
& \left(\operatorname{since} \mathbf{Q}^{\times} K_{\omega} \backslash \mathbf{A}^{\times} \simeq F^{\times} K_{\chi} \backslash \mathbf{A}^{\times} F^{\times} K_{\chi}\right) \\
& =\int_{F^{\times} \backslash \mathbf{A}_{F}^{\times} / K_{\chi}} \chi(t)|n(t)|^{s}\left(\sum_{x \in F^{\times}} \varphi_{f}\left(t_{f}^{-1} x v_{0}\right) \psi\left(t_{\infty}^{-1} x v_{0}\right)\right) \frac{1}{N} \frac{d n\left(t_{\infty}\right)}{n\left(t_{\infty}\right)} \\
& =\int_{\mathbf{A}_{F}^{\times} / K_{\chi}} \chi(t)|n(t)|^{s} \varphi_{f}\left(t_{f}^{-1} v_{0}\right) \psi\left(t_{\infty}^{-1} v_{0}\right) \frac{1}{N} \frac{d n\left(t_{\infty}\right)}{n\left(t_{\infty}\right)} \\
& =\prod_{w \nmid \infty} Z_{w}\left(\varphi_{w}, \chi_{w}, s\right) \cdot Z_{\infty}\left(\psi, \chi_{\infty}, s\right)
\end{aligned}
$$

where

$$
\begin{aligned}
& Z_{w}\left(\varphi_{w}, \chi_{w}, s\right)=\int_{F_{w}^{\times}} \chi_{w}\left(t_{w}\right)\left|t_{w}\right|_{v}^{s} \varphi_{w}\left(t_{w}^{-1} v_{0}\right) d^{\times} t_{w}, \\
& Z_{\infty}\left(\psi, \chi_{\infty}, s\right)=\int_{F_{\infty}^{\times}} \chi_{\infty}\left(t_{\infty}\right)\left|t_{\infty}\right|_{\infty}^{s} \psi\left(t_{\infty}^{-1} v_{0}\right) \frac{1}{N} \frac{d n\left(t_{\infty}\right)}{n\left(t_{\infty}\right)},
\end{aligned}
$$

and the measure $d^{\times} t_{w}$ for non-archimedean $w$ is normalized so that the volume of $K_{\chi, w}$ is one. For unramified $w$, writing $\pi_{w}$ for a uniformizer of $\mathcal{O}_{F_{w}}$ and $q_{w}=\left|\mathcal{O}_{F_{w}} / \pi_{w} \mathcal{O}_{F_{w}}\right|$, we have

$$
Z_{w}\left(\varphi_{w}, \chi_{w}, s\right)=L\left(\chi_{w}, s\right)=\left(1-\chi_{w}\left(\pi_{w}\right) q_{w}^{-s}\right)^{-1} .
$$

For the archimedean integral, since $F_{\infty}^{\times}$has $2^{N}$ connected components and the integrand transforms like sgn under the action of $\{ \pm 1\}^{N}$, using (12.33) we obtain

$$
Z_{\infty}\left(\psi, \chi_{\infty}, 0\right)=-1
$$

(recall that with our choices of orientations, writing $v_{0}=\left(v_{1}, \ldots, v_{N}\right)$ we have $\operatorname{sgn}\left(v_{1}\right) \cdots \operatorname{sgn}\left(v_{N}\right)=$ 1). This concludes the proof of Theorem 25. 


\section{Eisenstein theta CORRESPONDENCE FOR THE DUAL PAIR $\left(\mathrm{GL}_{a}, \mathrm{GL}_{b}\right)$}

We can think of our construction of the closed differential form $E_{\psi}$ as a regularized theta lift

$$
E_{\psi}\left(\omega, \varphi_{f}\right)=\left(\omega, \theta\left(\varphi_{f} \otimes \psi\right)\right)^{\mathrm{reg}} \in A^{N-1}\left(\left[\mathrm{GL}_{N}\right]\right)
$$

of the character $\omega$, for the dual pair $\left(\mathrm{GL}_{N}, \mathrm{GL}_{1}\right)$ of type II. This suggests looking more generally at dual pairs $\left(\mathrm{GL}_{a}, \mathrm{GL}_{b}\right)$. In this last section we discuss some examples, focusing on the case $a=N, b=2$.

13.1. Dual pairs. For a local field $F$, the group $\mathrm{GL}_{N}(F) \times \mathrm{GL}_{2}(F)$ acts on $F^{N} \otimes_{F} F^{2}$. If we rewrite this tensor product as $M_{N, 2}(F)(N \times 2$ matrices), the action is given by

$$
(A, B) \cdot x=A x B^{-1} \quad\left((A, B) \in \mathrm{GL}_{N}(F) \times \mathrm{GL}_{2}(F), x \in M_{N, 2}\right) .
$$

Denote by $\omega$ the induced action on the Schwartz space $\mathcal{S}\left(F^{N} \otimes_{F} F^{2}\right)$, given by

$$
\omega(A, B) \varphi(x)=\varphi\left(A^{-1} x B\right) .
$$

The canonical identification of $F^{N} \otimes_{F} F^{2}$ with the $2 N$-dimensional vector space $F^{2 N}$ yields an embedding of $\mathrm{GL}_{N}(F) \times \mathrm{GL}_{2}(F)$ into $\mathrm{GL}_{2 N}(F)$. In this way $\omega$ is just the restriction of the Weil representation considered above, and we can use $\theta\left(\varphi_{f} \otimes \psi\right)$ as a kernel for a regularized theta lift. We describe an explicit incarnation of this lift in the next paragraph. Before that, we first describe in more detail (and in classical terms) the dual pair above when $F=\mathbf{Q}$.

Let us start by saying that the reader may have been surprised by the non standard coordinates $(\tau, z)$ of $\$ 6.2$. We may now explain our choice: we have worked the whole time with the dual pair $\left(\mathrm{GL}_{N}, \mathrm{GL}_{1}\right)$, we could however have worked with $\left(\mathrm{GL}_{1}, \mathrm{GL}_{N}\right)$. In the latter case $\mathrm{GL}_{N}(\mathbf{R})$ acts on the left on $\mathbf{R}^{N}=M_{1, N}(\mathbf{R})$, and when $N=2$ this corresponds to the standard coordinates $(\tau, u \tau+v)$ on $\mathcal{H} \times \mathbf{C}$.

Let us now discuss the dual pair $\left(\mathrm{GL}_{N}, \mathrm{GL}_{2}\right)$ so that $\mathrm{GL}_{2}$ acts from the right. Let $V=$ $\mathbf{Q}^{N}$ and fix a lattice $L$ in $V$. With respect to the (now standard) coordinates $(\tau, z=u \tau+v)$ on $\mathcal{H} \times \mathbf{C}$, an element

acts by

$$
B=\left(\begin{array}{ll}
a & b \\
c & d
\end{array}\right) \in \mathrm{GL}_{2}(\mathbf{R})^{+}
$$

$$
B:(\tau, z=u \tau+v) \mapsto\left(\frac{a \tau+b}{c \tau+d}, \frac{z}{c \tau+d}\right) .
$$

It induces a diagonal action of $\mathrm{GL}_{2}(\mathbf{R})^{+}$on

$$
\mathcal{H} \times(V \otimes \mathbf{C})=\mathcal{H} \times \mathbf{C}^{N}
$$

which commutes with the left linear action of $\mathrm{GL}_{N}(\mathbf{R})$ on $\mathbf{C}^{N}$.

The $\mathbf{Z}^{2}$-action on $\mathcal{H} \times \mathbf{C}$

$$
(u, v) \cdot(\tau, z)=(\tau, z+u \tau+v)
$$

induces an action of $L \otimes \mathbf{Z}^{2}$ on

$$
\mathcal{H} \times \mathbf{C}^{N}=\mathcal{H} \times(L \otimes \mathbf{C}) .
$$

Any congruence subgroup of the modular group $\mathrm{SL}_{2}(\mathbf{Z})=\mathrm{GL}_{2}(\mathbf{Z})^{+}$acts on the quotient

$$
\left(\mathcal{H} \times \mathbf{C}^{N}\right) /\left(L \otimes \mathbf{Z}^{2}\right) .
$$

The quotient space is the total space of the $N$-fold fiber product $\mathcal{E}^{N}$ of the universal elliptic curve $\mathcal{E}$ over the corresponding modular curve $Y$. 
Let $\Gamma$ be a subgroup of $\mathrm{GL}(V)^{+}$that fixes $L$. The group $\Gamma$ acts linearly (on the left) on (13.3) and its action commutes with the (right) action of the modular group. This yields a natural action of $\Gamma$ on $\mathcal{E}^{N}$, and a diagonal action on $X \times \mathcal{E}^{N}$. The quotient space

$$
\Gamma \backslash\left(X \times \mathcal{E}^{N}\right)
$$

embeds naturally into the universal family $\mathcal{T}$ of metrized $2 N$-dimensional tori associated to $V \otimes \mathbf{C}$ and $L \otimes \mathbf{Z}^{2}$. We will now consider the restriction of the form $E_{\psi}$ constructed in Section 8 to (13.4) minus the zero section.

13.2. A regularized theta lift. Locally the quotient (13.4) splits as a product that remembers both the splitting

$$
X \times \mathcal{H} \times \mathbf{C}^{N}
$$

and the complex structure on the last factor. Though this splitting doesn't descend to (13.4), it induces a splitting of tangent spaces and therefore also a splitting at the level of differential forms. Write

$$
E_{\psi}=\sum_{a+b+c+d=2 N-1} E_{\psi}^{(a, b, c, d)}
$$

for the corresponding decomposition, so that $E_{\psi}^{(a, b, c, d)}$ is of degree $a$ along $X$, degree $b$ along $\mathcal{H}$ and bi-degree $(c, d)$ along $\mathbf{C}^{N}$.

Denote by $d z_{i}=\tau d u_{i}+d v_{i}(i=1, \ldots, N)$. The $N$-form $d z_{1} \wedge \ldots \wedge d z_{N}$ on the space (13.5) is of type $(0,0, N, 0)$. It is both $\left(L \otimes \mathbf{Z}^{2}\right)$ - and $\Gamma$-invariant, and is multiplied by $(c \tau+d)^{-N}$ under the action of an element $\left(\begin{array}{ll}a & b \\ c & d\end{array}\right) \in \mathrm{GL}_{2}(\mathbf{R})^{+}$. It follows that the quotient

$$
E_{\psi}^{(N-1,0, N, 0)} / d z_{1} \wedge \ldots \wedge d z_{N}
$$

defines a $\Gamma$-invariant differential form in

$$
A^{N-1}\left(X, A^{0}\left(\mathcal{E}^{N}-\{0\}, \mathcal{L}^{\otimes N}\right)\right) \text {. }
$$

Here $\mathcal{L}$ is the line bundle over $\mathcal{E}^{N}$ obtained as the pull back by the projection $\mathcal{E}^{N} \rightarrow Y$ of the Hodge line bundle over $Y$, i.e. the pullback of $\mathcal{O}(-1)$ under the natural inclusion $\mathcal{H} \subset \mathbf{P}^{1}(\mathbf{C})$, and the action of $\Gamma$ on the space $A^{0}\left(\mathcal{E}^{N}, \mathcal{L}^{\otimes N}\right)$ of sections of $\mathcal{L}^{\otimes N}$ is induced by the, respectively linear and trivial, actions of $\Gamma$ on $\mathcal{E}^{N}$ and $Y$.

Evaluated on a section $x: Y \rightarrow \mathcal{E}^{N}$, an element of $A^{0}\left(\mathcal{E}^{N}, \mathcal{L}^{\otimes N}\right)$ gives a section of the Hodge line bundle over $Y$. It follows that any nonzero torsion section of $x: Y \rightarrow \mathcal{E}^{N}$ gives rise to a form $E_{\psi, x}^{(N-1,0, N, 0)}$ of degree $N-1$ that takes values in the space of sections of the Hodge line bundle over $Y$. After some computations in $(\mathfrak{g}, K)$-cohomology, one can prove the following:

Lemma 28. The $(N-1)$-form

$$
E_{\psi, x}^{(N-1,0, N, 0)} \in A^{N-1}\left(X, A^{0}\left(Y, \mathcal{L}^{\otimes N}\right)\right)
$$

is closed (along $X$ ).

Remark. In case $N=1$, the dual pair is $\left(\mathrm{GL}_{1}, \mathrm{GL}_{2}\right)$, the statement about closedness is empty since then $X$ is just a point. However, the component $E_{\psi}^{(N-1,0, N, 0)}$ is already interesting: it corresponds to the form $E_{1}(\tau, z) d z$.

The case of the dual pair $\left(\mathrm{GL}_{2}, \mathrm{GL}_{1}\right)$ does not fall into a case above. The total space is a quotient of $\mathcal{H} \times\{\mathrm{pt}\} \times \mathbf{R}^{2}$ where we don't emphasize the complex structure on the last factor. The induced splitting then only involves three parameters $(a, b, c)$ with $a+b+c=1$. 
However there is a corresponding closedness lemma that we have already observed: for any $(u, v) \in \mathbf{R}^{2}$,

$$
E_{\psi,(u, v)}^{(1,0,0)}=E_{2}(\tau, u-\tau v) d \tau
$$

indeed defines a closed 1 -form on $\mathcal{H}$.

It follows from the discussion above that any $\Gamma$-invariant nonzero torsion section $x$ : $Y \rightarrow \mathcal{E}^{N}$ gives rise to a closed $(N-1)$-form $E_{\psi, x}^{(N-1,0, N, 0)}$ on $\Gamma \backslash X$ that takes values in the space of sections of the Hodge line bundle over $Y$. Using properties of Howe's theta correspondence, one can finally prove:

Theorem 29. Integration of $E_{\psi, x}^{(N-1,0, N, 0)}$ gives a non-trivial lift

$$
\Lambda_{\psi, x}: H_{N-1}(\Gamma \backslash X) \rightarrow M_{N}(Y) .
$$

Here $M_{N}(Y)$ denotes the space of weight $N$ modular forms on $Y$.

The lift $\Lambda_{\psi, x}$ is a de Rham version of the map (1.6) of the Introduction. It is a particular case of a 'regularized $\theta$-lift.' The general theory of the theta correspondence is a powerful tool to study these lifts. In the next paragraph we explain how to use it to evaluate our lift on certain homology classes. This shows in particular that $\Lambda_{\psi, x}$ is non-trivial in general.

13.3. Evaluation on tori. Let $F / \mathbf{Q}$ be a totally real number field of degree $N$. Denote by $\mathcal{O}_{F}$ its ring of integers. It corresponds to $F$ a global dual pair

$$
\left(F^{\times}, \mathrm{GL}_{2}(F)\right)
$$

in $\mathrm{GL}_{2 N}(\mathbf{Q})$. In the terminology of Kudla [37] we even have a seesaw of dual pairs in $\mathrm{GL}_{2 N}(\mathbf{Q})$ :

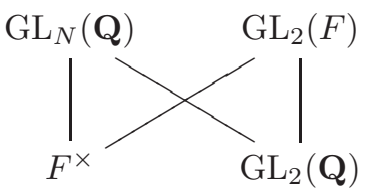

The corresponding seesaw identity allows us to evaluate the map

$$
H_{N-1}(\Gamma \backslash X, \mathbf{R}) \rightarrow M_{N}(Y)
$$

of the preceding paragraph on the homology class associated to $F^{\times}$. By Dirichlet units theorem the latter is an $(N-1)$-dimensional torus since $F$ is a totally real number field of degree $N$.

Let us first define the lattices associated to the pair $\left(\mathrm{GL}_{2}(F), F^{\times}\right)$in the seesaw (13.9).

Write $F=\mathbf{Q}(\gamma)$ as in $\S 12.4$. In the dual pair $\left(F^{\times}, \mathrm{GL}_{2}(F)\right)$ we associate to $F^{\times} \subset$ $\mathrm{GL}_{N}(\mathbf{Q})$ a quotient

$$
\Gamma \cap G_{\gamma}^{+} \backslash X_{\gamma} \subset \Gamma \backslash X .
$$

Let us now consider the $\mathrm{GL}_{2}(F)$-side: define the group

$$
\mathrm{GL}_{2}^{+}\left(\mathcal{O}_{F}\right)=\left\{\left(\begin{array}{ll}
a & b \\
c & d
\end{array}\right) \in \mathrm{GL}_{2}(F): a, b, c, d \in \mathcal{O}_{F}, a d-b c \in \mathcal{O}_{F}^{+, \times}\right\} .
$$

We identify the center of $\mathrm{GL}_{2}^{+}\left(\mathcal{O}_{F}\right)$ with $\mathcal{O}_{F}^{\times}$and let $\operatorname{PGL}_{2}^{+}\left(\mathcal{O}_{F}\right)$ be the quotient. The group $\mathrm{GL}_{2}^{+}\left(\mathcal{O}_{F}\right)$ acts on $(F \otimes \mathbf{R})^{2} \cong F \otimes \mathbf{C}$ from the right and stabilizes the lattice $\mathcal{O}_{F}^{2}$.

Identifying $F$ with $\mathbf{Q}^{N}$ as in $\S 12.4$ we may, and will from now on, suppose that $L=\mathcal{O}_{F}$ so that

$$
L \otimes \mathbf{Z}^{2}=\mathcal{O}_{F}^{2}
$$


We consider the $2 N$-torus bundle

$$
\mathcal{H}^{N} \times\left(F \otimes \mathbf{R} / \mathcal{O}_{F}\right)^{2}
$$

over

$$
\begin{aligned}
\mathcal{H}^{N} & =\mathcal{H} \times \cdots \times \mathcal{H} \\
& =F \otimes \mathcal{H}=\{\tau \in F \otimes \mathbf{C}: \operatorname{Im}(\tau) \text { totally positive }\}
\end{aligned}
$$

Note that the group $\mathrm{GL}_{2}^{+}(F \otimes \mathbf{R})$ acts simply transitively from the right on

$$
\left\{\left(\omega_{1}, \omega_{2}\right) \in(F \otimes \mathbf{C})^{2}: \operatorname{Im}\left(\omega_{1} / \omega_{2}\right)>0\right\}
$$

so that the map $\left(\omega_{1}, \omega_{2}\right) \mapsto \tau=\omega_{1} / \omega_{2}$ identifies the quotient

$$
\mathrm{GL}_{2}^{+}(F \otimes \mathbf{R}) /(F \otimes \mathbf{C})^{\times}=\mathrm{GL}_{2}^{+}(\mathbf{R}) / \mathrm{SO}_{2} Z_{2}^{+} \times \cdots \times \mathrm{GL}_{2}^{+}(\mathbf{R}) / \mathrm{SO}_{2} Z_{2}^{+}
$$

with $\mathcal{H}^{N}$.

Suppose now that the torsion section $x: Y \rightarrow \mathcal{E}^{N}$ of the preceding paragraph is associated to a nonzero torsion point in $(F \otimes \mathbf{R})^{2} / \mathcal{O}_{F}^{2}$ and let $\Lambda \subset \mathrm{GL}_{2}^{+}\left(\mathcal{O}_{F}\right)$ be the stabilizer of this torsion point. Let $\Delta=\Lambda \cap \mathcal{O}_{F}^{\times}$be the intersection of $\Lambda$ with the center. Then $\Lambda \subset \operatorname{GL}_{2}^{+}\left(\mathcal{O}_{F}\right)$ and $\Delta \subset \mathcal{O}_{F}^{\times}$are subgroups of finite index and we define $\Lambda^{\prime}=\Lambda / \Delta \subset \mathrm{PGL}_{2}^{+}\left(\mathcal{O}_{F}\right)$. Note that $\Delta$ is contained in $\Gamma \cap G_{\gamma}^{+}$.

Let $\mathcal{A}$ be the universal Abelian variety over the Hilbert modular curve of level $\Lambda^{\prime}$, in other words

$$
\mathcal{A}=\Lambda^{\prime} \backslash\left[\mathcal{H}^{N} \times\left(F \otimes \mathbf{R} / \mathcal{O}_{F}\right)^{2}\right] .
$$

The group $\Delta$ acts on $\mathcal{A}$, and the global space corresponding to the dual pair $\left(F^{\times}, \mathrm{GL}_{2}(F)\right)$ is now

$$
\Delta \backslash\left(X_{G_{\gamma}} \times \mathcal{A}\right)=\Delta \times \Lambda^{\prime} \backslash\left[X_{G_{\gamma}} \times \mathcal{H}^{N} \times\left(F \otimes \mathbf{R} / \mathcal{O}_{F}\right)^{2}\right] .
$$

The analogue of the splitting (13.5) for this dual pair is the product

$$
X_{G_{\gamma}} \times(\mathcal{H} \times \mathbf{C})^{N} .
$$

We then have an analogue of Theorem 29: the torsion section $x$ defines a nonzero section

$$
\Lambda^{\prime} \backslash \mathcal{H}^{N} \rightarrow \mathcal{A} .
$$

Consider the restriction of $E_{\psi}$ to $\mathcal{A}-\{0\}$, and pull back by $x$ its component of degree $N-1$ along $X_{G_{\gamma}}$. It defines a closed form on $\Delta \backslash X_{G_{\gamma}}$ that yields a lift which maps the fundamental class in $H_{N-1}\left(\Delta \backslash X_{G_{\gamma}}\right)$ to a Hecke-Eisenstein modular form of weight $(1, \ldots, 1)$ and of level $\Lambda^{\prime}$.

Remark. Pulling back the total form $E_{\psi}$ by a global section of $\mathcal{T}$ yields a different lift from $H_{N-1}\left(\Delta \backslash X_{G_{\gamma}}\right)$ to the Eisenstein cohomology of Hilbert modular varieties. This lift is also interesting; it is considered in great generality by Graf in his thesis, see [28] and [2, $\S 5.5]$. This leads to an integral version of all of Harder's Eisenstein cohomology. 
Proceeding as in $\S 12.8$, the explicit computation of the Hecke-Eisenstein modular form lifted from of the fundamental class in $H_{N-1}\left(\Delta \backslash X_{G_{\gamma}}\right)$ reduces to the following integral:

$$
\begin{aligned}
& \int_{\Delta \backslash(F \times \otimes \mathbf{Q} \mathbf{R})^{+}} \int_{0}^{\infty} t^{2 N s} \theta_{\psi_{1} \wedge \ldots \wedge \psi_{N}}(\mu, \tau, x)\left(u_{1} \cdots u_{N}\right)^{2 N s} d u_{1} \wedge \ldots \wedge d u_{N} \\
= & \sum_{(m, n) \in \mathcal{O}_{F}^{2}} \int_{\Delta \backslash\left(\mathbf{R}_{>0}\right)^{N}} \bigwedge_{j=1}^{N} \psi_{j}\left(u_{j}\left(x_{j}+m_{j} \tau_{j}+n_{j}\right)\right)\left(u_{1} \cdots u_{N}\right)^{2 N s} d u_{1} \wedge \ldots \wedge d u_{N} \\
= & \sum_{(m, n) \in \mathcal{O}_{F}^{2} / \Delta} \int_{\left(\mathbf{R}_{>0}\right)^{N}} \bigwedge_{j=1}^{N} \psi_{j}\left(u_{j}\left(x_{j}+m_{j} \tau_{j}+n_{j}\right)\right)\left(u_{1} \cdots u_{N}\right)^{2 N s} d u_{1} \wedge \ldots \wedge d u_{N} .
\end{aligned}
$$

Here $\psi_{j}$ is our form $\psi$ on the $j$-th factor $\mathcal{H}$ of $\mathcal{H}^{N}$, and we have denoted by $m_{j}$ and $n_{j}$ the images of $m$ and $n$ in $\mathbf{R}$ given by the $j$-th embedding of $F$ in $\mathbf{R}$. Finally, the last equality follows from the fact that $\Delta$ fixes the torsion point $x$.

Using the explicit form of $\psi_{j}$ given by (7.6) we conclude that the weight $N$ component of the result of this period calculation, is

$$
\left(2^{s-1} \Gamma(s+1) \frac{i}{\pi}\right)^{N}\left(\prod_{j=1}^{N} \operatorname{Im}\left(\tau_{j}\right)^{s}\right) \sum_{(m, n) \in \mathcal{O}_{F}^{2} / \Delta} \prod_{j=1}^{N} \frac{\overline{x_{j}+m_{j} \tau_{j}+n_{j}}}{\left|x_{j}+m_{j} \tau_{j}+n_{j}\right|^{2 s+2}} .
$$

Taking $s=0$ one indeed gets a Hecke-Eisenstein modular form of weight $(1, \ldots, 1)$ for the congruence subgroup $\Lambda^{\prime}$ of the Hilbert modular group $\mathrm{PGL}_{2}^{+}\left(\mathcal{O}_{F}\right)$.

Now, the geometric content of the seesaw (13.9) is that the diagram

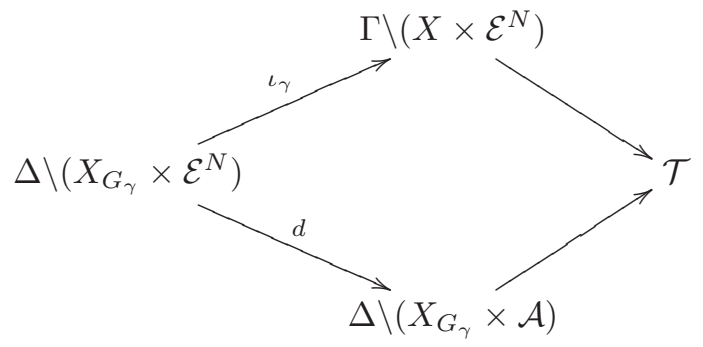

is commutative. Here $\iota_{\gamma}$ is induced by the inclusion $X_{G_{\gamma}} \subset X$ and $d$ is induced by the diagonal embedding $\mathcal{H} \subset \mathcal{H}^{N}$. Identifying the two different ways to restrict $E_{\psi}$ to

$$
\Delta \backslash\left(X_{G_{\gamma}} \times \mathcal{E}^{N}-\{0\}\right)
$$

we finally get Theorem 6 of the Introduction:

Theorem 30. The evaluation of $\Lambda_{\psi, x}$ on the image in $H_{N-1}(\Gamma \backslash X, \mathbf{Z})$ of the fundamental class in $H_{N-1}\left(\Delta \backslash X_{G_{\gamma}}, \mathbf{Z}\right)$ is a modular form of weight $N$ obtained as the restriction to the diagonal $\mathcal{H} \subset \mathcal{H}^{N}$ of a Hecke-Eisenstein modular form of weight $(1, \ldots, 1)$ for a congruence subgroup of $\mathrm{PGL}_{2}\left(\mathcal{O}_{F}\right)$.

13.4. Beyond totally real number fields. Cocycle methods have yet essentially been limited to totally real number fields. Let us now explain why we believe our tools should still be useful in general.

Let $K$ be an imaginary quadratic field. One may similarly associate to the dual pair $\left(\mathrm{GL}_{N}(K), K^{\times}\right)$in $\mathrm{GL}_{2 N}(\mathbf{Q})$ a lift from the degree $N-1$ homology groups of some 
congruence subgroups of $\mathrm{GL}_{N}(K)$ to Hecke characters of $K$ of type $\bar{z} / z$ at infinity. This leads to complex valued $(N-1)$-cocycles on congruence subgroups of $\mathrm{GL}_{N}\left(\mathcal{O}_{K}\right){ }^{6}$

A computation similar to that of $\S 12.8$ furthermore implies that the evaluation of such a cocycle on homology classes associated to a degree $N$ extension $F / K$, and a Dirichlet character $\chi$ of $F$ is, up to a nonzero constant in $\overline{\mathbf{Q}}$, equal to some special value of a (partial) $L$-function.

Example in the $N=2$ case. Let $L$ be a lattice in $\mathbf{C}$ with complex multiplication by $\mathcal{O}_{K}$.

Using the expression (7.8) for $\psi$, one can get an explicit expression for the cohomological Eisenstein series that realizes the lift we just alluded to: given two torsion points $u, v \in \mathbf{C} / L$, we obtain a complex differentiable 1 -form

$$
E_{\psi,(u, v)}(q, s)=E_{z}(q, s ; u, v) d z+E_{y}(q, s ; u, v) d t+E_{\bar{z}}(q, s ; u, v) d \bar{z}
$$

where ${ }^{7}$

$$
\begin{aligned}
E_{z}(q, s ; u, v) & =\Gamma(2 s+2) y^{2 s} \sum_{m \in L+u, n \in L+v} \frac{(\overline{n-z m})^{2}}{\left(|n-z m|^{2}+|m y|^{2}\right)^{2 s+2}}, \\
E_{\bar{z}}(q, s ; u, v) & =-\Gamma(2 s+2) y^{2 s} \sum_{m \in L+u, n \in L+v} \frac{(\bar{m} y)^{2}}{\left(|n-z m|^{2}+|m y|^{2}\right)^{2 s+2}},
\end{aligned}
$$

and

$$
E_{y}(q, s ; u, v)=-2 \Gamma(2 s+2) y^{2 s} \sum_{m \in L+u, n \in L+v} \frac{(\overline{n-z m}) \bar{m} y}{\left(|n-z m|^{2}+|m y|^{2}\right)^{2 s+2}} .
$$

That form can be meromorphically continued in the variable $s$; it is well defined at $s=0$ and becomes a closed 1-form $\omega(u, v)$. These forms were first considered by Hiroshi Ito [34] who proved that the integral of $\omega(u, v)$ along the cycle $Z_{A}$ associated to a hyperbolic matrix $A=\left(\begin{array}{ll}a & b \\ c & d\end{array}\right) \in \mathrm{SL}_{2}\left(\mathcal{O}_{K}\right)\left((a+d)^{2} \neq 0,1,4\right)$ that preserves $L$ and fixes $(u, v)$ (mod. $\left.L^{2}\right)$ is given by

$$
\int_{Z_{A}} \omega(u, v)= \pm\left(\alpha-\alpha^{\prime}\right) L(A, 1 ; u, v) .
$$

Here $\alpha$ and $\alpha^{\prime}$ are the two distinct solutions of the equation $c X^{2}+(d-a) X-b=0$ in C, the quadratic form

$$
Q(m, n)=(m \alpha+n)\left(m \alpha^{\prime}+n\right)
$$

and

$$
L(A, s ; u, v)=\sum_{(m, n)} \frac{\overline{Q(m, n)}}{|Q(m, n)|^{2 s}},
$$

where $(m, n)$ runs through a complete set of elements of $(L+u) \times(L+v)-\{(0,0)\}$ not associated with respect to the action of $A$. This $L$-series is absolutely convergent for $\operatorname{Re}(s)>3 / 2$ and has an analytic continuation to the whole $s$-plane.

Our viewpoint is well suited to study integrality features of the cohomology classes associated to the above complex cocycles. This leads to a new proof of the general conjecture

\footnotetext{
${ }^{6}$ A recent work by Florez, Karabulut and Wong [24], generalising Sczech's Eisenstein cocycle to extensions of an imaginary quadratic fields, provides a different construction of a similar degree $N-1$ cocycle.

${ }^{7}$ Compare to (7.8) there is a missing factor $-(4 \pi)^{2}=(2 \pi i)^{2}$; this normalisation is made to have integral periods and is reminiscent of the factor $2 \pi i$ factor in equation (11.6).
} 
of Deligne [21] for characters, ${ }^{8}$ and establishes that the corresponding $L$-values are 'almost integral' as in Corollary 27.

Consider finally the seesaw

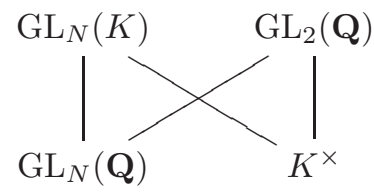

obtained by seeing $K^{N}$ either as a $K$-module or as a Q-module. It relates the lifts of $\S 13.2$ to the complex cocycles on congruence subgroups of $\mathrm{GL}_{N}(K)$ : the latters map $N$-tuples of matrices in $\mathrm{GL}_{N}(\mathbf{Q})$ to values of weight $N$ modular forms at CM points.

Example. Before Ito's work Sczech [42] had introduced a related complex-valued 1cocycle; we will refer to it as the Sczech elliptic cocycle. Ito indeed proves that this other - a priori unrelated - Sczech cocycle is associated to the differential 1-form $\omega(u, v)$ arising from (13.12). The seesaw identity associated to (13.14) implies that Sczech elliptic cocycle evaluated on a hyperbolic matrix in $\mathrm{SL}_{2}(\mathbf{Z})$ is equal to the evaluation at a $\mathrm{CM}$ point of a weight 2 modular form on $\mathcal{H}$ obtained as the restriction to the diagonal $\mathcal{H} \subset \mathcal{H}^{2}$ of a Hilbert modular form of weight $(1,1)$. The seesaw identity (13.14) for $N=2$ thus relates the two Sczech cocycles introduced separately in $[42,43]$.

13.5. Modular complex and modular units. To conclude, we consider an example related to a regulator map from higher algebraic $K$-theory. Namely, we set $N=2$, so that

$$
S^{+}=\mathrm{GL}_{2}(\mathbf{R})^{+} / \mathrm{SO}_{2} Z(\mathbf{R})^{+} \simeq \mathcal{H} \times \mathbf{R}_{>0} .
$$

Recall from Section $\$ 9$ that we have defined a smooth function

$$
E_{\nu} \in A^{0}(\mathcal{H} \times \mathbf{C})^{\mathrm{SL}_{2}(\mathbf{Z}) \ltimes \mathbf{Z}^{2}},
$$

associated to a function $\nu \in A^{0}(\mathcal{L})$ on the Hodge line bundle $\mathcal{L}$ over $\mathcal{H}$, that is rapidly decreasing along the fibers.

For any integer $m$ we have

$$
E_{\nu}^{(m)}:=E(\tau, m z ; \nu, 0)-m^{2} E(\tau, z ; \nu, 0)=\frac{1}{2 \pi} \log \left|g_{0, m}(\tau, z)\right|,
$$

where $g_{0, m}$ is the modular unit in $\mathcal{O}(Y(\ell))^{\times}$(with $m \mid \ell$ ), defined in (10.2). More generally, one can attach a modular unit $g_{\chi}$ to every Dirichlet character $\chi$.

The map $\log |\cdot|: \mathcal{O}(Y(\ell))^{\times} \rightarrow A^{0}(Y(\ell))$ is an example of a regulator. A more interesting example is the map

$$
\begin{aligned}
\operatorname{reg}: \wedge^{2} \mathcal{O}(Y(\ell))^{\times} & \rightarrow A^{1}(Y(\ell)) \\
u \wedge u^{\prime} & \mapsto i \log \left|u^{\prime}\right| d \arg u-i \log |u| d \arg u^{\prime} .
\end{aligned}
$$

We now briefly explain how to recover this regulator as a theta lift for the dual pair $\left(\mathrm{GL}_{2}, \mathrm{GL}_{2}\right)$. Consider the bundle $\mathcal{L}^{2} \rightarrow \mathcal{H}$. Writing $\pi_{1}, \pi_{2}$ for the canonical projections $\mathcal{L}^{2} \rightarrow \mathcal{L}$, let $\nu_{i}=\pi_{i}^{*} \nu(i=1,2)$ and define

$$
\begin{aligned}
\omega_{1}\left(\nu_{1} \wedge \nu_{2}\right):= & \nu_{1} \partial \nu_{2}-\nu_{2} \partial \nu_{1} \\
& -\nu_{1} \bar{\partial} \nu_{2}+\nu_{2} \bar{\partial} \nu_{1} \in A^{1}\left(\mathcal{L}^{2}\right) .
\end{aligned}
$$

\footnotetext{
${ }^{8}$ This conjecture has been verified in many cases by Colmez [19], and has been announced by Harder [29, 30].
} 
Remark. One motivation to consider this form comes from (9.11) and Goncharov's explicit formulas for regulators (cf. [27, (3.19)]); this form also appears naturally in a formalism of Chern character in equivariant Deligne cohomology.

The underlying real vector bundle of $\mathcal{L}^{2}$ is $E^{2}=E \otimes \mathbf{R}^{2}$ whose fiber is canonically identified with $V^{2}$; in particular we may identify a pair $\left(v_{1}, v_{2}\right) \in V^{2}$ with a section of $\mathcal{L}^{2}$. We obtain a Schwartz form

$$
\tilde{\nu}\left(v_{1}, v_{2}\right):=\left(v_{1}, v_{2}\right)^{*} \omega_{1}\left(\nu_{1} \wedge \nu_{2}\right) \in\left[\mathcal{S}\left(V^{2}\right) \otimes A^{1}(\mathcal{H})\right]^{\mathrm{GL}_{2}(\mathbf{R})} .
$$

Here $\mathrm{GL}_{2}(\mathbf{R})$ acts diagonally on $V^{2}$. Choosing Schwartz functions $\varphi_{f, 1}, \varphi_{f, 2} \in \mathcal{S}\left(V\left(\mathbf{A}_{f}\right)\right)$ fixed by the congruence subgroup $K(\ell) \subset \mathrm{GL}_{2}\left(\mathbf{A}_{f}\right)$, we may form a theta series

$$
\theta\left(g ; \varphi_{f} \otimes \tilde{\nu}\right):=\sum_{v \in V(\mathbf{Q})^{2}}\left(\varphi_{f} \otimes \tilde{\nu}\right)(v \cdot g), \quad\left(\varphi_{f}=\varphi_{f, 1} \otimes \varphi_{f, 2}\right)
$$

that defines a 1-form on $Y(\ell)$ with values in automorphic forms on $\mathrm{GL}_{2}$, or a 'kernel' in $\mathcal{A}\left(\mathrm{GL}_{2}\right) \otimes A^{1}(Y(\ell))$. Consider the seesaw

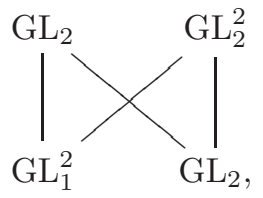

where the vertical lines denote inclusions: $\mathrm{GL}_{1}^{2} \subset \mathrm{GL}_{2}$ gives the torus of diagonal matrices and $\mathrm{GL}_{2} \subset \mathrm{GL}_{2}^{2}$ is the diagonal embedding. If $\chi_{1}, \chi_{2}$ are Dirichlet characters (that we identify with automorphic forms on $\mathrm{GL}_{1}(\mathbf{A})$ ) and we choose $\varphi_{f}$ appropriately, then the seesaw identity shows that

$$
\left\langle\chi_{1} \times \chi_{2}, \theta\left(\varphi_{f} \otimes \tilde{\nu}\right)\right\rangle=\operatorname{reg}\left(g_{\chi_{1}} \wedge g_{\chi_{2}}\right) .
$$

The seesaw can therefore be interpreted as defining a 1-form on $Y(\ell)$ for every pair of cusps in $\mathbf{P}^{1}(\mathbf{Q})$; moreover, this 1-form is the regulator of a product of two modular units, compare with the work of Brunault [13] on the explicit Beilinson-Kato relations.

\section{REFERENCES}

[1] M. F. Atiyah, H. Donnelly, and I. M. Singer. Eta invariants, signature defects of cusps, and values of $L$ functions. Ann. of Math. (2), 118(1):131-177, 1983.

[2] Alexander Beilinson, Guido Kings, and Andrey Levin. Topological polylogarithms and $p$-adic interpolation of $L$-values of totally real fields. Math. Ann., 371(3-4):1449-1495, 2018.

[3] Nicole Berline, Ezra Getzler, and Michèle Vergne. Heat kernels and Dirac operators. Grundlehren Text Editions. Springer-Verlag, Berlin, 2004. Corrected reprint of the 1992 original.

[4] A. Berlinson and A. Levin. The elliptic polylogarithm. In Motives (Seattle, WA, 1991), volume 55 of Proc. Sympos. Pure Math., pages 123-190. Amer. Math. Soc., Providence, RI, 1994.

[5] A. A. Berlinson. Higher regulators and values of $L$-functions. In Current problems in mathematics, Vol. 24, Itogi Nauki i Tekhniki, pages 181-238. Akad. Nauk SSSR, Vsesoyuz. Inst. Nauchn. i Tekhn. Inform., Moscow, 1984.

[6] J.-M. Bismut and J. Cheeger. Remarks on the index theorem for families of Dirac operators on manifolds with boundary. In Differential geometry, volume 52 of Pitman Monogr. Surveys Pure Appl. Math., pages 59-83. Longman Sci. Tech., Harlow, 1991.

[7] J.-M. Bismut, H. Gillet, and C. Soulé. Analytic torsion and holomorphic determinant bundles. I. Bott-Chern forms and analytic torsion. Comm. Math. Phys., 115(1):49-78, 1988.

[8] Jean-Michel Bismut and Jeff Cheeger. Transgressed Euler classes of $\operatorname{SL}(2 n, \mathbf{Z})$ vector bundles, adiabatic limits of eta invariants and special values of $L$-functions. Ann. Sci. École Norm. Sup. (4), 25(4):335-391, 1992.

[9] Don Blasius. On the critical values of Hecke L-series. Ann. of Math. (2), 124(1):23-63, 1986. 
[10] Lev A. Borisov and Paul E. Gunnells. Toric modular forms and nonvanishing of $L$-functions. J. Reine Angew. Math., 539:149-165, 2001.

[11] Lev A. Borisov and Paul E. Gunnells. Toric varieties and modular forms. Invent. Math., 144(2):297-325, 2001.

[12] Raoul Bott and Loring W. Tu. Differential forms in algebraic topology, volume 82 of Graduate Texts in Mathematics. Springer-Verlag, New York-Berlin, 1982.

[13] François Brunault. Beilinson-Kato elements in $K_{2}$ of modular curves. Acta Arith., 134(3):283-298, 2008.

[14] Pierrette Cassou-Noguès. Valeurs aux entiers négatifs des fonctions zêta et fonctions zêta $p$-adiques. Invent. Math., 51(1):29-59, 1979.

[15] Pierre Charollois and Samit Dasgupta. Integral Eisenstein cocycles on $\mathbf{G L}_{n}$, I: Sczech's cocycle and $p$-adic $L$-functions of totally real fields. Camb. J. Math., 2(1):49-90, 2014.

[16] Pierre Charollois, Samit Dasgupta, and Matthew Greenberg. Integral Eisenstein cocycles on $\mathbf{G L}_{n}$, II: Shintani's method. Comment. Math. Helv., 90(2):435-477, 2015.

[17] Pierre Charollois and Robert Sczech. Elliptic functions according to Eisenstein and Kronecker: an update. Eur. Math. Soc. Newsl., (101):8-14, 2016.

[18] Shiing-shen Chern. A simple intrinsic proof of the Gauss-Bonnet formula for closed Riemannian manifolds. Ann. of Math. (2), 45:747-752, 1944.

[19] P. Colmez. Algébricité de valeurs spéciales de fonctions L. Invent. Math., 95(1):161-205, 1989.

[20] R. M. Damerell. $L$-functions of elliptic curves with complex multiplication. I. Acta Arith., 17:287-301, 1970.

[21] P. Deligne. Valeurs de fonctions $L$ et périodes d'intégrales. In Automorphic forms, representations and $L$ functions (Proc. Sympos. Pure Math., Oregon State Univ., Corvallis, Ore., 1977), Part 2, Proc. Sympos. Pure Math., XXXIII, pages 313-346. Amer. Math. Soc., Providence, R.I., 1979. With an appendix by N. Koblitz and A. Ogus.

[22] Pierre Deligne and Kenneth A. Ribet. Values of abelian $L$-functions at negative integers over totally real fields. Invent. Math., 59(3):227-286, 1980.

[23] Gerd Faltings. Arithmetic Eisenstein classes on the Siegel space: some computations. In Number fields and function fields-two parallel worlds, volume 239 of Progr. Math., pages 133-166. Birkhäuser Boston, Boston, MA, 2005.

[24] Jorge Flórez, Cihan Karabulut, and Tian An Wong. Eisenstein cocycles over imaginary quadratic fields and special values of L-functions. J. Number Theory, 204:497-531, 2019.

[25] Luis E. Garcia. Superconnections, theta series, and period domains. Adv. Math., 329:555-589, 2018.

[26] Ezra Getzler. The Thom class of Mathai and Quillen and probability theory. In Stochastic analysis and applications (Lisbon, 1989), volume 26 of Progr. Probab., pages 111-122. Birkhäuser Boston, Boston, MA, 1991.

[27] Alexander B. Goncharov. Regulators. In Handbook of $K$-theory. Vol. 1, 2, pages 295-349. Springer, Berlin, 2005.

[28] P. Graf. Polylogarithms for GL 2 over totally real fields. ArXiv e-prints, April 2016.

[29] G. Harder and N. Schappacher. Special values of Hecke $L$-functions and abelian integrals. In Workshop Bonn 1984 (Bonn, 1984), volume 1111 of Lecture Notes in Math., pages 17-49. Springer, Berlin, 1985.

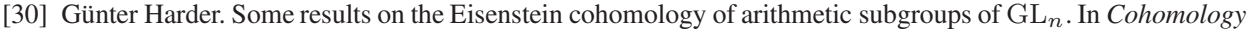
of arithmetic groups and automorphic forms (Luminy-Marseille, 1989), volume 1447 of Lecture Notes in Math., pages 85-153. Springer, Berlin, 1990.

[31] G. H. Hardy and E. M. Wright. An introduction to the theory of numbers. Oxford University Press, Oxford, sixth edition, 2008. Revised by D. R. Heath-Brown and J. H. Silverman, With a foreword by Andrew Wiles.

[32] R. Howe. $\theta$-series and invariant theory. In Automorphic forms, representations and L-functions (Proc. Sympos. Pure Math., Oregon State Univ., Corvallis, Ore., 1977), Part 1, Proc. Sympos. Pure Math., XXXIII, pages 275-285. Amer. Math. Soc., Providence, R.I., 1979.

[33] Taku Ishii and Takayuki Oda. A short history on investigation of the special values of zeta and $L$-functions of totally real number fields. In Automorphic forms and zeta functions, pages 198-233. World Sci. Publ., Hackensack, NJ, 2006.

[34] Hiroshi Ito. A function on the upper half space which is analogous to the imaginary part of $\log \eta(z) . J$. Reine Angew. Math., 373:148-165, 1987.

[35] Kazuya Kato. $p$-adic Hodge theory and values of zeta functions of modular forms. Number 295, pages ix, 117-290. 2004. Cohomologies $p$-adiques et applications arithmétiques. III.

[36] Helmut Klingen. über die Werte der Dedekindschen Zetafunktion. Math. Ann., 145:265-272, 1961/1962.

[37] Stephen S. Kudla. Seesaw dual reductive pairs. In Automorphic forms of several variables (Katata, 1983), volume 46 of Progr. Math., pages 244-268. Birkhäuser Boston, Boston, MA, 1984. 
[38] Stephen S. Kudla and John J. Millson. Intersection numbers of cycles on locally symmetric spaces and Fourier coefficients of holomorphic modular forms in several complex variables. Inst. Hautes Études Sci. Publ. Math., (71):121-172, 1990.

[39] Varghese Mathai and Daniel Quillen. Superconnections, Thom classes, and equivariant differential forms. Topology, 25(1):85-110, 1986.

[40] Werner Müller. Signature defects of cusps of Hilbert modular varieties and values of $L$-series at $s=1 . J$. Differential Geom., 20(1):55-119, 1984.

[41] Madhav V. Nori. Some Eisenstein cohomology classes for the integral unimodular group. In Proceedings of the International Congress of Mathematicians, Vol. 1, 2 (Zürich, 1994), pages 690-696. Birkhäuser, Basel, 1995.

[42] Robert Sczech. Dedekindsummen mit elliptischen Funktionen. Invent. Math., 76(3):523-551, 1984.

[43] Robert Sczech. Eisenstein cocycles for $\mathrm{GL}_{2} \mathbf{Q}$ and values of $L$-functions in real quadratic fields. Comment. Math. Helv., 67(3):363-382, 1992.

[44] Robert Sczech. Eisenstein group cocycles for $\mathrm{GL}_{n}$ and values of $L$-functions. Invent. Math., 113(3):581616, 1993.

[45] Takuro Shintani. On evaluation of zeta functions of totally real algebraic number fields at non-positive integers. J. Fac. Sci. Univ. Tokyo Sect. IA Math., 23(2):393-417, 1976.

[46] Carl Ludwig Siegel. Über die analytische Theorie der quadratischen Formen. III. Ann. of Math. (2), 38(1):212-291, 1937.

[47] Carl Ludwig Siegel. Advanced analytic number theory, volume 9 of Tata Institute of Fundamental Research Studies in Mathematics. Tata Institute of Fundamental Research, Bombay, second edition, 1980.

[48] Carl Ludwig Siegel. Gesammelte Abhandlungen. IV. Springer Collected Works in Mathematics. Springer, Heidelberg, 2015. Edited by Komaravolu Chandrasekharan and Hans Maaß, Reprint of the 1979 edition [ MR0543842], Original publication incorrectly given as 1966 edition on the title page.

[49] Dennis Sullivan. La classe d'Euler réelle d'un fibré vectoriel à groupe structural $\mathrm{SL}_{n}(Z)$ est nulle. $C . R$. Acad. Sci. Paris Sér. A-B, 281(1):Aii, A17-A18, 1975.

[50] André Weil. Elliptic functions according to Eisenstein and Kronecker. Classics in Mathematics. SpringerVerlag, Berlin, 1999. Reprint of the 1976 original.

[51] Franck Wielonsky. Séries d'Eisenstein, intégrales toroïdales et une formule de Hecke. Enseign. Math. (2), 31(1-2):93-135, 1985.

ENS / PSl University, Département de Mathématiques et Applications, F-75005, Paris, FRANCE

E-mail address: nicolas.bergeroneens.fr

URL: https://sites.google.com/view/nicolasbergeron/accueil

Sorbonne Université, Institut de Mathématiques de Jussieu-Paris Rive Gauche, CNRS, UNIV PARIS Diderot, F-75005, PARIS, FrancE

E-mail address: pierre.charollois@imj-prg.fr

URL: http://people.math.jussieu.fr/ charollois

Department of Mathematics, University College London, Gower Street, London WC1E 6BT, UNITED KINGDOM

E-mail address: l.e.garcia@ucl.ac.uk 